\title{
Analyzing high-energy factorization beyond next-to-leading logarithmic accuracy
}

\author{
Vittorio Del Duca, ${ }^{a}$ Giulio Falcioni, ${ }^{b}$ Lorenzo Magnea $^{b}$ and Leonardo Vernazza ${ }^{b}$ \\ ${ }^{a}$ INFN, Laboratori Nazionali Frascati, \\ 00044 Frascati (Roma), Italy \\ ${ }^{b}$ Dipartimento di Fisica Teorica, Università di Torino and \\ INFN, Sezione di Torino, \\ Via P. Giuria 1, I-10125 Torino, Italy \\ E-mail: delduca@lnf.infn.it, giulio.falcioni@unito.it, \\ lorenzo.magnea@unito.it, vernazza@to.infn.it
}

ABSTRACT: We provide a complete and detailed study of the high-energy limit of fourparton scattering amplitudes in QCD, giving explicit results at two loops and higher orders, and going beyond next-to-leading logarithmic (NLL) accuracy. Building upon recent results, we use the techniques of infrared factorization to investigate the failure of the simplest form of Regge factorization, starting at next-to-next-to-leading logarithmic accuracy (NNLL) in $\ln (s /|t|)$. We provide detailed accounts and explicit expressions for the terms responsible for this breaking in the case of two-loop and three-loop quark and gluon amplitudes in QCD; in particular, we recover and explain a known non-logarithmic double-pole contribution at two-loops, and we compute all non-factorizing single-logarithmic singular contributions at three loops. Conversely, we use high-energy factorization to show that the hard functions of infrared factorization vanish in $d=4$ to all orders in the coupling, up to NLL accuracy in $\ln (s /|t|)$. This provides clear evidence for the infrared origin of high-energy logarithms. Finally, we extend earlier studies to $t$-channel exchanges of color representations beyond the octet, which enables us to give predictions based on the dipole formula for single-pole NLL contributions at three and four loops.

KeYwords: QCD Phenomenology, Hadronic Colliders

ARXIV EPRINT: 1409.8330 


\section{Contents}

1 Introduction 1

2 Infrared factorization and the high-energy limit 5

3 High-energy color structure and the signature of the amplitude $\quad 8$

4 Comparing infrared and high-energy factorizations for octet exchange 11

$\begin{array}{lll}4.1 & \text { One-loop matrix elements } & 13\end{array}$

$\begin{array}{lll}4.2 & \text { Two-loop matrix elements } & 15\end{array}$

$\begin{array}{lll}4.3 & \text { Three-loop matrix elements } & 21\end{array}$

$\begin{array}{ll}4.4 & \text { Beyond three loops }\end{array}$

$\begin{array}{llr}5 & \text { On subleading color amplitudes } & 29\end{array}$

6 Conclusion 33

A A color basis for four-parton amplitudes in QCD 35

$\begin{array}{lll}\text { A.1 Quark-quark scattering } & 35\end{array}$

$\begin{array}{lll}\text { A.2 Gluon-gluon scattering } & 35\end{array}$

$\begin{array}{lll}\text { A.3 Quark-gluon scattering } & 37\end{array}$

$\begin{array}{ll}\text { B Anomalous dimensions } & 37\end{array}$

C Hard functions for four-parton amplitudes in the high-energy limit $\quad 39$

$\begin{array}{lll}\text { C.1 Quark-quark scattering } & 40\end{array}$

$\begin{array}{lll}\text { C.2 Gluon-gluon scattering } & 41\end{array}$

C.3 Quark-gluon scattering 43

$\begin{array}{ll}\text { D Infrared singularities for singlet exchange } & 44\end{array}$

\section{Introduction}

In the high-energy limit, in which the squared centre-of-mass energy $s$ is much larger than the typical momentum transfer $(-t)$, so that $|s / t| \rightarrow \infty$ with $t$ held fixed, a four-point treelevel gauge theory scattering amplitude acquires a factorized structure, given by a $t$-channel propagator, associated with the highest-spin particle in the theory (in the case of QCD, a gluon), connecting two emission vertices, termed impact factors, which characterize the particles undergoing the scattering. The impact factors depend on the specific scattering process, while the $t$-channel propagator is process independent. 
When loop corrections are included, the $t$-channel propagator gets dressed according to the schematic form [1],

$$
\frac{1}{t} \rightarrow \frac{1}{t}\left(\frac{s}{-t}\right)^{\alpha(t)}
$$

where $\alpha(t)$ is a function of the coupling constant and of the momentum transfer $t$, which can be expanded perturbatively at weak coupling. The expansion of eq. (1.1) in powers of the coupling then generates the leading logarithmic corrections to the amplitude in $\ln (s /|t|)$. Because of the analytic structure of eq. (1.1), which is typical of Regge theory, $\alpha(t)$ is called Regge trajectory.

For the real part of the amplitude, the t-channel picture, often termed high-energy factorization, is in fact accurate at leading and at next-to-leading logarithmic (NLL) accuracy in $\ln (s /|t|)[2]$. Because the amplitude has a $t$-channel ladder-like structure, we can assume it to be even under $s \leftrightarrow u$ exchange. As a consequence, it must be composed of kinematic and color parts which are either both even or both odd under $s \leftrightarrow u$ exchange, a feature commonly referred to as 'signature' of the amplitude in the literature on Regge theory. As an example, let us consider the amplitude for gluon-gluon scattering. In this case, for the process $g\left(k_{1}\right)+g\left(k_{2}\right) \rightarrow g\left(k_{3}\right)+g\left(k_{4}\right)$, one may write [3]

$$
\begin{gathered}
\mathcal{M}_{a_{1} a_{2} a_{3} a_{4}}^{g g g g}\left(\frac{s}{\mu^{2}}, \frac{t}{\mu^{2}}, \alpha_{s}\left(\mu^{2}\right)\right)=4 \pi \alpha_{s}\left(\mu^{2}\right) \frac{s}{t}\left[\left(\mathbf{T}^{b}\right)_{a_{1} a_{3}} C_{\lambda_{1} \lambda_{3}}\left(k_{1}, k_{3}\right)\right] \\
\times\left[\left(\frac{s}{-t}\right)^{\alpha(t)}+\left(\frac{-s}{-t}\right)^{\alpha(t)}\right]\left[\left(\mathbf{T}_{b}\right)_{a_{2} a_{4}} C_{\lambda_{2} \lambda_{4}}\left(k_{2}, k_{4}\right)\right]
\end{gathered}
$$

where $a_{j}$ and $k_{j}$ are the color index and momentum of gluon $j$, and $\mathbf{T}^{b}$ is a color generator in the adjoint representation, so that $\left(\mathbf{T}^{a}\right)_{b c}=-\mathrm{i} f_{a b c}$. The impact factors, $C_{\lambda_{i} \lambda_{j}}\left(k_{i}, k_{j}\right)$, depend on the helicities of the gluons, but are independent of the squared centre-of-mass energy $s$. In the weak coupling limit, both the impact factors and the Regge trajectory can be expanded in powers of the renormalized coupling $\alpha_{s}\left(\mu^{2}\right)$ : they are then affected by infrared and collinear divergences, which are (implicitly) regularized by dimensional regularization in eq. (1.2).

Beyond leading order, and for the real part of the amplitude beyond the NLL accuracy in $\ln (s /|t|)$, one should consider also the exchange of two or more reggeized gluons. Accordingly, one should include the contribution to the amplitude in which the kinematic and color parts are both even under $s \leftrightarrow u$ exchange, and in particular the case in which a color singlet is exchanged.

The process independence of $t$-channel gluon exchange implies that one can write formulae similar to eq. (1.2) for quark-quark and quark-gluon scattering, differing only for the presence of the quark impact factor instead of the gluon impact factor. Considering them together with gluon-gluon scattering, as given by eq. (1.2), one obtains a system of three equations, which can be used to determine the impact factors for quark and gluon scattering. In fact, one gets an over-constrained system of three equations and two unknowns. One can use two of the equations to determine the one-loop impact factors, and the third to perform a consistency check on high-energy factorization. Because the 
Regge trajectory and the impact factors can be expanded as a series in the coupling, this procedure can be repeated in principle at each loop order, although it is unwarranted for terms beyond the NLL accuracy. Specifically, the expansion of the Regge trajectory and of the impact factors at one loop shows, as expected, that each equation has a term proportional to $\ln (s /|t|)$, which is the same for all three amplitudes. That term gives the one-loop Regge trajectory, and the fact that it is the same for all three equations shows its universality, i.e. its independence of the particular scattering process under consideration. Conversely, the term independent of $\ln (s /|t|)$ is different for each equation. One can then use two of the equations to determine the one-loop gluon and quark impact factors, and use the third to check the consistency of high-energy factorization.

Repeating the procedure above at two loops, one can use the terms proportional to $\ln (s /|t|)$ to determine the two-loop Regge trajectory and verify its universality, and the terms independent of $\ln (s /|t|)$ to compute the two-loop impact factors and check that high-energy factorization holds. Such a check, however, fails [4], due to the presence of a term proportional to $\alpha_{s}^{2} \pi^{2} / \epsilon^{2}$, which therefore invalidates high-energy factorization, making the determination of the two-loop impact factors ambiguous. This is not totally unexpected, since terms independent of $\ln (s /|t|)$ at two loops are beyond NLL accuracy. It is however interesting to notice that the violation originates only in the term proportional to $\pi^{2} / \epsilon^{2}$, and not in the single pole nor in the finite part of the would-be impact factors. Furthermore, it must be emphasized that, in the context of Regge theory, eq. (1.2) is only an approximation, based on the assumption that only Regge poles appear in the angular momentum plane. Regge theory arguments predating QCD (see, for example, ref. [5]) suggest that this approximation is likely to break down, for logarithmic terms, at the three-loop level, at NNLL accuracy and for non-planar contributions to the amplitude. At this accuracy, one may in fact envisage contributions to the amplitude due to Regge cuts in the angular momentum plane, which are absent in expressions such as eq. (1.2). These corrections to Regge-pole-based high-energy factorization were never previously pinned down in any specific computation of a scattering amplitude in the high-energy limit. The violation of universality observed at two loops in ref. [4], as we show in the present paper, is a harbinger of precisely such phenomena at the three-loop level.

In recent years, a general approach to the high-energy limit of scattering amplitudes based on the universal properties of their infrared singularities has been developed in $[6,7]$, following the earlier results of [8-10]. This approach suggests, in particular, that the violation of high-energy factorization reported in [4] at order $\alpha_{s}^{2}$ and at next-to-next-to-leading logarithmic accuracy in $\ln (s /|t|)$ is due to the amplitude becoming non-diagonal in the $t$ channel-exchange basis. Such a violation iterates then at three loops in the $\alpha_{s}^{3}$ term proportional to $\ln (s /|t|)$, invalidating the universality of the three-loop Regge trajectory. Thus, the eventual definition of a universal three-loop Regge trajectory requires additional conditions.

In refs. $[11,12]$ we have further developed the approach above, identifying the origin of the high-energy factorization violation discovered in [4] at two loops. In order to be able to define unambiguously a universal Regge trajectory and the related impact factors beyond the NLL accuracy, we have proposed a way to isolate factorization-breaking terms at three loops and beyond. This goal can be achieved introducing a non-factorizing contribution 
to the amplitude, whose infrared and collinear divergent parts can then be unambiguously predicted using the tools described in $[6,7]$. We believe that a framework for consistently identifying factorizing and non-factorizing contributions to high-energy amplitudes can be useful both in practical finite-order calculations, to assess the reliability of high-energy resummations, and for theoretical developments. Indeed, a precise expression for the discrepancy between pole-based Regge factorization and the actual perturbative results for the amplitude may be useful at least as a boundary condition for future attempts to extend high-energy factorization to include the contributions of Regge cuts. Furthermore, our results are a first step in the direction of systematically combining information on amplitudes which arise from infrared factorization, which is exact to all orders in perturbation theory for all singular contributions to the amplitudes, with those arising from Regge factorization, which applies also to finite contributions to the amplitudes, but has limited validity in terms of logarithmic accuracy.

In this paper, we extend the analysis of refs. [11, 12] beyond leading poles, beyond three loops and beyond the leading color amplitude. Furthermore, we provide a more flexible framework for combining infrared factorization with the high-energy limit, which is better suited to disentangle the various color components of the amplitudes. We are then able to provide detailed predictions for singular terms contributing to the high-energy limit of quark and gluon amplitudes in QCD up to three loops, and furthermore we are able to derive towers of constraints on real and imaginary parts of finite contributions to the amplitudes, valid to all orders in perturbation theory, up to NLL accuracy. These constraints show that, to the stated accuracy, the hard (infrared-finite) parts of the amplitudes can be chosen to vanish, so that all high energy logarithms (up to NLL included) are generated by the infrared operators arising from infrared factorization. This result lends support to the conjecture that all high-energy logarithms may be understood as originating from a special class of infrared enhancements, as suggested in [8-10, 13-15], and also in agreement with the recently proposed approach of ref. [16].

The paper is organized as follows. In section 2, we discuss the general features of infrared factorization, and we review the results of ref. [6]. In section 3, we provide a general parametrization of four-point scattering amplitudes in the high-energy limit, and we introduce an improved organization of infrared operators, which better adapts to the color structure and symmetry properties of scattering amplitude in the high-energy limit. In section 4 we give a detailed comparison of infrared and high-energy factorizations up to three-loop order. This allows us to recover the results of refs. [4, 11, 12], and to provide definite and complete predictions for factorization-breaking terms at three loops. Furthermore, we examine the coefficients of the hard functions in the high-energy limit to all orders in the coupling, up to NLL accuracy in $\ln (s /|t|)$. In section 5 , we analyze the $t$-channel exchange of color representations other than the octet, which do not admit a high-energy factorization as in eq. (1.2), and we provide a comparison, based on the dipole formula for the soft anomalous dimension matrix, to similar studies performed in terms of Wilson lines in ref. [16]. In section 6 , we briefly discuss our results and the prospects for future developments. Finally, several technical details which, we believe, will be useful for future high-order calculations of high-energy quark and gluon amplitudes, are given in the 
appendices. In appendix A, we provide the color bases we use for four-point scattering amplitudes, in appendix B we give the expressions for all relevant anomalous dimensions up to three loops, while in appendix $\mathrm{C}$ we write down the high-energy limit of the hard functions of four-point QCD scattering amplitudes, up to two-loop accuracy, using the exact four-point two-loop amplitudes provided in the literature [17-21]. In addition, as an example, in appendix D we provide expressions for the singularities of singlet exchange amplitudes up to two-loop accuracy.

\section{Infrared factorization and the high-energy limit}

Matrix elements for quark and gluon scattering have a non-trivial color structure, which is best understood by assembling them into vectors in the space of color configurations available for the process at hand. In general one writes, for $n$-parton scattering,

$$
\mathcal{M}_{n}\left(\frac{p_{i}}{\mu}, \alpha_{s}\left(\mu^{2}\right)\right)=\sum_{j} \mathcal{M}_{n}^{[j]}\left(\frac{p_{i}}{\mu}, \alpha_{s}\left(\mu^{2}\right)\right) c_{[j]}^{(n)},
$$

where the $c_{[j]}^{(n)}$ 's are color tensors of rank $n$, with indices (not shown here) in the color representations of the external partons undergoing the scattering, while the index $[j]=$ $1, \ldots, r$ enumerates the color representations which can be exchanged in intermediate states in a selected channel. For a detailed discussion of how such tensors can be enumerated and constructed, when the external particles are in arbitrary color representations, we refer the reader to [6, 22-24]: briefly, having selected for example an $s$-channel basis, one must construct the tensor product of the initial state representations, and take its intersection with the tensor product of final state representations. We note that, as in the rest of the paper, in eq. (2.1) we left implicit the dependence on the infrared regulator $\epsilon=2-d / 2<0$.

Infrared and collinear singularities in the vector $\mathcal{M}_{n}$ are known to factorize, ${ }^{1}$ so that the matrix element can be written as

$$
\mathcal{M}_{n}\left(\frac{p_{i}}{\mu}, \alpha_{s}\left(\mu^{2}\right)\right)=\mathcal{Z}_{n}\left(\frac{p_{i}}{\mu}, \alpha_{s}\left(\mu^{2}\right)\right) \mathcal{H}_{n}\left(\frac{p_{i}}{\mu}, \alpha_{s}\left(\mu^{2}\right)\right) .
$$

Here $\mathcal{H}$ is a color vector, which is finite as $\epsilon \rightarrow 0$, and represents a matching condition, to be determined order by order in perturbation theory after the subtraction of divergent contributions. The infrared operator $\mathcal{Z}_{n}$, on the other hand, is an $r \times r$ matrix in color space, generating all infrared and collinear singularities of the amplitude; it satisfies a (matrix) renormalization group equation, whose general solution can be written in the form

$$
\mathcal{Z}_{n}\left(\frac{p_{i}}{\mu}, \alpha_{s}\left(\mu^{2}\right)\right)=\mathcal{P} \exp \left[\frac{1}{2} \int_{0}^{\mu^{2}} \frac{d \lambda^{2}}{\lambda^{2}} \Gamma_{n}\left(\frac{p_{i}}{\lambda}, \alpha_{s}\left(\lambda^{2}\right)\right)\right],
$$

where $\mathcal{P}$ denotes path ordering in color space. Note that all poles in $\epsilon$ are generated [29] through the integration of the $d$-dimensional running coupling down to vanishing scale,

\footnotetext{
${ }^{1}$ See $[25-27]$ and references therein for discussions of matrix element factorization. An analysis in the context of SCET was recently proposed in ref. [28].
} 
$\lambda \rightarrow 0$. For massless particles, the results of refs. [30, 31] showed that, up to two loops, the $n$-parton soft anomalous dimension matrix has a remarkably simple form, proportional to the one-loop result, regardless of the number of partons involved. This stimulated further investigations, and led to the proposal of the 'dipole formula' [32-35] as an all-order ansatz for $\Gamma_{n}$. This takes the form

$$
\Gamma_{n}^{\operatorname{dip}}\left(\frac{p_{i}}{\lambda}, \alpha_{s}\left(\lambda^{2}\right)\right)=\frac{1}{4} \widehat{\gamma}_{K}\left(\alpha_{s}\left(\lambda^{2}\right)\right) \sum_{(i, j)} \ln \left(\frac{-s_{i j}}{\lambda^{2}}\right) \mathbf{T}_{i} \cdot \mathbf{T}_{j}-\sum_{i=1}^{n} \gamma_{i}\left(\alpha_{s}\left(\lambda^{2}\right)\right)
$$

The basic feature of eq. (2.4) is that the color structure, expressed in terms of the colorinsertion operator $\mathbf{T}^{i}$ for parton $i$, remains the same as at leading order, and therefore it is expressed as a sum over color dipoles, with all higher-order multipoles vanishing exactly. Color and kinematics are tightly correlated, since momentum dependence occurs only through the 'dipole' invariants $s_{i j}=\left(p_{i}+p_{j}\right)^{2}$, where for the sake of simplicity we have taken all momenta as outgoing. An important consequence of the simple color structure of eq. (2.4) is that the path ordering symbol in eq. (2.3) can be dropped, since scale dependence through the coupling is confined to colorless anomalous dimensions. These are defined as follows. Denoting by $\gamma_{K}^{[i]}$ the cusp anomalous dimension $[36,37]$ in representation $[i]$, and by $\mathcal{C}_{[i]}$ the corresponding quadratic Casimir eigenvalue, we assume $\gamma_{K}^{[i]}$ to be proportional to $\mathcal{C}_{[i]}$ through a universal function $\hat{\gamma}_{K}$, so that $\gamma_{K}^{[i]}=\mathcal{C}_{[i]} \hat{\gamma}_{K}$. This is known to be true at least up to three loops. The functions $\gamma_{i}$, on the other hand, are collinear anomalous dimensions which can be extracted from form factor data [27, 29, 34].

The dipole formula, eq. (2.4), arises as the simplest solution to a set of exact equations satisfied by the soft anomalous dimension, which can be understood as anomaly equations. Indeed, correlators of semi-infinite straight Wilson lines have a classical symmetry under independent rescalings of all four-velocities $\beta_{i}$, which is broken by quantum corrections for light-like lines, as a consequence of collinear divergences. The kinematic dependence of the soft anomalous dimension in this case is constrained by the cancellation of this anomaly in physical matrix elements. Eq. (2.4) is exact up to two loops for massless partons, as first shown in refs. [30, 31]. The advantage of having exact equations for $\Gamma_{n}$ is that one can study possible corrections to eq. (2.4) in a systematic way [34, 38, 39]. One finds that possible corrections could come only from two sources. They can take the form of conformal cross-ratios of kinematic invariants, starting at three loops and with at least four hard partons, which however are very tightly constrained by symmetry requirements and by known properties of scattering amplitudes, including their high-energy behavior. Alternatively, they can arise as a consequence of violations of Casimir scaling for the cusp anomalous dimension, which can happen in principle starting at four loops. The complete calculation of the three-loop soft anomalous dimension matrix $\Gamma_{n}$ is a very challenging project, and recent progress to this end has been discussed in [40, 41]. Also recently, evidence for the existence of corrections to eq. (2.4) at the four-loop level, and at NLL accuracy in the high-energy limit, was uncovered in ref. [16]. Finally, the results of the recent calculation of the three-loop non-light-like cusp anomalous dimension in ref. [42] led to conjecture a possible violation of the Casimir scaling of $\gamma_{K}\left(\alpha_{s}\right)$, at four loops and for contributions proportional to $n_{f}$. 
For the rest of this paper, we will simply assume that the dipole formula is correct. Essentially all of the results given below are in any case not affected by possible corrections. When this is not the case, for example for single pole terms at three loops, we will explicitly note how the results could change. In the remainder of this section, we discuss the implications of the dipole formula for the high-energy limit, specializing to four-point amplitudes, which are the simplest and most studied case. Here we summarize the results of refs. $[6,7,11,12]$, while in section 3 we propose an improved organization of infrared factors, which yields a more transparent comparison with high-energy factorization.

The main result of refs. $[6,7,11,12]$ is that, in the high energy limit, the infrared factor $\mathcal{Z}_{n}$ takes a simple factorized form, which is valid to leading power in $|t| / s$ and to all logarithmic accuracies. In the case of four-point amplitudes, one can write

$$
\mathcal{Z}\left(\frac{s}{\mu^{2}}, \frac{t}{\mu^{2}}, \alpha_{s}\right)=\exp \left[-\mathrm{i} \frac{\pi}{2} K\left(\alpha_{s}\right) \mathcal{C}_{\text {tot }}\right] \mathcal{Z}_{1, \mathbf{R}}\left(\frac{t}{\mu^{2}}, \alpha_{s}\right) \widetilde{\mathcal{Z}}\left(\frac{s}{t}, \alpha_{s}\right)+\mathcal{O}\left(\frac{t}{s}\right),
$$

where, for simplicity, we omit henceforth the label $n=4$, and where $\mathcal{C}_{\text {tot }} \equiv \sum_{i=1}^{4} \mathcal{C}_{[i]}$ is the sum of the Casimir eigenvalues of the external particles. The main ingredient of eq. (2.5) is the matrix $\widetilde{\mathcal{Z}}$, which encodes the dependence on high-energy logarithms, and carries non-trivial color information, which will be crucial for our discussion. It is given by

$$
\widetilde{\mathcal{Z}}\left(\frac{s}{t}, \alpha_{s}\right)=\exp \left\{K\left(\alpha_{s}\right)\left[\log \left(\frac{s}{-t}\right) \mathbf{T}_{t}^{2}+i \pi \mathbf{T}_{s}^{2}\right]\right\} .
$$

$\widetilde{\mathcal{Z}}$ is responsible for generating all high-energy logarithms of the amplitude which are accompanied by infrared poles. In eq. (2.6) we also introduced 'Mandelstam' combinations of color-insertion operators $\mathbf{T}_{s}=\mathbf{T}_{1}+\mathbf{T}_{2}$ and $\mathbf{T}_{t}=\mathbf{T}_{1}+\mathbf{T}_{3}$. The coefficients of the high-energy logarithms are determined by the function

$$
K\left(\alpha_{s}\right)=-\frac{1}{4} \int_{0}^{\mu^{2}} \frac{d \lambda^{2}}{\lambda^{2}} \hat{\gamma}_{K}\left(\alpha_{s}\left(\lambda^{2}\right)\right),
$$

which is a scale integral over the cusp anomalous dimension. This integral is well known in perturbative QCD: it enters the resummation of infrared poles in the quark form factor [29] and in planar multi-parton scattering amplitudes [43]; it was recursively computed to all orders, in terms of the perturbative coefficients of $\beta\left(\alpha_{s}\right)$ and $\gamma_{K}\left(\alpha_{s}\right)$, in [44]; in the context of the high-energy limit, a slightly different form of eq. (2.7) was shown to give the allorder infrared part of the Regge trajectory in [10]. In eq. (2.7) the singular $\epsilon$ dependence is generated through the integration of the $d$-dimensional version of the running coupling, so that the result is a pure counterterm. To three-loop order one finds ${ }^{2}$

$$
K\left(\alpha_{s}\right)=\frac{\alpha_{s}}{\pi} \frac{\widehat{\gamma}_{K}^{(1)}}{4 \epsilon}+\left(\frac{\alpha_{s}}{\pi}\right)^{2}\left(\frac{\widehat{\gamma}_{K}^{(2)}}{8 \epsilon}-\frac{b_{0} \widehat{\gamma}_{K}^{(1)}}{32 \epsilon^{2}}\right)
$$

\footnotetext{
${ }^{2}$ We choose to expand all functions in powers of $\alpha_{s} / \pi$. The explicit expressions for the perturbative coefficients of the various anomalous dimensions we use, up to three loops, are given in appendix B. Note that normalizations must be changed appropriately when comparing with the literature, for example [4], where perturbative expansions are often in powers of $\alpha_{s} /(4 \pi)$.
} 


$$
+\left(\frac{\alpha_{s}}{\pi}\right)^{3}\left(\frac{\widehat{\gamma}_{K}^{(3)}}{12 \epsilon}-\frac{b_{0} \widehat{\gamma}_{K}^{(2)}+b_{1} \widehat{\gamma}_{K}^{(1)}}{48 \epsilon^{2}}+\frac{b_{0}^{2} \widehat{\gamma}_{K}^{(1)}}{192 \epsilon^{3}}\right)+\mathcal{O}\left(\alpha_{s}^{4}\right),
$$

The final ingredient of eq. $(2.5)$ is the function $\mathcal{Z}_{1, \mathbf{R}}$, which is a singlet in color space and real in the physical region. Its explicit expression is

$$
\mathcal{Z}_{1, \mathbf{R}}\left(\frac{t}{\mu^{2}}, \alpha_{s}\right)=\exp \left\{\frac{1}{2}\left[K\left(\alpha_{s}\right) \log \left(\frac{-t}{\mu^{2}}\right)+D\left(\alpha_{s}\right)\right] \mathcal{C}_{\text {tot }}+\sum_{i=1}^{4} B_{i}\left(\alpha_{s}\right)\right\},
$$

where the functions $D\left(\alpha_{s}\right)$ and $B\left(\alpha_{s}\right)$, just like $K\left(\alpha_{s}\right)$, are given by scale integrals of the cusp and collinear anomalous dimensions, and they similarly yield a perturbative series of pure counterterms, representing infrared and collinear divergences. Explicitly,

$$
\begin{aligned}
D\left(\alpha_{s}\right) & =-\frac{1}{4} \int_{0}^{\mu^{2}} \frac{d \lambda^{2}}{\lambda^{2}} \widehat{\gamma}_{K}\left(\alpha_{s}\left(\lambda^{2}\right)\right) \log \left(\frac{\mu^{2}}{\lambda^{2}}\right), \\
B_{i}\left(\alpha_{s}\right) & =-\frac{1}{2} \int_{0}^{\mu^{2}} \frac{d \lambda^{2}}{\lambda^{2}} \gamma_{i}\left(\alpha_{s}\left(\lambda^{2}\right)\right) .
\end{aligned}
$$

Because of the extra logarithm, the function $D\left(\alpha_{s}\right)$ is responsible for double poles combining infrared and collinear singularities. An important property of the operator $\mathcal{Z}_{1, \mathbf{R}}$, relevant for high-energy factorization and manifest in eq. (2.9), is that it can be written to all orders in perturbation theory as the product of four factors, each one associated with one of the external hard partons. Labeling the partons involved in the $2 \rightarrow 2$ scattering process by means of indices $\{\mathrm{r}, \mathrm{s}\}$, with $\{\mathrm{r}, \mathrm{s}\}=\{q, g\}$ for quarks and gluons respectively, so that $\mathcal{Z}_{1, \mathbf{R}} \rightarrow \mathcal{Z}_{1, \mathbf{R}}^{\text {rs }}$, one may write

$$
\mathcal{Z}_{1, \mathbf{R}}^{\mathrm{rs}}\left(\frac{t}{\mu^{2}}, \alpha_{s}\right)=\left(\mathcal{Z}_{1, \mathbf{R}}^{\mathrm{r}}\left(\frac{t}{\mu^{2}}, \alpha_{s}\right)\right)^{2}\left(\mathcal{Z}_{\mathbf{1}, \mathbf{R}}^{\mathrm{s}}\left(\frac{t}{\mu^{2}}, \alpha_{s}\right)\right)^{2}
$$

Each factor $\mathcal{Z}_{\mathbf{1 , R}}^{\mathrm{i}}$ can be thought of as a 'jet' operator, and one may expect these jet operators to combine naturally to yield the divergent parts of the impact factors. We will see below that this is indeed the case.

\section{High-energy color structure and the signature of the amplitude}

One of the key features of high-energy factorization, as exemplified in eq. (1.2), is the 'signature' of reggeized gluon exchange, derived from the expected symmetry of ladder diagrams contributing to high-energy logarithms. We now show that this feature of high-energy amplitudes emerges naturally from infrared factorization, with a simple rearrangement of eq. (2.5). To this end, we must first generalize eq. (1.2) to include the scattering of quarks as well as gluons. In order to do so, we need to take into account the fact that the color factor for quark-quark scattering does not have a definite symmetry property under $s \leftrightarrow u$. In that case, therefore, the symmetric and the antisymmetric parts of the kinematic factor must be weighted differently. Furthermore, we must write the result in a notation compatible with our discussion of infrared factorization, noting that high-energy factorization, 
as described in eq. (1.2), applies only to the exchange of the octet representation in the $t$ channel. Choosing therefore a $t$-channel exchange basis, we can generalize eq. (1.2) as

$$
\begin{gathered}
\mathcal{M}_{\mathrm{rs}}^{[8]}\left(\frac{s}{\mu^{2}}, \frac{t}{\mu^{2}}, \alpha_{s}\right)=2 \pi \alpha_{s} H_{\mathrm{rs}}^{(0),[8]} \\
\times\left\{C_{\mathrm{r}}\left(\frac{t}{\mu^{2}}, \alpha_{s}\right)\left[A_{+}\left(\frac{s}{t}, \alpha_{s}\right)+\kappa_{\mathrm{rs}} A_{-}\left(\frac{s}{t}, \alpha_{s}\right)\right] C_{\mathrm{s}}\left(\frac{t}{\mu^{2}}, \alpha_{s}\right)\right. \\
\left.+\mathcal{R}_{\mathrm{rs}}^{[8]}\left(\frac{s}{\mu^{2}}, \frac{t}{\mu^{2}}, \alpha_{s}\right)+\mathcal{O}\left(\frac{t}{s}\right)\right\},
\end{gathered}
$$

where, as before, the indices r, s label the parton species (quark or gluon), and the Regge trajectory appears in the combinations

$$
A_{ \pm}\left(\frac{s}{t}, \alpha_{s}\right)=\left(\frac{-s}{-t}\right)^{\alpha(t)} \pm\left(\frac{s}{-t}\right)^{\alpha(t)}
$$

with $\kappa_{g g}=\kappa_{q g}=0$, while $\kappa_{q q}=\left(4-N_{c}^{2}\right) / N_{c}^{2}$. In eq. (3.1) we have also introduced a non-factorizing remainder function $\mathcal{R}_{\mathrm{rs}}$, which is expected to receive contributions starting at NNLL and which will be discussed in detail in what follows. Finally, $H_{\mathrm{rs}}^{(0),[8]}$ represents the tree-level amplitude, which depends on the process, and includes the factor $s / t$ which appeared explicitly in eq. (1.2).

In order to accurately match eq. (2.5) with eq. (3.1), the presence of the 'Coulomb phase' factor proportional to $\mathcal{C}_{\text {tot }}$ in eq. (2.5) is crucial. Indeed, using the relation

$$
\mathbf{T}_{s}^{2}+\mathbf{T}_{t}^{2}+\mathbf{T}_{u}^{2}=\mathcal{C}_{\text {tot }}
$$

it is possible to combine the Coulomb phase in eq. (2.5) with the matrix $\widetilde{\mathcal{Z}}$ to define a new infrared matrix

$$
\begin{aligned}
& \widetilde{\mathcal{Z}}_{\mathrm{S}}\left(\frac{s}{t}, \alpha_{s}\right) \equiv \exp \left(-\mathrm{i} \frac{\pi}{2} K\left(\alpha_{s}\right) \mathcal{C}_{\text {tot }}\right) \widetilde{\mathcal{Z}}\left(\frac{s}{t}, \alpha_{s}\right) \\
& \quad=\exp \left\{K\left(\alpha_{s}\right)\left[\left(\log \left(\frac{s}{-t}\right)-\mathrm{i} \frac{\pi}{2}\left(1+\kappa_{\mathrm{rs}}\right)\right) \mathbf{T}_{t}^{2}+\mathrm{i} \frac{\pi}{2}\left(\mathbf{T}_{s}^{2}-\mathbf{T}_{u}^{2}+\kappa_{\mathrm{rs}} \mathbf{T}_{t}^{2}\right)\right]\right\} .
\end{aligned}
$$

Factorizing the matrix $\mathcal{Z}$ in terms of $\widetilde{\mathcal{Z}}_{\mathrm{S}}$ and $\mathcal{Z}_{1, \mathbf{R}}$ provides the most natural description of the dipole formula in the high-energy limit, because it makes more manifest the $s \leftrightarrow u \sim-s$ symmetry property that can be exploited in this regime. Indeed, we can rewrite $\widetilde{\mathcal{Z}}_{\mathrm{S}}$ as

$$
\begin{aligned}
& \widetilde{\mathcal{Z}}_{\mathrm{S}}\left(\frac{s}{t}, \alpha_{s}\right)=\exp \left\{K ( \alpha _ { s } ) \left[\frac { 1 } { 2 } \left[\log \left(\frac{s+\mathrm{i} \eta}{-t}\right)+\log \left(\frac{-s-\mathrm{i} \eta}{-t}\right)\right.\right.\right. \\
& \left.\left.\left.\quad+\kappa_{\mathrm{rs}}\left(\log \left(\frac{-s-\mathrm{i} \eta}{-t}\right)-\log \left(\frac{s+\mathrm{i} \eta}{-t}\right)\right)\right] \mathbf{T}_{t}^{2}+\mathrm{i} \frac{\pi}{2}\left(\mathbf{T}_{s}^{2}-\mathbf{T}_{u}^{2}+\kappa_{\mathrm{rs}} \mathbf{T}_{t}^{2}\right)\right]\right\} .
\end{aligned}
$$

Comparing with the structure of the scattering amplitude in the high-energy limit, as given in eq. (3.1), it is easy to see that at the leading logarithmic level (and at NLL for the real 
part of the amplitude) the terms proportional to $\mathbf{T}_{t}^{2}$ in the exponent of the infrared operator $\widetilde{\mathcal{Z}}_{\mathrm{S}}$ reproduce not only the energy logarithms, $\ln (s /(-t))$, but also the correct symmetry properties under $s \leftrightarrow u$ exchange, that is to say the correct signature of the amplitude (hence the label $S$ attributed to the new infrared matrix $\widetilde{\mathcal{Z}}_{\mathrm{S}}$ ). The breaking of high-energy factorization arises in the first instance from the last term in eq. (3.5), which is a color- and process-dependent phase independent of kinematic invariants. This is in accordance with the expectation that the failure of high-energy factorization should come from the mixing of different color amplitudes: indeed, while the color operator $\mathbf{T}_{t}^{2}$ is diagonal in a $t$-channel color basis, the operator $\mathbf{T}_{s}^{2}-\mathbf{T}_{u}^{2}+\kappa_{\mathrm{rs}} \mathbf{T}_{t}^{2}$ is not, and will induce mixing between different color components of the amplitude. Moreover, one can see that corrections to high-energy factorization must be expected even from the $t$-channel term alone, because of the different way the $s \leftrightarrow u$ symmetry is realised in the Regge factorization formula and in the dipole formula. Indeed, the former consists of a sum of symmetric and anti-symmetric terms, see eq. (3.1), while, focusing on the color diagonal part of the latter, eq. (3.5), proportional to $\mathbf{T}_{t}^{2}$, one can see that the separation between symmetric and anti-symmetric contribution appears in the exponent, so that mixing will be generated upon expansion.

A more detailed analysis of eq. (3.5) readily leads to the expected conclusion that highenergy factorization begins to break down at NNLL level. In order to expand eq. (3.4) in order of increasing logarithmic accuracy, we need to make use of the Zassenhaus formula

$$
\mathrm{e}^{k(X+Y)}=\mathrm{e}^{k X} \mathrm{e}^{k Y} \mathrm{e}^{-\frac{k^{2}}{2}[X, Y]} \mathrm{e}^{\frac{k^{3}}{3 !}(2[Y,[X, Y]]+[X,[X, Y]])} \mathrm{e}^{\mathcal{O}\left(k^{4}\right)},
$$

with the identifications

$$
\begin{aligned}
k & =K\left(\alpha_{s}\right), \\
X & =\left(\log \left(\frac{s}{-t}\right)-\mathrm{i} \frac{\pi}{2}\left(1+\kappa_{\mathrm{rs}}\right)\right) \mathbf{T}_{t}^{2}, \\
Y & =\mathrm{i} \frac{\pi}{2}\left(\mathbf{T}_{s}^{2}-\mathbf{T}_{u}^{2}+\kappa_{\mathrm{rs}} \mathbf{T}_{t}^{2}\right) .
\end{aligned}
$$

Clearly, since the function $K\left(\alpha_{s}\right)$ begins at $\mathcal{O}\left(\alpha_{s}\right)$, all leading logarithms are generated by the first exponential in eq. (3.6), $\mathrm{e}^{k X}$. Next-to-leading logarithms arise from the infinite sequence of multiple commutators involving only one power of $Y$, and an arbitrary number of powers of $X$, and so forth. In order to continue the analysis, it is convenient to introduce a shorthand notation for color operators. We define

$$
\begin{aligned}
\mathbf{O}_{t} & =\mathbf{T}_{t}^{2} \\
\mathbf{O}_{s-u} & =\mathbf{T}_{s}^{2}-\mathbf{T}_{u}^{2}+\kappa_{\mathrm{rs}} \mathbf{T}_{t}^{2}=2 \mathbf{T}_{s}^{2}+\left(1+\kappa_{\mathrm{rs}}\right) \mathbf{T}_{t}^{2}-\mathcal{C}_{\mathrm{tot}} \\
\mathbf{O}_{t, s} & =\left[\mathbf{T}_{t}^{2}, \mathbf{T}_{s}^{2}\right] \\
\mathbf{O}_{t, t, s} & =\left[\mathbf{T}_{t}^{2},\left[\mathbf{T}_{t}^{2}, \mathbf{T}_{s}^{2}\right]\right] \\
\mathbf{O}_{s, t, s} & =\left[\mathbf{T}_{s}^{2},\left[\mathbf{T}_{t}^{2}, \mathbf{T}_{s}^{2}\right]\right]
\end{aligned}
$$

with the natural generalizations to higher order commutators. In terms of these color operators, the exponents of the various factors on the right-hand side of eq. (3.6) read

$$
k X=K\left(\alpha_{s}\right)\left[\log \left(\frac{s}{-t}\right)-\mathrm{i} \frac{\pi}{2}\left(1+\kappa_{\mathrm{rs}}\right)\right] \mathbf{O}_{t},
$$




$$
\begin{aligned}
k Y= & \mathrm{i} \frac{\pi}{2} K\left(\alpha_{s}\right) \mathbf{O}_{s-u} \\
-\frac{k^{2}}{2}[X, Y]= & K^{2}\left(\alpha_{s}\right)\left[-\mathrm{i} \frac{\pi}{2} \log \left(\frac{s}{-t}\right)-\frac{\pi^{2}}{4}\left(1+\kappa_{\mathrm{rs}}\right)\right] \mathbf{O}_{t, s} \\
\frac{k^{3}}{3 !}(2[Y,[X, Y]]+[X,[X, Y]])= & \frac{K^{3}\left(\alpha_{s}\right)}{3 !}\left\{\left[-2 \pi^{2} \log \left(\frac{s}{-t}\right)+\mathrm{i} \pi^{3}\left(1+\kappa_{\mathrm{rs}}\right)\right] \mathbf{O}_{s, t, s}\right. \\
& \left.+\left[\mathrm{i} \pi \log ^{2}\left(\frac{s}{-t}\right)+\mathrm{i} \frac{\pi^{3}}{4}\left(1+\kappa_{\mathrm{rs}}\right)^{2}\right] \mathbf{O}_{t, t, s}\right\} .
\end{aligned}
$$

Starting with eq. (3.10), one can verify that all color operators appearing at NLL, namely $\mathbf{O}_{s-u}, \mathbf{O}_{t, s}, \mathbf{O}_{t, t, s}$, and more generally $\mathbf{O}_{t, \ldots, t, s}$ give vectors with a vanishing octet component, when acting on pure octet matrix elements, and thus in particular on the tree-level quark and gluon amplitudes at leading power in $|t| / s$. Indeed, one finds that

$$
\begin{aligned}
{\left[\mathbf{O}_{s-u}\right]^{[8],[8]} } & =\left[2 \mathbf{T}_{s}^{2}+\left(1+\kappa_{\mathrm{rs}}\right) \mathbf{T}_{t}^{2}-\mathcal{C}_{\mathrm{tot}}\right]^{[8],[8]} \\
& =2\left[\mathbf{T}_{s}^{2}\right]^{[8],[8]}+\left(1+\kappa_{\mathrm{rs}}\right) C_{A}-\mathcal{C}_{\mathrm{tot}}=0, \\
{\left[\mathbf{O}_{t, \ldots, t, s}\right]^{[8],[8]} } & =\left[\mathbf{T}_{t}^{2},\left[\mathbf{T}_{t}^{2}, \ldots,\left[\mathbf{T}_{t}^{2}, \mathbf{T}_{s}^{2}\right] \ldots\right]\right]^{[8],[8]}=0 .
\end{aligned}
$$

It is important to notice that, while the second identity in eq. (3.11) is a simple consequence of the fact that $\mathbf{T}_{t}^{2}$ can be replaced by its eigenvalues in a $t$-channel basis, the first identity is non-trivial and to some extent surprising: it is a necessary condition for the reggeization of next-to-leading logarithms, and, as such, it is a consequence of known properties of the high-energy limit; it embodies symmetry properties of the amplitudes, related to crossing symmetry, and indeed it could be used as a definition of the symmetry factor $\kappa_{\mathrm{rs}}$; finally, we have explicitly checked that it is verified for quark and gluon amplitudes with the choice of color basis described in appendix A. On the other hand, operators like $\mathbf{O}_{s, t, s}$ in eq. (3.10), which induce non-trivial mixing between different color amplitudes, appear only starting at NNLL, so that only at this level one expects a breakdown of high-energy factorization.

\section{Comparing infrared and high-energy factorizations for octet exchange}

We now get to the central goal of this paper, which is the comparison of the two different factorizations that we have described, given respectively by eq. (2.2), together with the information on the high-energy limit collected in section 2, and eq. (3.1). The two factorizations differ substantially in scope and accuracy: infrared factorization for amplitudes organizes only infrared divergent contributions, but it is exact to all orders in perturbation theory; furthermore, the high-energy limit of the infrared operator $\mathcal{Z}$ discussed in section 3 is accurate to leading power in $t / s$. On the other hand, high-energy factorization targets finite contributions to the amplitude, but it is only expected to work to a limited logarithmic accuracy. Comparing the two approaches, we are going to extract constraints on the amplitude, which will eventually enable us to make predictions based on one of the two factorizations, when the second one is not applicable. 
Our first task is to systematically expand our factorized expressions in powers of the coupling and, where present, of the high-energy logarithm, $\ln (s /|t|)$. For example, to leading power in $t / s$, each color component of the amplitude can be organized as a double expansion, writing

$$
\mathcal{M}^{[j]}\left(\frac{s}{\mu^{2}}, \frac{t}{\mu^{2}}, \alpha_{s}\right)=4 \pi \alpha_{s} \sum_{n=0}^{\infty} \sum_{i=0}^{n}\left(\frac{\alpha_{s}}{\pi}\right)^{n} \ln ^{i}\left(\frac{s}{-t}\right) M^{(n), i,[j]}\left(\frac{t}{\mu^{2}}\right),
$$

with corrections suppressed by powers of $t / s$. The color components of the finite hard vector $\mathcal{H}$ can be similarly expanded as

$$
\mathcal{H}^{[j]}\left(\frac{s}{\mu^{2}}, \frac{t}{\mu^{2}}, \alpha_{s}\right)=4 \pi \alpha_{s} \sum_{n=0}^{\infty} \sum_{i=0}^{n}\left(\frac{\alpha_{s}}{\pi}\right)^{n} \ln ^{i}\left(\frac{s}{-t}\right) H^{(n), i,[j]}\left(\frac{t}{\mu^{2}}\right) .
$$

In this notation, since the tree-level matrix element has no logarithms, as well as obviously no divergences, one has $H^{(0),[8]}=H^{(0), 0,[8]}$. The octet remainder $\mathcal{R}_{\mathrm{rs}}^{[8]}$ defined in eq. (3.1) is also expanded as

$$
\mathcal{R}_{\mathrm{rS}}^{[8]}\left(\frac{s}{\mu^{2}}, \frac{t}{\mu^{2}}, \alpha_{s}\right)=\sum_{n=2}^{\infty} \sum_{k=0}^{n-2}\left(\frac{\alpha_{s}}{\pi}\right)^{n} \ln ^{k}\left(\frac{s}{-t}\right) R_{\mathrm{rs}}^{(n), k,[8]}\left(\frac{t}{\mu^{2}}\right) .
$$

Notice that we have included the information that the remainder function must start at NNLL and at the two-loop level. In principle, there is a finite, logarithmically subleading ambiguity in the definition of the remainder function $\mathcal{R}_{\mathrm{rs}}^{[8]}$ : we will see, however, that the knowledge of the structure of the amplitude which comes from infrared factorization suggests a natural choice of 'high-energy factorization scheme', and therefore a natural choice for the non-factorizing remainder.

Quantities that do not depend on the center-of-mass energy $s$ are just expanded in perturbation theory. For example for the Regge trajectory and for the impact factors we write

$$
\alpha(t)=\sum_{n=1}^{\infty}\left(\frac{\alpha_{s}}{\pi}\right)^{n} \alpha^{(n)}(t), \quad C_{\mathrm{r}}\left(\frac{t}{\mu^{2}}, \alpha_{s}\right)=\sum_{n=0}^{\infty}\left(\frac{\alpha_{s}}{\pi}\right)^{n} C_{\mathrm{r}}^{(n)}\left(\frac{t}{\mu^{2}}\right),
$$

and we choose the normalization so that $C_{\mathrm{r}}^{(0)}=1$. In practice, in all subsequent calculations we will set the renormalization scale $\mu^{2}=-t$, so that the perturbative coefficients of these functions will be just numbers. Notice also that in the literature on high-energy amplitudes $[4,16,45]$ certain universal $\epsilon$-dependent factors are reabsorbed in the coupling, and the perturbative expansion is effectively in powers of a shifted coupling $\tilde{\alpha}_{s}=c_{\Gamma} \alpha_{s}$, where

$$
c_{\Gamma}=\mathrm{e}^{\varepsilon \gamma_{\mathrm{E}}} \frac{\Gamma(1-\varepsilon)^{2} \Gamma(1+\varepsilon)}{\Gamma(1-2 \varepsilon)} .
$$

We will not follow this practice in our calculations below, since we want our results to be expressed in terms of the standard $\overline{M S}$ coupling, to be readily comparable with finiteorder calculations. Since the two definitions begin to differ at $\mathcal{O}\left(\varepsilon^{2}\right)$, some of our results for subleading poles at high-orders would change if the other scheme was adopted. That 
being said, we find that, in several instances, reabsorbing the one-loop factor $c_{\Gamma}$ proves remarkably efficient in simplifying the resulting expressions, even beyond NLL accuracy, where one might expect such a substitution to be helpful. Whenever such a simplification occurs, we will note it explicitly.

We now proceed with our comparison, order by order, beginning at one loop, where everything is well known, in order to set up our convention and illustrate our procedure in a simple setting.

\subsection{One-loop matrix elements}

We begin by expanding eqs. (2.2) and (3.1), to first order in $\alpha_{s}$. For simplicity, we will omit the parton indices $\mathrm{r}, \mathrm{s}$ whenever they are not specifically needed. Infrared factorization yields the expressions

$$
\begin{aligned}
& M^{(1), 0}=\left\{Z_{1, \mathbf{R}}^{(1)}+\mathrm{i} \frac{\pi}{2} K^{(1)}\left[\mathbf{O}_{s-u}-\left(1+\kappa_{\mathrm{rs}}\right) \mathbf{O}_{t}\right]\right\} H^{(0)}+H^{(1), 0}, \\
& M^{(1), 1}=K^{(1)} \mathbf{O}_{t} H^{(0)}+H^{(1), 1}
\end{aligned}
$$

which are still vectors in color space. For the octet component of these vectors, high-energy factorization provides the expressions

$$
\begin{aligned}
& M^{(1), 0,[8]}=\left[C_{a}^{(1)}+C_{b}^{(1)}-\mathrm{i} \frac{\pi}{2}\left(1+\kappa_{\mathrm{rs}}\right) \alpha^{(1)}\right] H^{(0),[8]}, \\
& M^{(1), 1,[8]}=\alpha^{(1)} H^{(0),[8]} .
\end{aligned}
$$

One of the constraints of Regge factorization is the fact that the Regge trajectory and the impact factors are required to be real: in other words, the imaginary part of the amplitude is completely determined by the signature properties under the exchange $s \leftrightarrow u$, as given in eq. (3.1) and in eq. (3.2). There is therefore interesting information to be extracted about the imaginary parts of the amplitude when comparing results such as eq. (4.6) and eq. (4.7). Comparing first one-loop terms proportional to $\ln (s /(-t))$, and noting that the second of eqs. (4.7) is real, we immediately see that

$$
\operatorname{Im}\left[H_{\mathrm{rs}}^{(1), 1,[8]}\right]=0 .
$$

In addition, it is known [45] that

$$
\operatorname{Re}\left[H_{\mathrm{rs}}^{(1), 1,[8]}\right]=\mathcal{O}(\varepsilon) .
$$

This simple one-loop result, as we will see, bootstraps to higher orders and has important consequences on finite parts of higher-order amplitudes. To begin with, we can now write the one-loop Regge trajectory as

$$
\alpha^{(1)}=\frac{K^{(1)} \mathbf{T}_{t}^{2} H^{(0)}}{H^{(0),[8]}}+\mathcal{O}(\varepsilon) .
$$

In the high-energy limit, for all parton species, the tree-level amplitude at leading power in $|t| / s$ is a pure color octet in the $t$-channel, and therefore it is an eigenvector of the $\mathbf{T}_{t}^{2}$ operator with eigenvalue $C_{A}$. As expected, the Regge trajectory then becomes

$$
\alpha^{(1)}=C_{A} K^{(1)}+\mathcal{O}(\varepsilon)=\frac{C_{A}}{2 \varepsilon}+\mathcal{O}(\varepsilon),
$$


which confirms the universality of the one-loop Regge trajectory [46-52] to $\mathcal{O}(\varepsilon)$. Notice that in the context of one-loop calculations these $\mathcal{O}(\varepsilon)$ terms can be safely neglected. Here however we allow for the possibility that $\mathcal{O}(\varepsilon)$ contributions might interfere with higherorder poles coming from the expansion of the infrared operators beyond one loop. In the present case, $\mathcal{O}(\varepsilon)$ contributions come exclusively from the factor $c_{\Gamma}$ in eq. (4.5).

Turning to non-logarithmic contributions to the matrix elements in eqs. (4.6) and (4.7), and comparing their imaginary parts, we obtain

$$
\begin{aligned}
\operatorname{Im}\left[H_{\mathrm{rs}}^{(1), 0,[8]}\right]= & -\frac{\pi}{2}\left(1+\kappa_{\mathrm{rs}}\right) \alpha^{(1)} H_{\mathrm{rs}}^{(0),[8]} \\
& -\frac{\pi}{2} K^{(1)}\left\{\left[\mathbf{O}_{s-u}-\left(1+\kappa_{\mathrm{rs}}\right) \mathbf{O}_{t}\right] H^{(0)}\right\}^{[8]} .
\end{aligned}
$$

Using the form of the Regge trajectory, eq. (4.10), and the identity in eq. (3.11), it is easy to see that

$$
\operatorname{Im}\left[H_{\mathrm{rs}}^{(1), 0,[8]}\right]=-\frac{\pi}{2}\left(1+\kappa_{\mathrm{rs}}\right) \operatorname{Re}\left[H_{\mathrm{rs}}^{(1), 1,[8]}\right]=\mathcal{O}(\varepsilon)
$$

Notice that the vanishing of the octet-octet component of the operator $\mathbf{O}_{s-u}$, noted in eq. (3.11), is crucial for the compatibility of eq. (4.12) with infrared factorization: if that matrix element were to be non-vanishing, the right-hand side of eq. (4.12) would have a leftover uncancelled IR divergence, which would be incompatible with the definition of $\mathcal{H}$ as the finite remainder of the matrix element. A combined consequence of Reggeization and infrared factorization is thus that imaginary parts of one-loop amplitudes are completely fixed in terms of the real parts. The key element which guarantees that this can happen is precisely the fact that the operator $\mathbf{O}_{s-u}$, appearing in eq. (4.12), gives a vector with a vanishing octet component, when applied to an octet amplitude, as shown in eq. (3.11).

Finally, comparing the real parts of the non-logarithmic contributions to eqs. (4.6) and (4.7), and considering separately the quark-quark and the gluon-gluon scattering amplitudes, we can determine the respective impact factors. One finds that

$$
C_{\mathrm{r}}^{(1)}=\frac{1}{2} Z_{1, \mathbf{R}, \mathrm{rr}}^{(1)}+\frac{1}{2} \widehat{H}_{\mathrm{rr}}^{(1), 0,[8]},
$$

where we defined $\widehat{H}_{\mathrm{rs}}^{(m), n,[J]}=H_{\mathrm{rs}}^{(m), n,[J]} / H_{\mathrm{rs}}^{(0),[8]}$. The coefficients $Z_{1, \mathbf{R}, \mathrm{r}}^{(1)}$ can be expressed in terms of known anomalous dimensions, given in eq. (B.3) and eq. (B.4), while hard parts can be read off eqs. (C.1), (C.4), (C.7), and (C.9). This gives the one loop impact factors

$$
\begin{aligned}
C_{q}^{(1)} & =-\frac{1}{2} C_{F}\left(\frac{1}{\varepsilon^{2}}+\frac{3}{2 \varepsilon}\right)+N_{c}\left(\frac{13}{72}+\frac{7}{8} \zeta(2)\right)+\frac{1}{N_{c}}\left(1-\frac{1}{8} \zeta(2)\right)-\frac{5}{36} n_{f}+\mathcal{O}(\varepsilon), \\
C_{g}^{(1)} & =-\frac{1}{2 \varepsilon^{2}} N_{c}-\frac{b_{0}}{4 \varepsilon}-N_{c}\left(\frac{67}{72}-\zeta(2)\right)+\frac{5}{36} n_{f}+\mathcal{O}(\varepsilon) .
\end{aligned}
$$

Having determined both impact factors, one can finally verify the consistency of Regge factorization, by constructing the high-energy quark-gluon scattering amplitude. One can use the fact that, by virtue of eq. (2.11), the color-singlet infrared operators $Z_{1, \mathbf{R}, \text { rs }}$ satisfy

$$
Z_{1, \mathbf{R}, q g}^{(1)}=\frac{1}{2}\left[Z_{1, \mathbf{R}, q q}^{(1)}+Z_{1, \mathbf{R}, g g}^{(1)}\right]
$$


With this result, borrowed from infrared factorization, requiring Regge factorization leaves a constraint on the hard parts of the amplitudes, which must satisfy

$$
\operatorname{Re}\left(\widehat{H}_{q g}^{(1), 0,[8]}\right)=\frac{1}{2}\left[\operatorname{Re}\left(\widehat{H}_{g g}^{(1), 0,[8]}\right)+\operatorname{Re}\left(\widehat{H}_{q q}^{(1), 0,[8]}\right)\right] .
$$

Our explicit results for hard parts, extracted from ref. [53], are given in appendix C, and they are easily verified to satisfy eq. (4.17).

\section{$4.2 \quad$ Two-loop matrix elements}

Repeating the procedure at two loops, one finds more interesting results and, as we describe below, at the level of non-logarithmic terms one begins to see the breakdown of the highenergy factorization, as given in eq. (3.1).

As above, we begin by expanding eq. (2.2), this time to second order in $\alpha_{s}$. We find

$$
\begin{aligned}
M^{(2), 0}= & {\left[\mathrm{i} \frac{\pi}{2}\left(K^{(2)}+K^{(1)} Z_{1, \mathbf{R}}^{(1)}\right)\left(\mathbf{O}_{s-u}-\left(1+\kappa_{\mathrm{rs}}\right) \mathbf{O}_{t}\right)+Z_{1, \mathbf{R}}^{(2)}\right.} \\
\left.-\frac{\pi^{2}}{8}\left(K^{(1)}\right)^{2}\left(\mathbf{O}_{s-u}^{2}+2 \mathbf{O}_{t, s}\left(1+\kappa_{\mathrm{rs}}\right)-2 \mathbf{O}_{t} \mathbf{O}_{s-u}\left(1+\kappa_{\mathrm{rs}}\right)+\mathbf{O}_{t}^{2}\left(1+\kappa_{\mathrm{rs}}\right)^{2}\right)\right] H^{(0)} & +\left[\mathrm{i} \frac{\pi}{2} K^{(1)}\left(\mathbf{O}_{s-u}-\left(1+\kappa_{\mathrm{rs}}\right) \mathbf{O}_{t}\right)+Z_{1, \mathbf{R}}^{(1)}\right] H^{(1), 0}+H^{(2), 0}, \\
M^{(2), 1}= & {\left[\mathrm{i} \frac{\pi}{2}\left(K^{(1)}\right)^{2}\left(-\mathbf{O}_{t, s}+\mathbf{O}_{t} \mathbf{O}_{s-u}-\left(1+\kappa_{\mathrm{rs}}\right) \mathbf{O}_{t}^{2}\right)+K^{(1)} Z_{1, \mathbf{R}}^{(1)} \mathbf{O}_{t}+K^{(2)} \mathbf{O}_{t}^{2}\right] H^{(0)} } \\
& +K^{(1)} \mathbf{O}_{t} H^{(1), 0}+\left[\mathrm{i} \frac{\pi}{2} K^{(1)}\left(\mathbf{O}_{s-u}-\left(1+\kappa_{\mathrm{rs}}\right) \mathbf{O}_{t}\right)+Z_{1, \mathbf{R}}^{(1)}\right] H^{(1), 1}+H^{(2), 1}, \\
M^{(2), 2}= & \frac{\left(K^{(1)}\right)^{2}}{2} \mathbf{O}_{t}^{2} H^{(0)}+K^{(1)} \mathbf{O}_{t} H^{(1), 1}+H^{(2), 2},
\end{aligned}
$$

where each expression is a vector in color space. For the octet component, we can also expand eq. (3.1) to second order in $\alpha_{s}$, yielding

$$
\begin{aligned}
M_{\mathrm{rs}}^{(2), 0,[8]}= & \left\{C_{\mathrm{r}}^{(1)} C_{\mathrm{s}}^{(1)}+C_{\mathrm{r}}^{(2)}+C_{\mathrm{s}}^{(2)}-\mathrm{i} \frac{\pi}{2}\left(1+\kappa_{\mathrm{rs}}\right)\left[\left(C_{\mathrm{r}}^{(1)}+C_{\mathrm{s}}^{(1)}\right) \alpha^{(1)}+\alpha^{(2)}\right]\right. \\
& \left.-\frac{\pi^{2}}{4}\left(1+\kappa_{\mathrm{rs}}\right)\left(\alpha^{(1)}\right)^{2}+\frac{1}{2} R_{\mathrm{rs}}^{(2), 0,[8]}\right\} H_{\mathrm{rs}}^{(0),[8]}, \\
M_{\mathrm{rs}}^{(2), 1,[8]}= & {\left[\left(C_{\mathrm{r}}^{(1)}+C_{\mathrm{s}}^{(1)}\right) \alpha^{(1)}-\mathrm{i} \frac{\pi}{2}\left(1+\kappa_{\mathrm{rs}}\right)\left(\alpha^{(1)}\right)^{2}+\alpha^{(2)}\right] H_{\mathrm{rs}}^{(0),[8]} } \\
M_{\mathrm{rs}}^{(2), 2,[8]}= & \frac{1}{2}\left(\alpha^{(1)}\right)^{2} H_{\mathrm{rs}}^{(0),[8]}
\end{aligned}
$$

where in the first equation, which contains the non-logarithmic NNLL contribution at two loops, we allow for a non-factorizing remainder, as in eq. (4.3).

Comparing the coefficients of the leading logarithms, that is the expressions at the bottom of eqs. (4.18) and (4.19), one readily verifies that the coefficient of the highest power of the energy logarithm is determined by the one-loop result, and in particular that 
the imaginary part of the hard matrix element vanishes, while the real part is of higher order in $\varepsilon$,

$$
\begin{aligned}
\operatorname{Im}\left[H_{\mathrm{rs}}^{(2), 2,[8]}\right] & =0 \\
\operatorname{Re}\left[H_{\mathrm{rs}}^{(2), 2,[8]}\right] & =\frac{\operatorname{Re}\left[H_{\mathrm{rs}}^{(1), 1,[8]}\right]^{2}}{2 H_{\mathrm{rs}}^{(0),[8]}}=\mathcal{O}\left(\varepsilon^{2}\right),
\end{aligned}
$$

as expected from high-energy factorization.

At the level of single logarithms at two loops, that is the middle expressions in eqs. (4.18) and (4.19), we expect to recover the two-loop Regge trajectory, and we expect high-energy factorization to continue holding. Indeed, one finds that the imaginary part yields,

$$
\begin{aligned}
\operatorname{Im}\left[H_{\mathrm{rs}}^{(2), 1,[8]]}=\right. & -\frac{\pi}{2}\left(K^{(1)}\right)^{2}\left[\left(-\mathbf{O}_{t, s}+\mathbf{O}_{t} \mathbf{O}_{s-u}-\left(1+\kappa_{\mathrm{rs}}\right) \mathbf{O}_{t}^{2}\right) H_{\mathrm{rs}}^{(0)}\right]^{[8]} \\
& -\frac{\pi}{2} K^{(1)}\left[\left(\mathbf{O}_{s-u}-\left(1+\kappa_{\mathrm{rs}}\right) \mathbf{O}_{t}\right) \operatorname{Re}\left[H_{\mathrm{rs}}^{(1), 1}\right]\right]^{[8]}-Z_{1, \mathbf{R}}^{(1)} \operatorname{Im}\left[H_{\mathrm{rs}}^{(1), 1,[8]}\right] \\
& -\frac{\pi}{2}\left(1+\kappa_{\mathrm{rs}}\right)\left(\alpha^{(1)}\right)^{2} H_{\mathrm{rs}}^{(0),[8]} .
\end{aligned}
$$

Substituting the one-loop Regge trajectory, eq. (4.10), it is easy to see that eq. (4.21) reduces to

$$
\operatorname{Im}\left[H_{\mathrm{rs}}^{(2), 1,[8]}\right]=-\frac{\pi}{2}\left(1+\kappa_{\mathrm{rs}}\right)\left(\widehat{H}_{\mathrm{rs}}^{(1), 1,[8]}\right)^{2} H_{\mathrm{rs}}^{(0),[8]}=\mathcal{O}\left(\varepsilon^{2}\right),
$$

which is easy to understand, using again eq. (3.11), and remembering that $\operatorname{Im}\left[H_{\mathrm{rs}}^{(1), 1}\right]=0$. Once again, eq. (3.11) is crucial for consistency with infrared factorization.

The two loop Regge trajectory $[4,54-57]$ is determined from the real part of the single logarithms. By replacing $\operatorname{Im}\left[H^{(1), 1}\right]=0$ in the expansion of the amplitude and introducing the explicit results for the one loop coefficients in the Regge formula we have

$$
\alpha^{(2)}=C_{A} K^{(2)}+\operatorname{Re}\left[\widehat{H}_{\mathrm{rs}}^{(2), 1,[8]}\right]+\mathcal{O}(\varepsilon) .
$$

As expected, the divergent part of the two loop Regge traiectory is entirely given by the integral of the two loop cusp anomalous dimension and is independent of the specific scattering process considered. This is again in perfect agreement with high-energy factorization. The requirement that the finite part of the two-loop Regge trajectory found in the $q q$ and $g g$ process be independent of the scattering process implies an identity for the real part of the amplitude. The requirement is that

$$
\operatorname{Re}\left[\widehat{H}_{g g}^{(2), 1,[8]}\right]=\operatorname{Re}\left[\widehat{H}_{q g}^{(2), 1,[8]}\right]=\operatorname{Re}\left[\widehat{H}_{q q}^{(2), 1,[8]}\right],
$$

which is indeed satisfied. We directly check this condition by normalizing eqs. (C.5), (C.11) and (C.17) with the corresponding tree level amplitudes, given respectively in (C.1), (C.7) and (C.13). This gives the universal result

$$
\operatorname{Re}\left[\widehat{H}_{\mathrm{rs}}^{(2), 1,[8]}\right]=\left(\frac{101}{108}-\frac{\zeta(3)}{8}\right) N_{c}^{2}-\frac{7}{54} N_{c} n_{f}
$$

which, when inserted in eq. (4.23), reproduces the well-known result of refs. [4, 54-57]. 
Turning finally to non-logarithmic terms, given in the top expressions of eqs. (4.18) and (4.19), we see that their imaginary part yields

$$
\begin{aligned}
\operatorname{Im}\left[H_{\mathrm{rs}}^{(2), 0,[8]}\right]= & -\frac{\pi}{2}\left(K^{(2)}+K^{(1)} Z_{1, \mathbf{R}}^{(1)}\right)\left[\left(\mathbf{O}_{s-u}-\left(1+\kappa_{\mathrm{rs}}\right) \mathbf{O}_{t}\right) H^{(0)}\right]^{[8]} \\
& -\frac{\pi}{2} K^{(1)}\left[\left(\mathbf{O}_{s-u}-\left(1+\kappa_{\mathrm{rs}}\right) \mathbf{O}_{t}\right) \operatorname{Re}\left[H^{(1), 0}\right]\right]^{[8]}+Z_{1, \mathbf{R}}^{(1)} \operatorname{Im}\left[H^{(1), 0,[8]}\right] \\
& -\frac{\pi}{2}\left(1+\kappa_{\mathrm{rs}}\right)\left[\left(C_{\mathrm{r}}^{(1)}+C_{\mathrm{s}}^{(1)}\right) \alpha^{(1)}+\alpha^{(2)}\right] H_{\mathrm{rs}}^{(0),[8]}
\end{aligned}
$$

A remarkable consequence of eq. (4.26) is that high-energy factorization still works at NNLL for the imaginary part of the octet amplitude. This is a consequence of the fact that the effects of the color mixing operators on the imaginary part of the octet amplitude are delayed by one order as compared to the real part of the amplitude. Specifically, we see that the only color mixing operator appearing in eq. (4.26) is again $\mathbf{O}_{s-u}$, which, as noted above, gives a vanishing octet component when acting on a color octet state. The remaining terms in the first two lines of eq. (4.26) combine to cancel exactly the contribution given in the third line, leaving the finite remainder

$$
\operatorname{Im}\left[H_{\mathrm{rs}}^{(2), 0,[8]}\right]=-\frac{\pi}{2}\left(1+\kappa_{\mathrm{rs}}\right) \operatorname{Re}\left[H_{\mathrm{rs}}^{(2), 1,[8]}\right],
$$

which is in agreement with the corresponding coefficients of the two-loop amplitudes [4], given in eqs. (C.6), (C.12) and (C.18).

When we consider the real part of NNLL contributions at two loops, given by the top expressions of eqs. (4.18) and (4.19), we finally begin to see the non-universal effects that bring about the breaking of high-energy factorization. Basically, the breaking of universality can be traced back to three factors.

- The appearance of the operator $\left(\mathbf{O}_{s-u}\right)^{2}$, which, acting on $H^{(0)}$, gives a non-vanishing octet component, in contrast to $\mathbf{O}_{s-u}, \mathbf{O}_{t, s}$, and in general $\mathbf{O}_{t, \ldots, t, s}$ : these operators, when applied to a pure color octet amplitude, give a vector with a vanishing octet component. To be more explicit, one has

$$
\left[\left(\mathbf{O}_{s-u}\right)^{2} H^{(0)}\right]^{[8]}=\sum_{[i]}\left[\mathbf{O}_{s-u}\right]^{[8],[i]}\left[\mathbf{O}_{s-u}\right]^{[i],[8]} H^{(0),[8]} \neq 0 .
$$

- A mismatch between the Regge factorization formula and the high-energy limit of the infrared factorization formula in the octet channel itself: this can be easily seen by noting that

$$
-\frac{\pi^{2}}{8}\left(K^{(1)}\right)^{2}\left[\mathbf{O}_{t}^{2}\left(1+\kappa_{\mathrm{rs}}\right)^{2} H^{(0)}\right]^{[8]} \neq-\frac{\pi^{2}}{4}\left(1+\kappa_{\mathrm{rs}}\right)\left(\alpha^{(1)}\right)^{2} H_{\mathrm{rs}}^{(0),[8]} .
$$

- The contributions of the other color components of the amplitude. Starting at two loops, one needs to take into account the effect of the operators $\mathbf{O}_{s-u}, \mathbf{O}_{t, s}$, and in general $\mathbf{O}_{t, \ldots, t, s}$ on the non-octet components of the amplitude, which are zero at tree level, but have contributions starting at one loop. For instance, in eq. (4.26) one sees 
that the color octet amplitude receives a contribution proportional to the one-loop imaginary part of the sub-leading color amplitudes, through

$$
\left[\mathbf{O}_{s-u} \operatorname{Im}\left[H_{\mathrm{rs}}^{(1), 0}\right]\right]^{[8]} \neq 0
$$

As we will see below, this last effect is subtle, because it involves terms which are at least $\mathcal{O}(\varepsilon)$ from $\operatorname{Im}\left[H_{\mathrm{rs}}^{(1), 0}\right]$, and it can be made to disappear by expanding the amplitude in powers of $\tilde{\alpha}_{s}=c_{\Gamma} \alpha_{s}$.

In general, $\mathbf{O}_{s-u}, \mathbf{O}_{t, s}$ and the factor $\kappa_{\mathrm{rs}}$ depend on the scattering process through color correlations and through the species of the incoming partons, so we expect that such terms will break the universality of high-energy factorization. Our proposal is to identify all universality-breaking terms and include them in the definition of the remainder function $R^{(2), 0,[8]}$. The analysis of infrared singularities is crucial to pinpoint the non-universal contribution. Indeed, if we replace the one loop impact factor and Regge trajectory eqs. (4.14) and (4.10) in eq. (4.19), and we compare it with the real part of $M_{\mathrm{rr}}^{(2), 0,[8]}$ obtained from eq. (4.18), after using the identities in eq. (3.11) and eq. (4.13), we get an equation for the sum of impact factors and remainder functions, of the form

$$
\begin{aligned}
\left(2 C_{\mathrm{rr}}^{(2)}+\frac{R_{\mathrm{rr}}^{(2), 0,[8]}}{2}\right) H_{\mathrm{rr}}^{(0),[8]=} & Z_{1, \mathbf{R}, \mathrm{rr}}^{(2)}-\frac{1}{4}\left(Z_{1, \mathbf{R}, \mathrm{rr}}^{(1)}\right)^{2}+\frac{1}{2} Z_{1, \mathbf{R}, \mathrm{rr}}^{(1)} \operatorname{Re}\left[\widehat{H}_{\mathrm{rr}}^{(1), 0,[8]}\right] \\
& \left.+\operatorname{Re}\left[\widehat{H}_{\mathrm{rr}}^{(2), 0,[8]}\right]-\frac{1}{4}\left(\operatorname{Re}\left[\widehat{H}_{\mathrm{rr}}^{(1), 0,[8]}\right]\right)^{2}\right] H_{\mathrm{rr}}^{(0),[8]} \\
& -\frac{\pi}{2} K^{(1)}\left\{\frac{\pi K^{(1)}}{4}\left[\left(\mathbf{O}_{s-u}^{2}-\mathbf{O}_{t}^{2}\left(1-\kappa_{\mathrm{rr}}^{2}\right)\right) H_{\mathrm{rr}}^{(0)}\right]^{[8]}\right. \\
& \left.+\left[\mathbf{O}_{s-u} \operatorname{Im}\left[H_{\mathrm{rr}}^{(1), 0}\right]\right]^{[8]}-\frac{\pi N_{c}}{2}\left(1-\kappa_{\mathrm{rr}}^{2}\right) \operatorname{Re}\left[H_{\mathrm{rr}}^{(1), 1,[8]}\right]\right\} .
\end{aligned}
$$

According to the considerations above, we assign all terms containing the operator $\mathbf{O}_{s-u}$ and the factor $\kappa_{\mathrm{rs}}$ to the remainder function, while all the other contributions will define the impact factors. We write then

$$
\begin{aligned}
R_{\mathrm{rs}}^{(2), 0,[8]}= & -\frac{\pi^{2}}{4}\left(K^{(1)}\right)^{2} \frac{1}{H_{\mathrm{rs}}^{(0),[8]}}\left[\left(\mathbf{O}_{s-u}^{2}-\mathbf{O}_{t}^{2}\left(1-\kappa_{\mathrm{rs}}^{2}\right)\right) H_{\mathrm{rs}}^{(0)}\right]^{[8]} \\
& -\frac{\pi K^{(1)}}{H_{\mathrm{rs}}^{(0),[8]}}\left[\mathbf{O}_{s-u} \operatorname{Im}\left[H_{\mathrm{rs}}^{(1), 0}\right]\right]^{[8]}+\frac{\pi^{2}}{2} K^{(1)} N_{c}\left(1-\kappa_{\mathrm{rs}}^{2}\right) \operatorname{Re}\left[\widehat{H}_{\mathrm{rs}}^{(1), 1,[8]}\right],
\end{aligned}
$$

and

$$
\begin{aligned}
C_{\mathrm{r}}^{(2)}= & -\frac{1}{8}\left(Z_{1, \mathbf{R}, \mathrm{rr}}^{(1)}\right)^{2}+\frac{1}{2} Z_{1, \mathbf{R}, \mathrm{rr}}^{(2)}+\frac{1}{4} Z_{1, \mathbf{R}, \mathrm{rr}}^{(1)} \operatorname{Re}\left[\widehat{H}_{\mathrm{rr}}^{(1), 0,[8]}\right] \\
& -\frac{1}{8}\left(\operatorname{Re}\left[\widehat{H}_{\mathrm{rr}}^{(1), 0,[8]]}\right)^{2}+\frac{1}{2} \operatorname{Re}\left[\widehat{H}_{\mathrm{rr}}^{(2), 0,[8]}\right],\right.
\end{aligned}
$$

with $\mathrm{r}=q, g$. We use this definition to compute quark and gluon impact factors at two loops and report their singularities

$$
C_{q}^{(2)}=\frac{1}{8 \varepsilon^{4}} C_{F}^{2}+\frac{1}{\varepsilon^{3}}\left(\frac{17}{64} N_{c}^{2}-\frac{23}{64}-\frac{1}{16} n_{f} C_{F}+\frac{3}{32} \frac{1}{N_{c}^{2}}\right)
$$




$$
\begin{aligned}
&+ \frac{1}{\varepsilon^{2}}\left[N_{c}^{2}\left(\frac{31}{384}-\frac{3}{16} \zeta(2)\right)+\frac{7}{32} \zeta(2)-\frac{77}{192}+\frac{1}{24} n_{f} C_{F}+\frac{1}{N_{c}^{2}}\left(\frac{41}{128}-\frac{1}{32} \zeta(2)\right)\right] \\
&+\frac{1}{\varepsilon}\left[N_{c}^{2}\left(-\frac{1037}{2304}-\frac{19}{48} \zeta(2)+\frac{1}{96} \zeta(3)\right)-\frac{119}{288}+\frac{19}{48} \zeta(2)+\frac{31}{96} \zeta(3)\right. \\
&\left.\quad+\left(\frac{1}{24} \zeta(2)+\frac{89}{288}\right) n_{f} C_{F}+\frac{1}{N_{c}^{2}}\left(\frac{221}{256}-\frac{1}{3} \zeta(3)\right)\right]+\mathcal{O}\left(\varepsilon^{0}\right), \\
& C_{g}^{(2)}=\frac{1}{8 \varepsilon^{4}} N_{c}^{2}+\frac{7}{32 \varepsilon^{3}} b_{0} N_{c}+\frac{1}{\varepsilon^{2}}\left[N_{c}^{2}\left(\frac{103}{96}-\frac{7}{16} \zeta(2)\right)-\frac{49}{144} n_{f} N_{c}+\frac{1}{36} n_{f}^{2}\right] \\
&+\frac{1}{\varepsilon}\left[N_{c}^{2}\left(\frac{853}{864}-\frac{11}{12} \zeta(2)-\frac{31}{48} \zeta(3)\right)+n_{f} N_{c}\left(-\frac{67}{288}+\frac{1}{6} \zeta(2)\right)\right. \\
&\left.\quad+\frac{5}{216} n_{f}^{2}-\frac{1}{32} \frac{n_{f}}{N_{c}}\right]+\mathcal{O}\left(\varepsilon^{0}\right),
\end{aligned}
$$

where we have adopted for simplicity a mixed notation, writing explicitly $C_{F}$ instead of $\left(N_{c}^{2}-1\right) /\left(2 N_{c}\right)$ and $b_{0}$ instead of $\left(11 N_{c}-2 n_{f}\right) / 3$ whenever such factors appear explicitly in the calculation.

Similarly, the remainder functions $R_{\mathrm{rs}}^{(2), 0,[8]}$ are written somewhat formally in eq. (4.32), but they can be easily made explicit, for each parton species, upon picking specific color bases for the various amplitudes. Working in the orthonormal bases described in detail in appendix $\mathrm{A}$ and in refs. [22, 23], we get, for the octet components of quark and gluon amplitudes,

$$
\begin{aligned}
& R_{q q}^{(2), 0,[8]}=\frac{\pi^{2}}{4 \epsilon^{2}}\left(1-\frac{3}{N_{c}^{2}}\right)\left(1-\epsilon^{2} \zeta(2)\right), \\
& R_{g g}^{(2), 0,[8]}=-\frac{3 \pi^{2}}{2 \epsilon^{2}}\left(1-\epsilon^{2} \zeta(2)\right) \\
& R_{q g}^{(2), 0,[8]}=-\frac{\pi^{2}}{4 \epsilon^{2}}\left(1-\epsilon^{2} \zeta(2)\right) .
\end{aligned}
$$

Notice that our remainder at this order has no contributions of order $\varepsilon^{-1}$, as a consequence of the structure of infrared factorization. Furthermore, the factors $\left(1-\epsilon^{2} \zeta(2)\right)$ can all be absorbed in the constant $c_{\Gamma}^{2}$, by performing the expansion in terms of $\tilde{\alpha}_{s}=\alpha_{s} c_{\Gamma}$, instead of using $\alpha_{s}$ : in this scheme, the octet remainder is a pure double pole. This explains, as we will see shortly, the result of ref. [4], where a violation of universality with only a double-pole contribution was discovered at the two-loop level.

We finally consider our proposed expression for the impact factors, eq. (4.33). It contains terms which are manifestly universal and consistent with the interpretation of $C_{\mathrm{r}}$ : for example, the first line of eq. (4.33) naturally arises from the action of the exponential jet factors $Z_{1, \mathbf{R}, \mathrm{rr}}$, as defined in eq. (2.11), on the hard factors, and can be unambiguously assigned to the external legs of the amplitude. Armed with these results and definitions, we can now check that our corrected high-energy factorization formula, eq. (3.1), works, by comparing the exact $q g \rightarrow q g$ amplitude at two loops, taken from ref. [18], with the one constructed using eq. (3.1), with the impact factors, the Regge trajectory, and the remainder functions defined above. We find, as expected, that $R_{q g}^{(2), 0,[8]}$, together with the impact factors defined in eq. (4.33), accounts for the complete two-loop quark-gluon scattering amplitude 
in the high-energy limit, including finite parts. Note that the same consistency check was performed in section 4.1 on the $q g$ amplitude at one loop: in that case, universality was recovered by means of both eq. (4.16), derived from the definition of $\mathcal{Z}_{1, \mathbf{R}, \mathrm{rr}}$, and eq. (4.17), which is of the same form but is required by high-energy factorization. Similarly, an important ingredient for universality of eq. (3.1) at two loops is the two-loop jet factor identity

$$
Z_{1, \mathbf{R}, q g}^{(2)}=\frac{1}{8}\left[4 Z_{1, \mathbf{R}, q q}^{(2)}+4 Z_{1, \mathbf{R}, g g}^{(2)}+2 Z_{1, \mathbf{R}, q q}^{(1)} Z_{1, \mathbf{R}, g g}^{(1)}-\left(Z_{1, \mathbf{R}, q q}^{(1)}\right)^{2}-\left(Z_{1, \mathbf{R}, g g}^{(1)}\right)^{2}\right],
$$

which is a simple consequence of eq. (2.11) and of the exponential form of the color singlet functions $\mathcal{Z}_{1, \mathbf{R}}$. On the other hand, the consistency check on the $q g \rightarrow q g$ scattering amplitude implies that an identity of the same form must hold for the finite parts too. This can be verified directly using the results of ref. [18]. One finds that

$$
\begin{aligned}
\operatorname{Re}\left[\widehat{H}_{q g}^{(2), 0,[8]}\right]= & \frac{1}{8}\left[4 \operatorname{Re}\left[\widehat{H}_{q q}^{(2), 0,[8]}\right]+4 \operatorname{Re}\left[\widehat{H}_{g g}^{(2), 0,[8]}\right]+2 \operatorname{Re}\left[\widehat{H}_{q q}^{(1), 0,[8]}\right] \operatorname{Re}\left[\widehat{H}_{g g}^{(1), 0,[8]}\right]\right. \\
& \left.-\left(\operatorname{Re}\left[\widehat{H}_{q q}^{(1), 0,[8]}\right]\right)^{2}-\left(\operatorname{Re}\left[\widehat{H}_{g g}^{(1), 0,[8]}\right]\right)^{2}\right]
\end{aligned}
$$

The structure of eq. (4.37), as well as that of eq. (4.17), suggest a simple exponential ansatz for the impact factors, involving the jet factors of eq. (2.11) and the non-logarithmic terms of the hard functions. To see it, we define the functions

$$
\widehat{H}_{\mathbf{R}, \mathrm{rs}}^{0,[8]}=\sum_{n=0}^{\infty}\left(\frac{\alpha_{s}}{\pi}\right)^{n} \operatorname{Re}\left[\widehat{H}_{\mathrm{rs}}^{(n), 0,[8]}\right],
$$

and we simply assume that they exponentiate just like the jet factors $Z_{1, \mathbf{R}, \text { rs }}$ in eq. (2.11). We can then write

$$
Z_{1, \mathbf{R}, \mathrm{rs}}=\exp \left[\zeta_{\mathrm{r}}\right] \times \exp \left[\zeta_{\mathrm{s}}\right], \quad \widehat{H}_{\mathbf{R}, \mathrm{rs}}^{0,[8]}=\exp \left[h_{\mathrm{r}}\right] \times \exp \left[h_{\mathrm{s}}\right],
$$

where the functions $h$ and $\zeta$ have perturbative expansions

$$
h_{\mathrm{r}}\left(\alpha_{s}\right)=\frac{\alpha_{s}}{\pi} h_{\mathrm{r}}^{(1)}+\left(\frac{\alpha_{s}}{\pi}\right)^{2} h_{\mathrm{r}}^{(2)}+\ldots, \quad \zeta_{\mathrm{r}}\left(\alpha_{s}\right)=\frac{\alpha_{s}}{\pi} \zeta_{\mathrm{r}}^{(1)}+\left(\frac{\alpha_{s}}{\pi}\right)^{2} \zeta_{\mathrm{r}}^{(2)}+\ldots
$$

By using this notation, we can express the coefficients of the perturbative expansions of $Z_{1, \mathbf{R}, \mathrm{rs}}$ and $\widehat{H}_{\mathrm{rs}}^{(n), 0,[8]}$ simply as

$$
\begin{aligned}
Z_{1, \mathbf{R}, \mathrm{rs}}^{(1)} & =\frac{1}{2}\left(\zeta_{\mathrm{r}}^{(1)}+\zeta_{\mathrm{s}}^{(1)}\right), \\
Z_{1, \mathbf{R}, \mathrm{rs}}^{(2)} & =\frac{1}{2}\left(\zeta_{\mathrm{r}}^{(2)}+\zeta_{\mathrm{s}}^{(2)}\right)+\frac{1}{2}\left(\frac{\zeta_{\mathrm{r}}^{(1)}+\zeta_{\mathrm{s}}^{(1)}}{2}\right)^{2}, \\
\operatorname{Re}\left[\widehat{H}_{\mathrm{rs}}^{(1), 0,[8]]}\right. & =\frac{1}{2}\left(h_{\mathrm{r}}^{(1)}+h_{\mathrm{s}}^{(1)}\right), \\
\operatorname{Re}\left[\widehat{H}_{\mathrm{rs}}^{(2), 0,[8]}\right] & =\frac{1}{2}\left(h_{\mathrm{r}}^{(2)}+h_{\mathrm{s}}^{(2)}\right)+\frac{1}{2}\left(\frac{h_{\mathrm{r}}^{(1)}+h_{\mathrm{s}}^{(1)}}{2}\right)^{2} .
\end{aligned}
$$


We are now in a position to rewrite the definitions of the impact factors at one and two loops, eq. (4.14) and eq. (4.33), using eq. (4.41). We obtain simply

$$
\begin{aligned}
C_{\mathrm{r}}^{(1)} & =\frac{\zeta_{\mathrm{r}}^{(1)}+h_{\mathrm{r}}^{(1)}}{2}, \\
C_{\mathrm{r}}^{(2)} & =\frac{\zeta_{\mathrm{r}}^{(2)}+h_{\mathrm{r}}^{(2)}}{2}+\frac{1}{2}\left(\frac{\zeta_{\mathrm{r}}^{(1)}+h_{\mathrm{r}}^{(1)}}{2}\right)^{2} .
\end{aligned}
$$

This suggests a formal definition of impact factors to all orders, based on the information we get from infrared factorization, and on the properties of the hard functions up to two loops. We write

$$
C_{\mathrm{r}}=\exp \left[\frac{\zeta_{\mathrm{r}}+h_{\mathrm{r}}}{2}\right]
$$

which is exact at two loops with our definition of impact factor, and can be conjectured to provide a consistent definition to all orders. Intriguingly, eq. (4.43) involves the exponentiation of non-logarithmic, finite contributions to the amplitude: similar effects have been known for a long time [29, 58-61] for form factors and cross sections that are electroweak at tree level: eq. (4.43) provides a hint that this kind of exponentiation might extend to multi-particle amplitudes, at least in the high-energy limit.

We conclude the discussion at the two-loop level by noting that we are now in a position to recover the violation of universality first diagnosed in ref. [4], where the authors were assuming that high-energy factorization would work without a remainder function. Under that assumption, one finds a discrepancy between the exact two-loop quark-gluon scattering amplitude and the one predicted by the high-energy factorization formula, eq. (3.1), in the absence of the remainder $R$. That mismatch may be quantified by the function [11]

$$
\begin{aligned}
\Delta_{(2), 0,[8]} & =\frac{M_{q g}^{(2), 0}}{H_{q g}^{(0),[8]}}-\left[C_{q}^{(2)}+C_{g}^{(2)}+C_{q}^{(1)} C_{g}^{(1)}-\frac{\pi^{2}}{4}(1+\kappa)\left(\alpha^{(1)}\right)^{2}\right] \\
& =\frac{1}{2}\left[R_{q g}^{(2), 0,[8]}-\frac{1}{2}\left(R_{q q}^{(2), 0,[8]}+R_{g g}^{(2), 0,[8]}\right)\right] .
\end{aligned}
$$

Using eq. (4.32) and eq. (4.35), we may evaluate explicitly eq. (4.44), finding

$$
\begin{aligned}
\Delta_{(2), 0,[8]} & =\frac{3}{2} \pi^{2}\left(K^{(1)}\right)^{2}\left(\frac{N_{c}^{2}+1}{N_{c}^{2}}\right)\left(1-\epsilon^{2} \zeta(2)\right) \\
& =\frac{\pi^{2}}{\varepsilon^{2}} \frac{3}{16}\left(\frac{N_{c}^{2}+1}{N_{c}^{2}}\right)\left(1-\epsilon^{2} \zeta(2)\right) .
\end{aligned}
$$

Up to our different normalization, already discussed above eq. (2.8), and noting again that our expansion parameter is $\alpha_{s}$ and not $\tilde{\alpha}_{s}$, one easily sees that eq. (4.45) is in complete agreement with ref. [4], and explains the origin of the problem, as arising from the mixing of color representations induced by infrared factorization.

\subsection{Three-loop matrix elements}

Proceeding to three-loop order, we expect that matching the single-logarithmic terms in eqs. (3.1) and (2.2) will lead to a breaking of universality similar to that observed for nonlogarithmic terms at two loops. Indeed, as predicted in refs. [6, 7], a direct comparison 
yields a non-universal result. As before, we begin by expanding eq. (2.2) to third order in $\alpha_{s}$. We obtain

$$
\begin{aligned}
& M^{(3), 0}=\left[\mathrm { i } \frac { \pi ^ { 3 } } { 4 8 } ( K ^ { ( 1 ) } ) ^ { 3 } \left(-\mathbf{O}_{s-u}^{3}+8 \mathbf{O}_{s, t, s}\left(1+\kappa_{\mathrm{rs}}\right)-6 \mathbf{O}_{s-u} \mathbf{O}_{t, s}\left(1+\kappa_{\mathrm{rs}}\right)\right.\right. \\
& +2 \mathbf{O}_{t, t, s}\left(1+\kappa_{\mathrm{rs}}\right)^{2}-3 \mathbf{O}_{t}^{2} \mathbf{O}_{s-u}\left(1+\kappa_{\mathrm{rs}}\right)^{2} \\
& \left.+\mathbf{O}_{t}^{3}\left(1+\kappa_{\mathrm{rs}}\right)^{3}+3 \mathbf{O}_{t} \mathbf{O}_{s-u}^{2}\left(1+\kappa_{\mathrm{rs}}\right)+6 \mathbf{O}_{t} \mathbf{O}_{t, s}\left(1+\kappa_{\mathrm{rs}}\right)^{2}\right) \\
& +\frac{\pi^{2}}{8} K^{(1)}\left(K^{(1)} Z_{1, \mathbf{R}}^{(1)}+2 K^{(2)}\right)\left(-\mathbf{O}_{s-u}^{2}-2 \mathbf{O}_{t, s}\left(1+\kappa_{\mathrm{rs}}\right)\right. \\
& \left.+2 \mathbf{O}_{t} \mathbf{O}_{s-u}\left(1+\kappa_{\mathrm{rs}}\right)-\mathbf{O}_{t}^{2}\left(1+\kappa_{\mathrm{rs}}\right)^{2}\right) \\
& \left.+\mathrm{i} \frac{\pi}{2}\left(K^{(1)} Z_{1, \mathbf{R}}^{(2)}+K^{(2)} Z_{1, \mathbf{R}}^{(1)}+K^{(3)}\right)\left(\mathbf{O}_{s-u}-\mathbf{O}_{t}\left(1+\kappa_{\mathrm{rs}}\right)\right)+Z_{1, \mathbf{R}}^{(3)}\right] H^{(0)} \\
& +\left[\frac { \pi ^ { 2 } } { 8 } ( K ^ { ( 1 ) } ) ^ { 2 } \left(-\mathbf{O}_{s-u}^{2}-2 \mathbf{O}_{t, s}\left(1+\kappa_{\mathrm{rs}}\right)\right.\right. \\
& \left.+2 \mathbf{O}_{t} \mathbf{O}_{s-u}\left(1+\kappa_{\mathrm{rs}}\right)-\mathbf{O}_{t}^{2}\left(1+\kappa_{\mathrm{rs}}\right)^{2}\right) \\
& \left.+\mathrm{i} \frac{\pi}{2}\left(K^{(1)} Z_{1, \mathbf{R}}^{(1)}+K^{(2)}\right)\left(\mathbf{O}_{s-u}-\mathbf{O}_{t}\left(1+\kappa_{\mathrm{rs}}\right)\right)+Z_{1, \mathbf{R}}^{(2)}\right] H^{(1), 0} \\
& +\left[\mathrm{i} \frac{\pi}{2} K^{(1)}\left(\mathbf{O}_{s-u}-\mathbf{O}_{t}\left(1+\kappa_{\mathrm{rs}}\right)\right)+Z_{1, \mathbf{R}}^{(1)}\right] H^{(2), 0}+H^{(3), 0}, \\
& M^{(3), 1}=\left[\frac { \pi ^ { 2 } } { 2 4 } ( K ^ { ( 1 ) } ) ^ { 3 } \left(-8 \mathbf{O}_{s, t, s}+6 \mathbf{O}_{s-u} \mathbf{O}_{t, s}+6 \mathbf{O}_{t}^{2} \mathbf{O}_{s-u}\left(1+\kappa_{\mathrm{rs}}\right)\right.\right. \\
& \left.-3 \mathbf{O}_{t}^{3}\left(1+\kappa_{\mathrm{rs}}\right)^{2}-3 \mathbf{O}_{t} \mathbf{O}_{s-u}^{2}-12 \mathbf{O}_{t} \mathbf{O}_{t, s}\left(1+\kappa_{\mathrm{rs}}\right)\right) \\
& +\mathrm{i} \frac{\pi}{2} K^{(1)}\left(K^{(1)} Z_{1, \mathbf{R}}^{(1)}+2 K^{(2)}\right)\left(-\mathbf{O}_{t, s}+\mathbf{O}_{t} \mathbf{O}_{s-u}-\mathbf{O}_{t}^{2}\left(1+\kappa_{\mathrm{rs}}\right)\right) \\
& \left.+\left(K^{(3)}+K^{(2)} Z_{1, \mathbf{R}}^{(1)}+K^{(1)} Z_{1, \mathbf{R}}^{(2)}\right) \mathbf{O}_{t}\right] H^{(0)} \\
& +\left[\mathrm{i} \frac{\pi}{2}\left(K^{(1)}\right)^{2}\left(-\mathbf{O}_{t, s}+\mathbf{O}_{t} \mathbf{O}_{s-u}-\mathbf{O}_{t}^{2}\left(1+\kappa_{\mathrm{rs}}\right)\right)+\left(K^{(1)} Z_{1, \mathbf{R}}^{(1)}+K^{(2)}\right) \mathbf{O}_{t}\right] H^{(1), 0} \\
& +\left[\frac { \pi ^ { 2 } } { 8 } ( K ^ { ( 1 ) } ) ^ { 2 } \left(-\mathbf{O}_{s-u}^{2}-2 \mathbf{O}_{t, s}\left(1+\kappa_{\mathrm{rs}}\right)\right.\right. \\
& \left.+2 \mathbf{O}_{t} \mathbf{O}_{s-u}\left(1+\kappa_{\mathrm{rs}}\right)-\mathbf{O}_{t}^{2}\left(1+\kappa_{\mathrm{rs}}\right)^{2}\right) \\
& \left.+\mathrm{i} \frac{\pi}{2}\left(K^{(1)} Z_{1, \mathbf{R}}^{(1)}+K^{(2)}\right)\left(\mathbf{O}_{s-u}-\mathbf{O}_{t}\left(1+\kappa_{\mathrm{rs}}\right)\right)+Z_{1, \mathbf{R}}^{(2)}\right] H^{(1), 1} \\
& +K^{(1)} \mathbf{O}_{t} H^{(2), 0}+\left[\mathrm{i} \frac{\pi}{2} K^{(1)}\left(\mathbf{O}_{s-u}-\mathbf{O}_{t}\left(1+\kappa_{\mathrm{rs}}\right)\right)+Z_{1, \mathbf{R}}^{(1)}\right] H^{(2), 1}+H^{(3), 1}, \\
& M^{(3), 2}=\left[\mathrm{i} \frac{\pi}{12}\left(K^{(1)}\right)^{3}\left(2 \mathbf{O}_{t, t, s}-6 \mathbf{O}_{t} \mathbf{O}_{t, s}+3 \mathbf{O}_{t}^{2} \mathbf{O}_{s-u}-3 \mathbf{O}_{t}^{3}\left(1+\kappa_{\mathrm{rs}}\right)\right)\right. \\
& \left.+\frac{1}{2} K^{(1)}\left(K^{(1)} Z_{1, \mathbf{R}}^{(1)}+2 K^{(2)}\right) \mathbf{O}_{t}^{2}\right] H^{(0)}+\frac{1}{2}\left(K^{(1)}\right)^{2} \mathbf{O}_{t}^{2} H^{(1), 0}
\end{aligned}
$$




$$
\begin{aligned}
+ & {\left[\mathrm{i} \frac{\pi}{2}\left(K^{(1)}\right)^{2}\left(-\mathbf{O}_{t, s}+\mathbf{O}_{t} \mathbf{O}_{s-u}-\mathbf{O}_{t}^{2}\left(1+\kappa_{\mathrm{rs}}\right)\right)\right.} \\
+ & \left.\left(K^{(2)}+K^{(1)} Z_{1, \mathbf{R}}^{(1)}\right) \mathbf{O}_{t}\right] H^{(1), 1}+K^{(1)} \mathbf{O}_{t} H^{(2), 1} \\
+ & {\left[\mathrm{i} \frac{\pi}{2} K^{(1)}\left(\mathbf{O}_{s-u}-\mathbf{O}_{t}\left(1+\kappa_{\mathrm{rs}}\right)\right)+Z_{1, \mathbf{R}}^{(1)}\right] H^{(2), 2}+H^{(3), 2} } \\
M^{(3), 3}= & \frac{\left(K^{(1)}\right)^{3}}{6} \mathbf{O}_{t}^{2} H^{(0)}+\frac{\left(K^{(1)}\right)^{2}}{2} \mathbf{O}_{t}^{2} H^{(1), 1}+K^{(1)} \mathbf{O}_{t} H^{(2), 2}+H^{(3), 3},
\end{aligned}
$$

where each of eqs. (4.46)-(4.49) is a vector in color space. For the octet component, expanding eq. (3.1) to third order in $\alpha_{s}$ we find

$$
\begin{aligned}
& M_{\mathrm{rs}}^{(3), 0,[8]}=\left\{C_{\mathrm{r}}^{(3)}+C_{\mathrm{s}}^{(3)}+C_{\mathrm{r}}^{(1)} C_{\mathrm{s}}^{(2)}+C_{\mathrm{r}}^{(2)} C_{\mathrm{s}}^{(1)}\right. \\
& -\frac{\pi^{2}}{4}\left(\alpha^{(1)}\right)^{2}\left(C_{\mathrm{r}}^{(1)}+C_{\mathrm{s}}^{(1)}\right)\left(1+\kappa_{\mathrm{rs}}\right)-\frac{\pi^{2}}{2} \alpha^{(1)} \alpha^{(2)}\left(1+\kappa_{\mathrm{rs}}\right) \\
& +\mathrm{i} \pi\left[\left(\frac{\pi^{2}}{12}\left(\alpha^{(1)}\right)^{3}-\frac{\alpha^{(2)}}{2}\left(C_{\mathrm{r}}^{(1)}+C_{\mathrm{s}}^{(1)}\right)-\frac{\alpha^{(3)}}{2}\right)\left(1+\kappa_{\mathrm{rs}}\right)\right. \\
& \left.\left.-\frac{\alpha^{(1)}}{2}\left(1+\kappa_{\mathrm{rs}}\right)\left(C_{\mathrm{r}}^{(2)}+C_{\mathrm{s}}^{(2)}+C_{\mathrm{r}}^{(1)} C_{\mathrm{s}}^{(1)}\right)\right]\right\} H_{\mathrm{rs}}^{(0),[8]}+\frac{R^{(3), 0,[8]}}{2} H_{\mathrm{rs}}^{(0)}, \\
& M_{\mathrm{rs}}^{(3), 1,[8]}=\left[\alpha^{(3)}+\alpha^{(2)}\left(C_{\mathrm{r}}^{(1)}+C_{\mathrm{s}}^{(1)}\right)+\alpha^{(1)}\left(C_{\mathrm{r}}^{(1)} C_{\mathrm{s}}^{(1)}+C_{\mathrm{r}}^{(2)}+C_{\mathrm{s}}^{(2)}\right)\right. \\
& -\frac{\pi^{2}}{4}\left(\alpha^{(1)}\right)^{3}\left(1+\kappa_{\mathrm{rs}}\right) \\
& \left.-\mathrm{i} \pi\left(1+\kappa_{\mathrm{rs}}\right)\left(\frac{\left(\alpha^{(1)}\right)^{2}}{2}\left(C_{\mathrm{r}}^{(1)}+C_{\mathrm{s}}^{(1)}\right)+\alpha^{(1)} \alpha^{(2)}\right)\right] H_{\mathrm{rs}}^{(0),[8]}+\frac{R^{(3), 1,[8]}}{2} H_{\mathrm{rs}}^{(0)}, \\
& M_{\mathrm{rs}}^{(3), 2,[8]}=\left[\frac{\left(\alpha^{(1)}\right)^{2}}{2}\left(C_{\mathrm{r}}^{(1)}+C_{\mathrm{s}}^{(1)}\right)+\alpha^{(1)} \alpha^{(2)}-\mathrm{i} \pi \frac{\left(\alpha^{(1)}\right)^{3}}{4}\left(1+\kappa_{\mathrm{rs}}\right)\right] H_{\mathrm{rs}}^{(0),[8]} \text {, } \\
& M_{\mathrm{rs}}^{(3), 3,[8]}=\frac{\left(\alpha^{(1)}\right)^{3}}{6} H_{\mathrm{rs}}^{(0),[8]} \text {. }
\end{aligned}
$$

where in the first two equations we allow for a non-factorizing remainder, as in eq. (4.3). Notice that the Reggeization of next-to-leading logarithms was proven in ref. [2] only for the real part of the scattering amplitude, therefore in principle we should allow for a nonvanishing purely imaginary remainder $R^{(3), 2,[8]}$. We have seen at two loops, however, that eq. (3.1) yields the correct result for the imaginary part of the octet amplitude not only at NLL level, but in fact even at NNLL. Furthermore we note that IR factorization, as seen for example in eq. (3.5), does not generate any contribution at NLL for the octet component of the amplitude, thanks to the identity in eq. (3.11). We conjecture therefore that eq. (3.1) yields the exact result for the octet component of the amplitude at NLL level, both for the real and for the imaginary part, and we set $R^{(n), n-1,[8]}=0$. 
With this premise, we can proceed as we did at two loops. We start by comparing the coefficients of the leading logarithms, that is eq. (4.49) and the bottom expression of eq. (4.50), and we verify that the coefficient of the highest power of the energy logarithm is determined by the one-loop result. In particular, the imaginary part of the hard matrix element vanishes, while the real part is of higher order in $\varepsilon$,

$$
\begin{aligned}
\operatorname{Im}\left[H_{\mathrm{rs}}^{(3), 3,[8]}\right] & =0 \\
\operatorname{Re}\left[H_{\mathrm{rs}}^{(3), 3,[8]}\right] & =\frac{1}{6} \frac{\operatorname{Re}\left[H_{\mathrm{rs}}^{(1), 1,[8]}\right]^{3}}{\left(H_{\mathrm{rs}}^{(0),[8]}\right)^{2}}=\mathcal{O}\left(\varepsilon^{3}\right) .
\end{aligned}
$$

In order to inspect the NLL terms, we compare eq. (4.48) with the next-to-last expression in eq. (4.50). Considering first the imaginary part, and using our assumption that eq. (3.1) works up to NLL for the octet amplitude, we expect to find relations allowing us to express the NLL finite parts $H_{\mathrm{rs}}^{(3), 2,[8]}$ in terms of lower-loop amplitudes. A direct comparison yields a somewhat unwieldy expression

$$
\begin{aligned}
\operatorname{Im}\left[H_{\mathrm{rs}}^{(3), 2,[8]]}=\right. & -\frac{\pi}{12}\left(K^{(1)}\right)^{3}\left[\left(2 \mathbf{O}_{t, t, s}-6 \mathbf{O}_{t} \mathbf{O}_{t, s}+3 \mathbf{O}_{t}^{2} \mathbf{O}_{s-u}-3 \mathbf{O}_{t}^{3}\left(1+\kappa_{\mathrm{rs}}\right)\right) H_{\mathrm{rs}}^{(0)}\right]^{[8]} \\
& -\frac{\pi}{2}\left(K^{(1)}\right)^{2}\left[\left(-\mathbf{O}_{t, s}+\mathbf{O}_{t} \mathbf{O}_{s-u}-\mathbf{O}_{t}^{2}\left(1+\kappa_{\mathrm{rs}}\right)\right) \operatorname{Re}\left(H_{\mathrm{rs}}^{(1), 1}\right)\right]^{[8]} \\
& -\left(K^{(2)}+K^{(1)} Z_{1, \mathbf{R}}^{(1)}\right)\left[\mathbf{O}_{t} \operatorname{Im}\left(H_{\mathrm{rs}}^{(1), 1}\right)\right]^{[8]}-K^{(1)}\left[\mathbf{O}_{t} \operatorname{Im}\left(H_{\mathrm{rs}}^{(2), 1}\right)\right]^{[8]} \\
& -\frac{\pi}{2} K^{(1)}\left[\left(\mathbf{O}_{s-u}-\mathbf{O}_{t}\left(1+\kappa_{\mathrm{rs}}\right)\right) \operatorname{Re}\left(H_{\mathrm{rs}}^{(2), 2}\right)\right]^{[8]}-Z_{1, \mathbf{R}}^{(1)} \operatorname{Im}\left[H_{\mathrm{rs}}^{(2), 2,[8]}\right] \\
& -\frac{1}{2}\left(K^{(1)}\right)^{2}\left[\mathbf{O}_{t}^{2} \operatorname{Im}\left(H_{\mathrm{rs}}^{(1), 0}\right)\right]^{[8]}-\frac{\pi}{4}\left(\alpha^{(1)}\right)^{3}\left(1+\kappa_{\mathrm{rs}}\right) H_{\mathrm{rs}}^{(0),[8]} .
\end{aligned}
$$

The right-hand side of eq. (4.52) can however be simplified considerably, by making use of the fact that all color-mixing operators appearing in eq. (4.52) give zero when applied either on the tree level amplitude $H^{(0),[8]}$, or on the leading-logarithmic finite parts $\operatorname{Re}\left[H^{(1), 1}\right]$, $\operatorname{Re}\left[H^{(2), 2}\right]$, whose only non-vanishing component is the octet amplitude. Furthermore, one can use the identities in eqs. (4.20), (4.13), (4.8) and (4.9) to express the corresponding finite parts of the amplitude in terms of lower order quantities, or set them to zero. In this way, we find that

$$
\operatorname{Im}\left[H_{\mathrm{rs}}^{(3), 2,[8]}\right]=-\frac{\pi}{4}\left(1+\kappa_{\mathrm{rs}}\right)\left[\widehat{H}_{\mathrm{rs}}^{(1), 1,[8]}\right]^{3} H_{\mathrm{rs}}^{(0),[8]}=O\left(\varepsilon^{3}\right) .
$$

Performing the same procedure on the real part, we find

$$
\operatorname{Re}\left[H_{\mathrm{rs}}^{(3), 2,[8]}\right]=\operatorname{Re}\left[H_{\mathrm{rs}}^{(2), 1,[8]}\right] \widehat{H}_{\mathrm{rs}}^{(1), 1,[8]}-\frac{1}{2} \operatorname{Re}\left[H_{\mathrm{rs}}^{(1), 0,[8]}\right]\left(\widehat{H}_{\mathrm{rs}}^{(1), 1,[8]}\right)^{2}=\mathcal{O}(\epsilon) .
$$

As was the case at two loops, we predict that octet hard parts actually vanish in $d=4$ up to NLL.

Proceeding to the NNLL terms, it is interesting to inspect first the imaginary part of $M_{\mathrm{rs}}^{(3), 1,[8]}$, and briefly explore the possibility that also at three loops the imaginary part of 
the NNLL octet remainder function might vanish, as it does at two loops. In that case, equating the second expressions in eqs. (4.49) and (4.50), we obtain a lengthy expression which can be drastically simplified using the same techniques that led to eq. (4.53). The result is

$$
\begin{aligned}
\operatorname{Im}\left[H_{\mathrm{rs}}^{(3), 1,[8]}\right]= & \frac{\pi}{2}\left(K^{(1)}\right)^{2}\left(\mathbf{O}_{t, s}-\mathbf{O}_{t} \mathbf{O}_{s-u}\right) \operatorname{Re}\left[H_{\mathrm{rs}}^{(1), 0}\right]-\frac{\pi}{2} K^{(1)} \mathbf{O}_{s-u} \operatorname{Re}\left[H_{\mathrm{rs}}^{(2), 1}\right] \\
& -\pi\left(1+\kappa_{\mathrm{rs}}\right) \operatorname{Re}\left[H_{\mathrm{rs}}^{(3), 2,[8]}\right] .
\end{aligned}
$$

The first line involves color-mixing operators acting on $\operatorname{Re}\left[H^{(1), 0}\right], \operatorname{Re}\left[H^{(2), 1}\right]$. The effect of these operators is to generate contributions to the non-octet components of the amplitude. The information available about those terms can be extracted from the calculation of the two-loop amplitudes for parton-parton scattering of refs. [17-19], whose high-energy limit can be found in appendix C. Both terms vanish to the highest available order in $\epsilon$, i.e., $\operatorname{Re}\left[H^{(1), 0,[k]}\right]=0$ through $O\left(\epsilon^{2}\right)$, and $\operatorname{Re}\left[H_{\mathrm{rs}}^{(2), 1,[k]}\right]=0$ through $O\left(\epsilon^{0}\right)$ for $k \neq 8$. As a consequence, we can conclude that the first term in the first line of eq. (4.55) is at least $\mathcal{O}(\varepsilon)$, while the second is $\mathcal{O}\left(\varepsilon^{0}\right)$. It is interesting to note that, if the uncalculated $\mathcal{O}(\epsilon)$ terms in $\operatorname{Re}\left[H_{\mathrm{rs}}^{(2), 1,[k]}\right]=0$ turn out to vanish, then, at least in the $\epsilon \rightarrow 0$ limit, one would find the simplified expression

$$
\operatorname{Im}\left[H_{\mathrm{rs}}^{(3), 1,[8]}\right]=-\pi\left(1+\kappa_{\mathrm{rs}}\right) \operatorname{Re}\left[H_{\mathrm{rs}}^{(3), 2,[8]}\right],
$$

which is strongly reminiscent of the NNLL imaginary part at two loops, eq. (4.27).

We finally proceed to the real part of $M_{\mathrm{rs}}^{(3), 1,[8]}$ at NNLL accuracy, which is the level at which the three-loop Regge trajectory shows up. Inspecting eq. (4.47), we can easily single out potential contributions to the remainder function $R^{(3), 1,[8]}$, by looking for the color mixing operators which give a non-zero result when acting on the color octet amplitude. It is however clear that at three loops we will not be able to give a complete expression for $R^{(3), 1[8]}$, since single-logarithmic finite contributions can arise directly in $H^{(3)}$, which is unknown. Singular single-logarithmic terms at three loops are however completely predicted, under our assumption that the dipole formula applies at this order, since all relevant anomalous dimensions are known, and finite contributions to the amplitude are known up to two loops.

With this in mind, we start our inspection of $M^{(3), 1}$ in eq. (4.47) by identifying the terms involving the operators $\mathbf{O}_{s, t, s}, \mathbf{O}_{s-u} \mathbf{O}_{t, s}$ and $\mathbf{O}_{t} \mathbf{O}_{s-u}^{2}$ in the first two lines as contributions to the octet remainder. These terms multiply $\left(K^{(1)}\right)^{3} H^{(0)}$, and thus are $\mathcal{O}\left(\varepsilon^{-3}\right)$, which is consistent with the fact that the NNLL octet remainder at two loops is $\mathcal{O}\left(\varepsilon^{-2}\right)$. Additional contributions arise from the operator $\mathbf{O}_{s-u}^{2}$ applied to $H^{(1), 1}: H^{(1), 1}$ is $\mathcal{O}(\varepsilon)$, but it is multiplied by $\left(K^{(1)}\right)^{2}$, so this term is $\mathcal{O}\left(\varepsilon^{-1}\right)$. Proceeding further, we see that terms which involve $H^{(0)}$ or $H^{(1), 1}$ and are linear in operators like $\mathbf{O}_{s-u}$ and $\mathbf{O}_{t, s}$ do not contribute to the octet remainder: in fact, $H^{(0)}$ and $H^{(1), 1}$ are pure octets, and therefore they give vectors with a vanishing octet component when acted upon by these color-mixing operators. This reasoning however does not work when we consider terms involving $H^{(1), 0}$ : in fact, in this case $\operatorname{Im}\left[H^{(1), 0,[k]}\right]$ with $k \neq 8$ is different from zero; thus, the operators $\mathbf{O}_{t, s}$ 
and $\mathbf{O}_{t} \mathbf{O}_{s-u}$, acting on $H^{(1), 0}$, will contribute to the remainder. Likewise, contributions could arise from the operator $\mathbf{O}_{s-u}$ acting on $\operatorname{Im}\left[H^{(2), 1,[k]}\right]$, with $k \neq 8$, in the last line of eq. (4.47). These contributions however will not give any poles, since $\operatorname{Im}\left(H^{(2), 1,[k]}\right)=0$, for any $k \neq 8$, as can be seen by using the explicit results given in appendix $\mathrm{C}$.

Further contributions to the octet remainder arise from terms involving the color diagonal operator $\mathbf{O}_{t}=\mathbf{T}_{t}^{2}$, because of a mismatch between the structure predicted by eq. (3.1) and the terms originating from $\mathbf{O}_{t}$ in eq. (3.5), similarly to what we observed at two loops. In the case of $M^{(3), 1}$, these are the terms involving the operators $\mathbf{O}_{t}^{3}\left(1+\kappa_{\mathrm{rs}}\right)^{2}$ and $\mathbf{O}_{t}^{2}\left(1+\kappa_{\mathrm{rs}}\right)^{2}$. The mismatch occurs because the factor $\left(1+\kappa_{\mathrm{rs}}\right)$ appears only linearly in the corresponding terms of $M^{(3), 1}$ in eq. (4.50), after the explicit values of the Regge trajectory and the impact factors are inserted. The two terms above are $\mathcal{O}\left(\varepsilon^{-3}\right)$ and $\mathcal{O}\left(\varepsilon^{-1}\right)$ respectively. Proceeding further, one could pin down further contributions to the octet remainder at $\mathcal{O}\left(\varepsilon^{0}\right)$, but those are beyond the reach of the present analysis, as explained previously.

We are now in a position to give an expression for the octet remainder at three loops, $R^{(3), 1,[8]}$, where we collect all terms through $\mathcal{O}\left(\varepsilon^{-1}\right)$. We find

$$
\begin{aligned}
R_{\mathrm{rs}}^{(3), 1,[8]}= & \frac{\pi^{2}}{4}\left(K^{(1)}\right)^{3}\left[\left(-\frac{8}{3} \mathbf{O}_{s, t, s}+2 \mathbf{O}_{s-u} \mathbf{O}_{t, s}-\mathbf{O}_{t} \mathbf{O}_{s-u}^{2}+\mathbf{O}_{t}^{3}\left(1-\kappa_{\mathrm{rs}}^{2}\right)\right) \widehat{H}_{\mathrm{rs}}^{(0)}\right]^{[8]} \\
+ & \left(K^{(1)}\right)^{2}\left[\pi\left(\mathbf{O}_{t, s}-\mathbf{O}_{t} \mathbf{O}_{s-u}\right) \operatorname{Im}\left[\widehat{H}_{\mathrm{rs}}^{(1), 0}\right]-\frac{\pi^{2}}{4} \mathbf{O}_{s-u}^{2} \operatorname{Re}\left[\widehat{H}_{\mathrm{rs}}^{(1), 1}\right]\right. \\
& \left.+\frac{3}{4} \pi^{2} \mathbf{O}_{t}^{2}\left(1-\kappa_{\mathrm{rs}}^{2}\right) \operatorname{Re}\left[\widehat{H}_{\mathrm{rs}}^{(1), 1}\right]\right]^{[8]}+\mathcal{O}\left(\epsilon^{0}\right)
\end{aligned}
$$

With this definition, it is easy to verify that the divergent part of the three-loop Regge trajectory retains a universal form. One finds, as expected

$$
\alpha^{(3)}=K^{(3)} N_{c}+\mathcal{O}\left(\epsilon^{0}\right)
$$

Introducing in eq. (4.57) the appropriate color factors and hard functions, and working in the color bases discussed in the appendix A, we obtain the explicit results

$$
\begin{aligned}
& R_{q q}^{(3), 1,[8]}=\left(\frac{\alpha_{s}}{\pi}\right)^{3} \frac{\pi^{2}}{\epsilon^{3}} \frac{2 N_{c}^{2}-5}{12 N_{c}}\left(1-\frac{3}{2} \varepsilon^{2} \zeta(2)\right)+\mathcal{O}\left(\varepsilon^{0}\right) \\
& R_{g g}^{(3), 1,[8]}=-\left(\frac{\alpha_{s}}{\pi}\right)^{3} \frac{\pi^{2}}{\epsilon^{3}} \frac{2}{3} N_{c}\left(1-\frac{3}{2} \varepsilon^{2} \zeta(2)\right)+\mathcal{O}\left(\varepsilon^{0}\right) \\
& R_{q g}^{(3), 1,[8]}=-\left(\frac{\alpha_{s}}{\pi}\right)^{3} \frac{\pi^{2}}{\epsilon^{3}} \frac{N_{c}}{24}\left(1-\frac{3}{2} \varepsilon^{2} \zeta(2)\right)+\mathcal{O}\left(\varepsilon^{0}\right)
\end{aligned}
$$

which can be consistently used in eq. (3.1), provided one substitutes the impact factors and the Regge trajectory as defined in eqs. (4.33) and (4.58). Once again, remarkably, we find that the singular parts of the remainders originate from a high-order pole (here $\epsilon^{-3}$ ), with lower order poles arising exclusively from the expansion of the constant $c_{\Gamma}^{3}$ : expanding in powers of $\tilde{\alpha}_{s}$, the singular parts of the three-loop octet remainders are pure triple poles.

Comparing $M^{(3), 0}$ in eqs. (4.49) and (4.50), one could single out contributions to the octet remainder $R^{(3), 0,[8]}$, which would be necessary to obtain a consistent definition of the 
impact factors $C_{\mathrm{rs}}^{(3)}$ to $\mathrm{N}^{3} \mathrm{LL}$ accuracy, through $\mathcal{O}\left(\varepsilon^{-1}\right)$. That analysis is straightforward but lengthy, and since it does not provide additional insight in the mechanism of breaking of eq. (3.1), we will not perform it in this work. We conclude by noting that, if a nonvanishing quadrupole contribution to the soft anomalous dimension were to be discovered, it would affect eq. (4.57) and eq. (4.59) at the level of single poles.

\subsection{Beyond three loops}

As we have seen, by using the information provided by infrared factorization, we are able to pin down the origin of the breakdown of eq. (3.1) at NNLL accuracy, and define a remainder function which collects non-universal terms. Since infrared factorization correctly reproduces the infrared poles of an amplitude, but gives no prediction for its finite parts, we are able to extend this procedure only up to terms which involve finite parts which are known through explicit calculations. On the other hand, the high-energy factorization embodied in eq. (3.1) is exact up to NLL accuracy for real parts of amplitudes, ${ }^{3}$ and, we assume, for octet imaginary parts as well. This enables us to give NLL predictions concerning finite parts of amplitudes as well, to all orders in perturbation theory.

Let us start by inspecting leading logarithmic terms. First we note that, at LL accuracy, the infrared factorization formula, eq. (2.2), can be written as

$$
\begin{aligned}
\mathcal{M}_{\mathrm{LL}}^{[8]} & \left(\frac{s}{\mu^{2}}, \frac{t}{\mu^{2}}, \alpha_{s}\right)=\left[\left(\frac{s}{-t}\right)^{K\left(\alpha_{s}\right) \mathbf{O}_{t}} \mathcal{H}_{\mathrm{LL}}\right]^{[8]} \\
& =4 \pi \alpha_{s} H^{(0),[8]} \sum_{n=0}^{\infty}\left(\frac{\alpha_{s}}{\pi}\right)^{n} \log ^{n}\left(\frac{s}{-t}\right)\left(\sum_{k=0}^{n} \frac{N_{c}^{k}}{k !}\left(K^{(1)}\right)^{k} \operatorname{Re}\left[\widehat{H}^{(n-k), n-k,[8]}\right]\right)
\end{aligned}
$$

while in case of the Regge factorization formula we can write

$$
\begin{aligned}
\mathcal{M}_{\mathrm{rs}, \mathrm{LL}}^{[8]}\left(\frac{s}{\mu^{2}}, \frac{t}{\mu^{2}}, \alpha_{s}\right) & =2 \pi \alpha_{s} H_{\mathrm{rs}}^{(0),[8]}\left[\left(\frac{s}{-t}\right)^{\alpha(t)}\left[1+\mathrm{e}^{-\mathrm{i} \pi \alpha(t)}+\kappa_{\mathrm{rs}}\left(1-\mathrm{e}^{-\mathrm{i} \pi \alpha(t)}\right)\right]\right]_{\mathrm{LL}} \\
& =4 \pi \alpha_{s} H_{\mathrm{rs}}^{(0),[8]} \sum_{n=0}^{\infty}\left(\frac{\alpha_{s}}{\pi}\right)^{n} \frac{\left(\alpha^{(1)}(t)\right)^{n}}{n !} \log ^{n}\left(\frac{s}{-t}\right)
\end{aligned}
$$

Using the explicit result for $\alpha^{(1)}(t)$, and comparing term by term eq. (4.60) with eq. (4.61), it is easy to find that

$$
\begin{aligned}
\operatorname{Im}\left[H^{(n), n}\right] & =0 \\
\operatorname{Re}\left[H^{(n), n,[8]}\right] & =\frac{1}{n !}\left(\operatorname{Re}\left[H^{(1), 1,[8]}\right]\right)^{n}=\mathcal{O}\left(\epsilon^{n}\right) .
\end{aligned}
$$

Interestingly, and extending to all orders the results obtained up to three loops, LL hard parts vanish in $d=4$, as a consequence of the fact that the one-loop Regge trajectory is essentially a pure pole in dimensional regularization. A finite contribution to $\alpha^{(1)}$ would in fact spoil eq. (4.62).

\footnotetext{
${ }^{3}$ Specifically, we note that we are assuming here that high-energy factorization holds at NLL also for $\mathcal{O}(\varepsilon)$ terms, which are not known at two loops.
} 
With a little more work, this result generalizes to NLL. At this accuracy, the infrared factorization formula in eq. (2.2) yields

$$
\begin{aligned}
\mathcal{M}_{\mathrm{NLL}}^{[8]}\left(\frac{s}{\mu^{2}}, \frac{t}{\mu^{2}}, \alpha_{s}\right)=\left[\mathcal{Z}_{\mathrm{NLL}}\right. & \left(\frac{s}{\mu^{2}}, \frac{t}{\mu^{2}}, \alpha_{s}\right) \mathcal{H}_{\mathrm{LL}}\left(\frac{s}{\mu^{2}}, \frac{t}{\mu^{2}}, \alpha_{s}\right) \\
& \left.+\mathcal{Z}_{\mathrm{R}, \mathrm{LL}}\left(\frac{s}{t}, \alpha_{s}\right) \mathcal{H}_{\mathrm{NLL}}\left(\frac{s}{\mu^{2}}, \frac{t}{\mu^{2}}, \alpha_{s}\right)\right]^{[8]} .
\end{aligned}
$$

The second term can easily be written down explicitly. It is given by

$$
\begin{aligned}
{\left[\mathcal{Z}_{\mathrm{R}, \mathrm{LL}}\left(\frac{s}{t}, \alpha_{s}\right) \mathcal{H}_{\mathrm{NLL}}\left(\frac{s}{\mu^{2}}, \frac{t}{\mu^{2}}, \alpha_{s}\right)\right]^{[8]}=} & 4 \pi \alpha_{s} H_{\mathrm{rs}}^{(0),[8]} \sum_{n=1}^{\infty}\left(\frac{\alpha_{s}}{\pi}\right)^{n} \log ^{n-1}\left(\frac{s}{-t}\right) \\
& \sum_{\sigma=0}^{n-1} \frac{\left(K^{(1)} N_{c}\right)^{n-\sigma-1}}{(n-\sigma-1) !} \widehat{H}_{\mathrm{rs}}^{(\sigma+1), \sigma,[8]} .
\end{aligned}
$$

The first term in eq. (4.63), on the other hand, can be significantly simplified by noting that the only non-vanishing component of the vector $\mathcal{H}_{\mathrm{LL}}$ is the color octet, and therefore the color octet component of the result is annihilated by the operators $\mathbf{O}_{s-u}$ and $\mathbf{O}_{t, \ldots, t, s}$ appearing in $\mathcal{Z}_{\mathrm{NLL}}$. One obtains then

$$
\begin{aligned}
{\left[\mathcal{Z}_{\mathrm{NLL}}\right.} & \left.\left(\frac{s}{\mu^{2}}, \frac{t}{\mu^{2}}, \alpha_{s}\right) \mathcal{H}_{\mathrm{LL}}\left(\frac{s}{\mu^{2}}, \frac{t}{\mu^{2}}, \alpha_{s}\right)\right]^{[8]}=\sum_{n=1}^{\infty}\left(\frac{\alpha_{s}}{\pi}\right)^{n} \log ^{n-1}\left(\frac{s}{-t}\right) \\
& \times\left\{\sum_{\sigma=0}^{n-1} \frac{\left(K^{(1)} N_{c}\right)^{n-\sigma-1}}{(n-\sigma-1) !}\left[\mathcal{Z}_{\mathbf{R}}^{(1)}-\mathrm{i} \pi K^{(1)} \frac{\left(1+\kappa_{\mathrm{rs}}\right)}{2} N_{c}\right] \widehat{H}_{\mathrm{rs}}^{(\sigma), \sigma,[8]}\right. \\
& \left.+\sum_{\sigma=0}^{n-2} \frac{\left(K^{(1)} N_{c}\right)^{n-\sigma-2}}{(n-\sigma-2) !} K^{(2)} N_{c} \widehat{H}_{\mathrm{rs}}^{(\sigma), \sigma,[8]}\right\} .
\end{aligned}
$$

The NLL prediction from the Regge factorization formula, eq. (3.1), gives instead

$$
\begin{aligned}
\mathcal{M}_{\mathrm{rs}, \mathrm{NLL}}^{[8]}=4 \pi \alpha_{s} H_{\mathrm{rs}}^{(0),[8]} \sum_{n=1}^{\infty}\left(\frac{\alpha_{s}}{\pi}\right)^{n} & {\left[\left(C_{\mathrm{r}}^{(1)}+C_{\mathrm{s}}^{(1)}\right) \frac{\left(\alpha^{(1)}\right)^{n-1}}{(n-1) !}+\alpha^{(2)} \frac{\left(\alpha^{(1)}\right)^{n-2}}{(n-2) !}\right.} \\
& \left.-\mathrm{i} \frac{\pi}{2}\left(1+\kappa_{\mathrm{rs}}\right) \frac{\left(\alpha^{(1)}\right)^{n}}{n !}\right] \log ^{n-1}\left(\frac{s}{-t}\right)
\end{aligned}
$$

Under our assumption that eq. (3.1) is correct up to NLL also for the imaginary part of the octet component of the amplitude, we can use the fact that the Regge trajectory and the impact factors are real. We can then substitute their explicit values, as obtained in eqs. (4.11) and (4.14), and proceed to compare eq. (4.66) with the sum of eqs. (4.64) and (4.65). We get

$$
\begin{aligned}
\operatorname{Im}\left[\widehat{H}_{\mathrm{rs}}^{(n), n-1,[8]}\right] & =-\pi \frac{1+\kappa_{\mathrm{rs}}}{2} n \widehat{H}^{(n), n,[8]}=O\left(\epsilon^{n}\right), \\
\operatorname{Re}\left[\widehat{H}^{(n), n-1,[8]}\right] & =\operatorname{Re}\left[\widehat{H}^{(2), 1,[8]}\right] \widehat{H}^{(n-2), n-2,[8]}+(2-n) \operatorname{Re}\left[\widehat{H}^{(1), 0,[8]}\right] \widehat{H}^{(n-1), n-1,[8]} \\
& =\mathcal{O}\left(\epsilon^{n-2}\right), \quad \text { for } n>2 .
\end{aligned}
$$


Also at NLL, we find that hard coefficients at each loop order are determined in terms of the coefficients at lower orders. Furthermore we find that both real and imaginary parts of the hard function vanish in $d=4$, except $\operatorname{Re}\left[\widehat{H}^{(1), 0,[8]}\right]$ and $\operatorname{Re}\left[\widehat{H}^{(2), 1,[8]}\right]$ which cannot be determined by eq. (4.67). In essence, eq. (4.67) reinforces the idea that high-energy logarithms are infrared in nature: indeed, leading and next-to-leading logarithmic contributions to hard scattering coefficients are forced to vanish with increasing powers of the regulator $\varepsilon$. This means that infrared-finite high-energy logarithms must come exclusively from the interference of soft and collinear functions with lower-order contributions subleading in $\varepsilon$.

\section{On subleading color amplitudes}

The central idea at the basis of our analysis in section 4 is that the interplay of high-energy and infrared factorization allows one to obtain more information about the leading coloroctet amplitude than would be allowed by inspection of the two factorization formulae separately. Taken individually, the two factorizations have different limitations: infrared factorization predicts all infrared poles of the amplitude, but does not control finite parts. High-energy factorization, on the other hand, predicts both poles and finite parts of the color octet amplitude, once the Regge trajectory and impact factors are known, but the predictions have a limited logarithmic accuracy. Combining the two factorizations, on the one hand one can use infrared information to determine the poles of the remainder function at NNLL and beyond: this results in consistent definitions of the two-loop impact factors, eq. (4.33), and of the three-loop Regge trajectory, eq. (4.58). On the other hand, highenergy factorization allows one to derive, to all orders, the NLL part of the color-octet hard functions appearing in the infrared factorization formula. The knowledge of these terms, in turn, gives access to a set of higher-order contributions to the amplitude, not only for color octet exchange, but also for other representations contributing to the scattering process. In this section we briefly explore the predictions that can be obtained in this way.

In order to be more explicit, let us start with an example. The state of the art QCD computations provide us with one-loop amplitudes to all order in $\varepsilon$, and two-loop amplitudes up to $\mathcal{O}\left(\varepsilon^{0}\right)$, for $2 \rightarrow 2$ processes in all partonic channels, $q q \rightarrow q q, g g \rightarrow g g$ and $q g \rightarrow q g$. Organising this knowledge in terms of the dipole formula allows us to extract the one-loop and two-loop hard functions, whose high-energy limit is given in appendix C, respectively up to $\mathcal{O}\left(\varepsilon^{2}\right)$ and $\mathcal{O}\left(\varepsilon^{0}\right)$. With this knowledge at hand, one can generically predict the corresponding amplitudes at three loops up to $\mathcal{O}\left(\varepsilon^{-2}\right)$, at four loops up to $\mathcal{O}\left(\varepsilon^{-4}\right)$, and so on. If, however, one inputs information from high-energy factorization, such as eq. (4.67), we can extend the prediction to lower-order poles in $\varepsilon$, for the coefficients of leading and next-to-leading logarithms. The pole structure of the leading logarithms is simple: for gluon-gluon scattering we have

$$
\begin{aligned}
M_{g g}^{(3), 3,[1]} & =M_{g g}^{(3), 3,\left[8_{s}\right]}=M_{g g}^{(3), 3,[10+\overline{10}]}=M_{g g}^{(3), 3,[27]}=M_{g g}^{(3), 3,[0]}=0, \\
M_{g g}^{(3), 3,\left[8_{a}\right]} & =-\frac{N_{c}^{4} \sqrt{N_{c}^{2}-1}}{24 \epsilon^{3}} \frac{s}{t}\left(1-\frac{3}{2} \varepsilon^{2} \zeta(2)\right)+\mathcal{O}\left(\epsilon^{0}\right)
\end{aligned}
$$


where, once again, the terms in bracket simply recover the expansion of $c_{\Gamma}$. The quarkquark amplitude gives

$$
\begin{aligned}
& M_{q q}^{(3), 3,[1]}=0 \\
& M_{q q}^{(3), 3,[8]}=\frac{N_{c}^{3} \sqrt{N_{c}^{2}-1}}{24 \epsilon^{3}} \frac{s}{t}\left(1-\frac{3}{2} \varepsilon^{2} \zeta(2)\right)+\mathcal{O}\left(\epsilon^{0}\right) .
\end{aligned}
$$

Finally, for quark-gluon scattering,

$$
\begin{aligned}
M_{q g}^{(3), 3,[1]} & =M_{q g}^{(3), 3,\left[8_{s}\right]}=0, \\
M_{q g}^{(3), 3,\left[8_{a}\right]} & =\frac{N_{c}^{3} \sqrt{N_{c}\left(N_{c}^{2}-1\right)}}{24 \sqrt{2} \epsilon^{3}} \frac{s}{t}\left(1-\frac{3}{2} \varepsilon^{2} \zeta(2)\right)+\mathcal{O}\left(\epsilon^{0}\right) .
\end{aligned}
$$

We can similarly obtain the poles of NLL amplitudes. For instance, using eq. (4.48), we realize that, since $H^{(2), 2}=\mathcal{O}\left(\varepsilon^{2}\right)$ and $H^{(3), 3}=\mathcal{O}\left(\varepsilon^{3}\right), M^{(3), 3,[8]}$ can be predicted up to $\mathcal{O}\left(\varepsilon^{0}\right)$, and $M^{(3), 2,[8]}$ can be predicted up to $\mathcal{O}\left(\varepsilon^{-1}\right)$. We find

$$
\begin{aligned}
M_{g g}^{(3), 2,[8]}= & N_{c}^{3} \sqrt{N_{c}^{2}-1} \frac{s}{t}\left\{\frac{1}{4 \epsilon^{4}}+\frac{11 N_{c}-2 n_{f}+\mathrm{i} \pi N_{c}}{16 \epsilon^{3}}-\frac{5}{8} N_{c} \zeta(2) \frac{1}{\epsilon^{2}}\right. \\
& \left.+\frac{1}{\epsilon}\left[-\frac{3}{16} b_{0} \zeta(2)-\frac{7}{4} N_{c} \zeta(3)-\mathrm{i} \pi \frac{3}{32} N_{c} \zeta(2)\right]\right\}+\mathcal{O}\left(\epsilon^{0}\right) .
\end{aligned}
$$

As regards the quark-quark and quark-gluon scattering processes, we find respectively

$$
\begin{aligned}
M_{q q}^{(3), 2,[8]}= & N_{c} \sqrt{N_{c}^{2}-1} \frac{s}{t}\left\{\frac{1}{\epsilon^{4}}\left(-\frac{N_{c}^{2}-1}{16}\right)+\frac{1}{\epsilon^{3}}\left(-\frac{5}{24} N_{c}^{2}+\frac{1}{48} n_{f} N_{c}+\frac{3}{32}-\frac{\mathrm{i} \pi}{8}\right)\right. \\
+ & \frac{1}{\epsilon^{2}}\left[N_{c}^{2}\left(\frac{7}{32} \zeta(2)+\frac{5}{18}\right)-\frac{5}{72} n_{f} N_{c}+\frac{1}{4}-\frac{3}{32} \zeta(2)\right] \\
+ & \frac{1}{\epsilon}\left[N_{c}^{2}\left(\frac{121}{216}+\frac{9}{64} \zeta(2)+\frac{7}{16} \zeta(3)\right)\right. \\
& \left.\left.\left.-\frac{7}{54} n_{f} N_{c}+\frac{1}{2}-\frac{9}{64} \zeta(2)-\frac{7}{16} \zeta(3)+\mathrm{i} \pi \frac{3}{16} \zeta(2)\right)\right]\right\}+\mathcal{O}\left(\epsilon^{0}\right),
\end{aligned}
$$

and

$$
\begin{aligned}
& M_{q g}^{(3), 2,[8]}= \frac{\sqrt{N_{c}^{3}\left(N_{c}^{2}-1\right)}}{\sqrt{2}} \frac{s}{t}\left\{\frac{1}{\epsilon^{4}}\left(\frac{1-3 N_{c}^{2}}{16}\right)+\frac{1}{\epsilon^{3}}\left(-\frac{53}{96} N_{c}^{2}+\frac{n_{f} N_{c}}{12}+\frac{3}{32}-\mathrm{i} \pi \frac{N_{c}^{2}}{16}\right)\right. \\
&+\frac{1}{\epsilon^{2}}\left[N_{c}^{2}\left(\frac{5}{18}+\frac{17}{32} \zeta(2)\right)-\frac{5}{72} n_{f} N_{c}+\frac{1}{4}-\frac{3}{32} \zeta(2)\right] \\
&+\frac{1}{\epsilon}\left[N_{c}^{2}\left(\frac{121}{216}+\frac{31}{64} \zeta(2)+\frac{21}{16} \zeta(3)+\mathrm{i} \pi \frac{3}{32} \zeta(2)\right)\right. \\
&\left.\left.\left.-n_{f} N_{c}\left(\frac{7}{54}+\frac{1}{16} \zeta(2)\right)+\frac{1}{2}-\frac{9}{64} \zeta(2)-\frac{7}{16} \zeta(3)\right)\right]\right\}+\mathcal{O}\left(\epsilon^{0}\right) .
\end{aligned}
$$

We next ask to what extent we can predict other color components of the amplitudes, which are subleading in the high-energy limit. The dipole formula applies to the vector amplitude in color space, therefore of course we are able to obtain predictions for the infrared 
poles of subleading color amplitudes as well. These amplitudes however do not admit a high-energy factorization formula of the form of eq. (3.1). This can be easily understood inspecting eq. (3.4): non-octet amplitudes vanish at tree level in the high-energy limit, and are generated at one loop because of the effect of the operator $\mathbf{O}_{s-u}$, acting on the octet component. In the language of Regge theory, these contributions are associated with cuts in the complex angular momentum plane, as opposed to the leading color-octet amplitude, which can be described at least up to NLL in terms of angular momentum poles only. Contributions arising from Regge cuts are expected to obey their own form of Regge factorization, different from eq. (3.1): a proposal in this direction was put forward in ref. [16], where a formula for the even-even color-subleading amplitudes at NLL was provided, leading in particular to the prediction that the dipole formula must receive corrections at NLL and at the four-loop order. For comparison and future reference, we provide here a set of predictions at three and four loops for the poles of color-subleading amplitudes associated with leading and next-to-leading logarithms, which we derive from the dipole formula in the absence of corrections. The starting point is the vanishing of the one- and two-loop LL and NLL hard parts for the non-octet amplitudes, which can be seen from the explicit result in appedix C, and is in agreement with ref. [16]. Specifically, we find that

$$
\begin{gathered}
H_{\mathrm{rs}}^{(n), n,[i \neq 8]}=0 \quad(n=1,2), \\
\operatorname{Re}\left[H_{\mathrm{rs}}^{(1), 0,[i \neq 8]]}=0, \quad \operatorname{Im}\left[H_{\mathrm{rs}}^{(1), 0,[i \neq 8]}\right]=\mathcal{O}(\varepsilon), \quad H_{\mathrm{rs}}^{(2), 1,[i \neq 8]}=\mathcal{O}(\varepsilon) .\right.
\end{gathered}
$$

Using this information, and inspecting eq. (4.48), we see that all components of four-parton amplitudes in the high-energy limit can be fully predicted at NLL, up to $\mathcal{O}\left(\varepsilon^{-1}\right)$. The results are, for the three-loop gluon-gluon amplitude,

$$
\begin{aligned}
M_{g g}^{(3), 2,[1]} & =\mathrm{i} \pi \frac{N_{c}^{4}}{12 \epsilon^{3}} \frac{s}{t}\left(1-\frac{3}{2} \epsilon^{2} \zeta(2)\right)+\mathcal{O}\left(\epsilon^{0}\right), \\
M_{g g}^{(3), 2,\left[8_{s}\right]} & =\mathrm{i} \pi \frac{N_{c}^{4} \sqrt{N_{c}^{2}-1} \frac{s}{16 \epsilon^{3}}\left(1-\frac{3}{2} \epsilon^{2} \zeta(2)\right)+\mathcal{O}\left(\epsilon^{0}\right),}{t} M_{g g}^{(3), 2,[10+\overline{10}]}=\mathcal{O}\left(\epsilon^{0}\right), \\
M_{g g}^{(3), 2,[27]} & =\mathrm{i} \pi \frac{N_{c} \sqrt{\left(N_{c}+3\right)\left(N_{c}-1\right)}}{24 \epsilon^{3}} \frac{s}{t}\left(7 N_{c}^{2}+10 N_{c}+4\right)\left(1-\frac{3}{2} \epsilon^{2} \zeta(2)\right)+\mathcal{O}\left(\epsilon^{0}\right), \\
M_{g g}^{(3), 2,[0]} & =\mathrm{i} \pi \frac{N_{c} \sqrt{\left(N_{c}-3\right)\left(N_{c}+1\right)}}{24 \epsilon^{3}} \frac{s}{t}\left(7 N_{c}^{2}-10 N_{c}+4\right)\left(1-\frac{3}{2} \epsilon^{2} \zeta(2)\right)+\mathcal{O}\left(\epsilon^{0}\right) .
\end{aligned}
$$

For the three-loop quark-quark amplitude we find

$$
M_{q q}^{(3), 2,[1]}=\mathrm{i} \pi \frac{N_{c}\left(N_{c}^{2}-1\right)}{48 \epsilon^{3}} \frac{s}{t}\left(1-\frac{3}{2} \epsilon^{2} \zeta(2)\right)+\mathcal{O}\left(\epsilon^{0}\right)
$$

Finally, for the quark gluon amplitude we find

$$
\begin{aligned}
M_{q g}^{(3), 2,[1]} & =-\mathrm{i} \pi \frac{N_{c}^{2} \sqrt{N_{c}\left(N_{c}^{2}-1\right)}}{24 \epsilon^{3}} \frac{s}{t}\left(1-\frac{3}{2} \epsilon^{2} \zeta(2)\right)+\mathcal{O}\left(\epsilon^{0}\right) \\
M_{q g}^{(3), 2,\left[8_{s}\right]} & =-\mathrm{i} \pi \frac{N_{c}^{2} \sqrt{N_{c}\left(N_{c}^{2}-1\right)\left(N_{c}^{2}-4\right)}}{16 \sqrt{2} \epsilon^{3}} \frac{s}{t}\left(1-\frac{3}{2} \epsilon^{2} \zeta(2)\right)+\mathcal{O}\left(\epsilon^{0}\right) .
\end{aligned}
$$


Also in the case of eqs. (5.8), (5.9), and (5.10), singular terms are given by just triple poles if one expands in powers of $\tilde{\alpha}_{s}$.

To complete this section, we go one order higher and consider the four-loop expression for the amplitude, as predicted by the dipole formula. We provide the amplitude up to NLL in $\log (-s / t)$, because we know that constraints from Regge factorization arise only up to this order. We find

$$
\begin{aligned}
M^{(4), 4}= & \frac{\left(K^{(1)}\right)^{4}}{24} \mathbf{O}_{t}^{4} H^{(0)}+\frac{\left(K^{(1)}\right)^{3}}{6} \mathbf{O}_{t}^{3} H^{(1), 1}+\frac{\left(K^{(1)}\right)^{2}}{2} \mathbf{O}_{t}^{2} H^{(2), 2} \\
& +\mathbf{O}_{t} K^{(1)} H^{(3), 3}+H^{(4), 4} \\
M^{(4), 3}= & \left\{\mathrm{i} \frac{\pi}{24}\left(K^{(1)}\right)^{4}\left[-\mathbf{O}_{t, t, t, s}+4 \mathbf{O}_{t} \mathbf{O}_{t, t, s}-6 \mathbf{O}_{t}^{2} \mathbf{O}_{t, s}+2 \mathbf{O}_{t}^{3} \mathbf{O}_{s-u}-2 \mathbf{O}_{t}^{4}\left(1+\kappa_{\mathrm{rs}}\right)\right]\right. \\
& \left.+\frac{\left(K^{(1)}\right)^{3}}{6} Z_{1, \mathbf{R}}^{(1)} \mathbf{O}_{t}^{3}+\frac{\left(K^{(1)}\right)^{2} K^{(2)}}{2} \mathbf{O}_{t}^{3}\right\} H^{(0)}+\frac{\left(K^{(1)}\right)^{3}}{6} \mathbf{O}_{t}^{3} H^{(1), 0} \\
+ & \left\{\mathrm{i} \frac{\pi}{12}\left(K^{(1)}\right)^{3}\left[2 \mathbf{O}_{t, t, s}-6 \mathbf{O}_{t} \mathbf{O}_{t, s}+3 \mathbf{O}_{t}^{2} \mathbf{O}_{s-u}-3 \mathbf{O}_{t}^{3}\left(1+\kappa_{\mathrm{rs}}\right)\right]\right. \\
& \left.+K^{(1)} K^{(2)} \mathbf{O}_{t}^{2}+\frac{\left(K^{(1)}\right)^{2}}{2} Z_{1, \mathbf{R}}^{(1)} \mathbf{O}_{t}^{2}\right\} H^{(1), 1}+\frac{\left(K^{(1)}\right)^{2}}{2} \mathbf{O}_{t}^{2} H^{(2), 1} \\
+ & \left\{\mathrm{i} \frac{\pi}{2}\left(K^{(1)}\right)^{2}\left[-\mathbf{O}_{t, s}+\mathbf{O}_{t} \mathbf{O}_{s-u}-\mathbf{O}_{t}^{2}\left(1+\kappa_{\mathrm{rs}}\right)\right]+K^{(2)} \mathbf{O}_{t}+K^{(1)} Z_{\mathbf{1}, \mathbf{R}}^{(1)} \mathbf{O}_{t}\right\} H^{(2), 2} \\
+ & K^{(1)} \mathbf{O}_{t} H^{(3), 2}+\mathrm{i} \frac{\pi}{2} K^{(1)}\left[\mathbf{O}_{s-u}-\mathbf{O}_{t}\left(1+\kappa_{\mathrm{rs}}\right)\right] H^{(3), 3}+Z_{\mathbf{1 , R}}^{(1)} H^{(3), 3}+H^{(4), 3} .
\end{aligned}
$$

Taking into account eqs. (4.67) and (5.7), as well as the results in appendix B and appendix C, one can compute the LL amplitudes $M^{(4), 4}$ up to $\mathcal{O}\left(\varepsilon^{-1}\right)$, and the NLL amplitudes $M^{(4), 3}$ up to $\mathcal{O}\left(\varepsilon^{-2}\right)$. Indeed, inspecting eq. (5.11), we see that one would need the knowledge of $H^{(2), 1,[i \neq 8]}$ up to $\mathcal{O}(\varepsilon)$ in order to obtain $M^{(4), 3}$ up to $\mathcal{O}\left(\varepsilon^{-1}\right)$. We will not display here the corresponding lengthy expressions, but they can readily be obtained combining eq. (5.11) with the results given in the appendices.

Here we will focus instead on the color singlet amplitude, which is a bit special, because $\mathbf{O}_{t}^{2} H^{(2), 1,[1]}=0$. This enables us to compute $M^{(4), 3,[1]}$ up to $\mathcal{O}\left(\varepsilon^{-1}\right)$. The result is particularly interesting in light of the recent claim [16] that this term receives a contribution not predicted by the dipole formula. Within our setup we can provide a partial check, in the form an independent prediction of the $\varepsilon^{-1}$ poles arising within the infrared factorization by using the dipole formula only.

We start by noting that the terms proportional to $\mathbf{O}_{t} \mathbf{O}_{t, s}, \mathbf{O}_{t}^{2} \mathbf{O}_{s-u}$ and $\mathbf{O}_{t}$ in eq. (5.11) have a vanishing color-singlet component. The only source of $\varepsilon^{-1}$ poles in $M^{(4), 3,[1]}$ is then the term proportional to $\left(K^{(1)}\right)^{3} \mathbf{O}_{t, t, s}$. One finds

$$
\left.M_{g g}^{(4), 3,[1]}\right|_{\varepsilon^{-1}}=\frac{N_{c}^{5}}{12} \frac{7}{6 \epsilon} \mathrm{i} \pi \zeta_{3} \frac{s}{t} .
$$


As expected from ref. [16], it can be verified that eq. (5.12) comes entirely from the expansion of the common loop factor $c_{\Gamma}$ in eq. (4.5), and it can therefore be reabsorbed in the corresponding redefinition of the coupling. We confirm then that, when expanded in terms of the high-energy coupling $\tilde{\alpha}_{s}$, the dipole formula does not generate simple poles at NLL for color-singlet $t$-channel exchange, and the non-vanishing result found in ref. [16] must be considered as a violation of the dipole formula at the four-loop level.

\section{Conclusion}

The high-energy limit, $s /|t| \rightarrow \infty$, of gauge theory amplitudes is of great theoretical and phenomenological interest, and has been a major focus of investigation for several decades. The range of applications is vast: on the one hand, the high-energy limit can be used to study formal properties of scattering amplitudes in highly symmetric gauge theories, such as $N=4$ Super Yang-Mills theory (see, for example, refs. [63, 64] for recent applications in this direction); on the other hand, it is very relevant for phenomenological applications to cross sections of interest at colliders such as LHC (see, as recent examples, refs. [65, 66]).

The main reason beyond this wide range of applications is the simplicity of high-energy amplitudes. As $s /|t| \rightarrow \infty$, amplitudes come to be dominated by logarithmic enhancements, which can be studied to all orders in perturbation theory with the tool of Gribov-Regge theory, as well as with the more typical tools of perturbative QCD. In the case of four parton amplitudes, at leading and next-to-leading logarithmic accuracy, an all-order factorization holds, which resums energy logarithms based on the fact that, to this accuracy, the only relevant singularities in the complex $J$ plane are simple poles.

It is generally understood [3], however, and in fact it has been proven [2], that this simple Reggeization picture of QCD scattering amplitudes cannot be generally applicable beyond NLL accuracy. It is also understood that a complete Reggeization picture should include the contributions of Regge cuts at sufficiently high orders in perturbation theory, possibly beginning with non-planar contributions to scattering amplitudes at the threeloop level [5]. The details of how those cuts arise, and thus of how the simple Reggeization picture breaks down, are however not known.

Building upon the earlier analyses of refs. [6-10], which examined the interplay of high-energy factorization and infrared factorization, in ref. [11] we outlined a roadmap to explore the violations of the simple Reggeization picture, and thus the rise of the Regge cuts, by comparing the two factorizations, when applied to QCD scattering amplitudes order by order in perturbation theory. The immediate outcome was to explain the origin of a non-factorizing term, independent of $\ln (s /|t|)$, first uncovered in ref. [4] in amplitudes for parton-parton scattering at the two-loop level. In addition, since it was already clear $[6,7]$ that the presence of non-factorizing terms in $\ln (s /|t|)$ at three-loops would invalidate the notion of a universal Regge trajectory, in ref. [11] we proposed a scheme to gather non-universal contributions into a non-factorizing remainder function, and we used infrared factorization to re-define the impact factors and the Regge trajectory as functions of universal terms only. Those definitions allow one in principle to compute the complete three-loop Regge trajectory unambiguously. 
In this paper, we have provided the details of the roadmap sketched in ref. [11], and we have presented a complete analysis of four-parton QCD scattering amplitudes in the highenergy limit, including all available results up to three loops, and deriving some all-order relations. In particular, we have performed a detailed comparison of infrared and high-energy factorizations up to three-loop order, for both real and imaginary parts of the amplitudes. The cross-fertilization between the two approaches yields a number of interesting results.

First of all, infrared factorization allowed us to identify non-universal terms affecting Regge behavior, and therefore to predict the infrared poles of the factorization-breaking terms up to three loops. To that accuracy, it is now possible to define unambiguously impact factors, the Regge trajectory, and the remainder functions. In addition, we analysed the $t$ channel exchange of color representations other than the octet in scattering amplitudes up to three-loops, at leading and next-to-leading logarithmic accuracy, and, as an example, we computed the infrared pole of a single-logarithmic term at four loops, in the singlet component of gluon-gluon scattering, at next-to-leading logarithmic accuracy. The fourloop NLL single-pole contribution to singlet exchange arising from the dipole formula can be reabsorbed in the definition of the coupling: any contribution without this property must thus be considered as a violation of the dipole formula at four loops. Therefore we agree that the findings of ref. [16] imply a violation of the dipole formula at this perturbative order.

On the other hand, high-energy factorization at LL and NLL level provides all-order constraints on the hard functions defined by infrared factorization. Under mild and wellmotivated assumptions, that high-energy factorization should extend to the NLL imaginary part of $t$-channel octet exchange, and to $\mathcal{O}(\varepsilon)$ contributions to the amplitude, we have derived a set of all-order identities showing that all hard functions for four-parton scattering amplitudes in QCD vanish in $d=4$ in the high-energy limit, up to NLL accuracy. This result considerably reinforces the idea that all high-energy logarithms in QCD originate from infrared enhancements: this idea underlies many of the existing approaches to the high-energy limit, and it is likely that it will bring further insights in the future.

It is easy to see that the detailed analysis provided here can be extended to scattering processes with the production of more than two partons in the final state, as well as to quark-gluon scattering with a quark Regge trajectory exchanged in the $u$ channel, as outlined in refs. $[6,7]$. This will hopefully shed further light on the interplay of highenergy and infrared factorizations, and will possibly yield high-order results relevant for high-energy precision phenomenology at colliders.

\section{Acknowledgments}

We thank S. Caron-Huot for useful discussions. This work was supported by MIUR (Italy), under contracts 2006020509_004 and 2010YJ2NYW_006; by the Research Executive Agency (REA) of the European Union, through the Initial Training Network LHCPhenoNet under contract PITN-GA-2010-264564; by the ERC grant 291377 "LHCtheory: theoretical predictions and analyses of LHC physics: advancing the precision frontier", and by the University of Torino and the Compagnia di San Paolo under contract ORTO11TPXK. LM thanks CERN, NIKHEF, and the Higgs Center for Theoretical Physics at the University 
of Edinburgh for hospitality and support during the completion of this work. LV would like to thank the Mainz Institute for Theoretical Physics (MITP) for its hospitality and support during the completion of this work.

\section{A A color basis for four-parton amplitudes in QCD}

In this section we provide orthonormal colour bases for each process we considered in the text. Most of the considerations we discussed in the paper are independent of the choice of basis in colour space, but it is useful to pick explicitly a set of tensors when dealing with the actual computation of the amplitudes in the high energy limit. In this kinematic regime, scattering amplitudes are organised conveniently by means of orthonormal bases diagonalising the operator $\mathbf{T}_{t}^{2}$. To construct them, we followed the approach of $[22,23]$, and we used the package ColorMath of ref. [24] to deal with the colour algebra.

\section{A.1 Quark-quark scattering}

The quark-quark scattering amplitude has only two color components. For $N_{c}=3$, they correspond to the exchange of a singlet or an octet in the $t$ channel, so we label the corresponding color tensors as $c_{q q}^{(1)}$ and $c_{q q}^{(8)}$; the expressions we use are however valid for generic $N_{c}$. We choose

$$
\begin{aligned}
c_{q q}^{(1)} & =\frac{1}{N_{c}} \delta_{\alpha_{1}}^{\alpha_{4}} \delta^{\alpha_{3}}{ }_{\alpha_{2}}, \\
c_{q q}^{(8)} & =\frac{2}{\sqrt{N_{c}^{2}-1}}\left(\mathbf{T}^{a}\right)^{\alpha_{4}}{ }_{\alpha_{1}}\left(\mathbf{T}_{a}\right)^{\alpha_{3}}{ }_{\alpha_{2}},
\end{aligned}
$$

where $\alpha_{i}=1, \ldots, N_{c}$ are indices in the fundamental representation of $\mathrm{SU}\left(N_{c}\right)$, while $a=$ $1, \ldots, N_{c}^{2}-1$ is in the adjoint representation, and we omit color indices on the left-hand side for simplicity. Both tensors are normalized to unity with the convention $\operatorname{Tr}\left(\mathbf{T}^{a} \mathbf{T}^{b}\right)=\frac{1}{2} \delta^{a b}$. For completeness, we report also the explicit expressions in this basis of the operators $\mathbf{T}_{t}^{2}$ and $\mathbf{T}_{s}^{2}$. The matrix $\mathbf{T}_{t}^{2}$ is diagonal by construction, while $\mathbf{T}_{s}^{2}$ mixes the different components of the amplitude in colour space. We find

$$
\mathbf{T}_{t, q q}^{2}=\left[\begin{array}{cc}
0 & 0 \\
0 & N_{c}
\end{array}\right], \quad \mathbf{T}_{s, q q}^{2}=\frac{\sqrt{N_{c}^{2}-1}}{N_{c}}\left[\begin{array}{cc}
\sqrt{N_{c}^{2}-1} & 1 \\
1 & \frac{N_{c}^{2}-3}{\sqrt{N_{c}^{2}-1}}
\end{array}\right] .
$$

\section{A.2 Gluon-gluon scattering}

The colour structure for gluon-gluon scattering is more intricate. In this case, the basis is composed of six colour tensors, which again we label with their SU(3) quantum numbers, while the expressions we give are for generic $N_{c}$. We choose

$$
\begin{aligned}
c_{g g}^{(1)} & =\frac{1}{N_{c}^{2}-1} \delta_{a_{1}}^{a_{4}} \delta_{a_{2}}^{a_{3}}, \\
c_{g g}^{\left(8_{s}\right)} & =\frac{N_{c}}{N_{c}^{2}-4} \frac{1}{\sqrt{N_{c}^{2}-1}} d^{a_{1} a_{4} b} d^{a_{2} a_{3}}{ }_{b},
\end{aligned}
$$




$$
\begin{aligned}
& c_{g g}^{(8 a)}=\frac{1}{N_{c}} \frac{1}{\sqrt{N_{c}^{2}-1}} f^{a_{1} a_{4} b} f_{b}^{a_{2} a_{3}}
\end{aligned}
$$

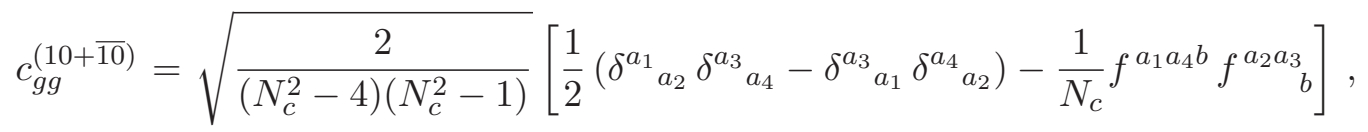

$$
\begin{aligned}
& c_{g g}^{(27)}=\frac{2}{N_{c} \sqrt{\left(N_{c}+3\right)\left(N_{c}-1\right)}}\left[-\frac{N_{c}+2}{2 N_{c}\left(N_{c}+1\right)} \delta^{a_{4}}{ }_{a_{1}} \delta^{a_{3}}{ }_{a_{2}}\right. \\
& +\frac{N_{c}+2}{4 N_{c}}\left(\delta^{a_{1}}{ }_{a_{2}} \delta^{a_{3}}{ }_{a_{4}}+\delta^{a_{3}}{ }_{a_{1}} \delta^{a_{4}}{ }_{a_{2}}\right)-\frac{N_{c}+4}{4\left(N_{c}+2\right)} d^{a_{1} a_{4} b} d^{a_{2} a_{3}}{ }_{b} \\
& \left.+\frac{1}{4}\left(d^{a_{1} a_{2} b} d_{b}^{a_{3} a_{4}}+d^{a_{1} a_{3} b} d_{b}^{a_{2} a_{4}}\right)\right] \text {, } \\
& c_{g g}^{(0)}=\frac{2}{N_{c} \sqrt{\left(N_{c}-3\right)\left(N_{c}+1\right)}}\left[\frac{N_{c}-2}{2 N_{c}\left(N_{c}-1\right)} \delta^{a_{4}}{ }_{a_{1}} \delta^{a_{3}}{ }_{a_{2}}\right. \\
& +\frac{N_{c}-2}{4 N_{c}}\left(\delta^{a_{1}}{ }_{a_{2}} \delta^{a_{3}}{ }_{a_{4}}+\delta^{a_{3}}{ }_{a_{1}} \delta^{a_{4}}{ }_{a_{2}}\right)+\frac{N_{c}-4}{4\left(N_{c}-2\right)} d^{a_{1} a_{4} b} d^{a_{2} a_{3}}{ }_{b} \\
& \left.-\frac{1}{4}\left(d^{a_{1} a_{2} b} d_{b}^{a_{3} a_{4}}+d^{a_{1} a_{3} b} d^{a_{2} a_{4}}\right)\right] .
\end{aligned}
$$

We note that it is not necessary to treat separately the two decuplet representations since they always contribute to the amplitude with the same coefficients. The tensors $c^{\left(8_{a}\right)}$ and $c^{(10+\overline{10})}$ are odd under the exchanges $a_{1} \leftrightarrow a_{4}$ and $a_{2} \leftrightarrow a_{3}$, while $c_{g g}^{(1)}, c_{g g}^{\left(8_{s}\right)}, c_{g g}^{(27)}$ and $c_{g g}^{(0)}$ are even. The last representation, as suggested by its label, does not contribute for $N_{c}=3$, since its dimensionality is given by

$$
\operatorname{dim}[\mathbf{0}]=\frac{N_{c}^{2}\left(N_{c}-3\right)\left(N_{c}+1\right)}{4},
$$

and it vanishes for $\mathrm{SU}(3)$. In the orthormal basis defined by eq. (A.3), the diagonal matrix $\mathbf{T}_{t}^{2}$ evaluates to

$$
\mathbf{T}_{t, g g}^{2}=\operatorname{diag}\left(0, N_{c}, N_{c}, 2 N_{c}, 2\left(N_{c}+1\right), 2\left(N_{c}-1\right)\right),
$$

while $\mathbf{T}_{s, g g}$ is symmetric and reads

$$
\mathbf{T}_{s, g g}^{2}=\left[\begin{array}{cccccc}
2 N_{c} & 0 & \mathcal{T}_{1,8_{a}} & 0 & 0 & 0 \\
0 & 3 N_{c} / 2 & \mathcal{T}_{8_{s}, 8_{a}} & \mathcal{T}_{8_{s}, 10} & 0 & 0 \\
\mathcal{T}_{1,8_{a}} & \mathcal{T}_{8_{s}, 8_{a}} & 3 N_{c} / 2 & 0 & \mathcal{T}_{8_{s}, 27} & \mathcal{T}_{8_{s}, 0} \\
0 & \mathcal{T}_{8_{s}, 10} & 0 & N_{c} & \mathcal{T}_{10,27} & \mathcal{T}_{10,0} \\
0 & 0 & \mathcal{T}_{8_{s}, 27} & \mathcal{T}_{10,27} & N_{c}-1 & 0 \\
0 & 0 & \mathcal{T}_{8_{s}, 0} & \mathcal{T}_{10,0} & 0 & N_{c}+1
\end{array}\right],
$$

where

$$
\mathcal{T}_{1,8_{a}}=-\frac{2 N_{c}}{\sqrt{N_{c}^{2}-1}}, \quad \mathcal{T}_{8_{s}, 8_{a}}=-\frac{N_{c}}{2}, \quad \mathcal{T}_{8_{s}, 10}=-N_{c} \sqrt{\frac{2}{N_{c}^{2}-4}},
$$




$$
\begin{aligned}
& \mathcal{T}_{8_{s}, 27}=-\sqrt{\frac{N_{c}+3}{N_{c}+1}}, \quad \mathcal{T}_{8_{s}, 0}=-\sqrt{\frac{N_{c}-3}{N_{c}-1}}, \\
& \mathcal{T}_{8_{s}, 27}=-\sqrt{\frac{N_{c}+3}{N_{c}+1}}, \\
& \mathcal{T}_{10,27}=-\sqrt{\frac{\left(N_{c}+3\right)\left(N_{c}+1\right)\left(N_{c}-2\right)}{2\left(N_{c}+2\right)}} \\
& \mathcal{T}_{10,0}=-\sqrt{\frac{\left(N_{c}-3\right)\left(N_{c}-1\right)\left(N_{c}+2\right)}{2\left(N_{c}-2\right)}} .
\end{aligned}
$$

\section{A.3 Quark-gluon scattering}

We conclude by discussing quark-gluon scattering. In this case the $t$-channel exchange takes place between a quark line and a gluon line, so in order to enumerate the relevant representations one must consider the intersection of the tensor product $\mathbf{3} \otimes \overline{\mathbf{3}}=\mathbf{1} \oplus \mathbf{8}$ with $\mathbf{8} \otimes \overline{\mathbf{8}}=\mathbf{1} \oplus \mathbf{8} \oplus \mathbf{8} \oplus \mathbf{1 0} \oplus \overline{\mathbf{1 0}} \oplus \mathbf{2 7} \oplus \mathbf{0}$, including copies of equivalent representations. This leaves the singlet and the two copies of the adjoint representation. An orthonormal basis of color tensors in this space is given by

$$
\begin{aligned}
c_{q g}^{(1)} & =\frac{1}{\sqrt{N_{c}\left(N_{c}^{2}-1\right)} \delta^{\alpha_{4}}{ }_{\alpha_{1}} \delta^{a_{3}}{ }_{a_{2}},} \\
c_{q g}^{(8 s)} & =\sqrt{\frac{2 N_{c}}{\left(N_{c}^{2}-4\right)\left(N_{c}^{2}-1\right)}}\left(T^{b}\right)^{\alpha_{4}}{ }_{\alpha_{1}} d_{b}{ }^{a_{3} a_{2}}, \\
c_{q g}^{(8 a)} & =\mathrm{i} \sqrt{\frac{2}{N_{c}\left(N_{c}^{2}-1\right)}\left(T^{b}\right)^{\alpha_{4}}{ }_{\alpha_{1}} f_{b}{ }^{a_{3} a_{2}} .}
\end{aligned}
$$

The operators $\mathbf{T}_{t}^{2}$ and $\mathbf{T}_{s}^{2}$ in this basis take the form

$$
\mathbf{T}_{t, q g}^{2}=\left[\begin{array}{ccc}
0 & 0 & 0 \\
0 & N_{c} & 0 \\
0 & 0 & N_{c}
\end{array}\right], \quad \mathbf{T}_{s, q g}^{2}=\left[\begin{array}{ccc}
\frac{3 N_{c}^{2}-1}{2 N_{c}} & 0 & -\sqrt{2} \\
0 & \frac{2 N_{c}^{2}-1}{2 N_{c}} & -\frac{\sqrt{N_{c}^{2}-4}}{2} \\
-\sqrt{2} & -\frac{\sqrt{N_{c}^{2}-4}}{2} & \frac{2 N_{c}^{2}-1}{2 N_{c}}
\end{array}\right] .
$$

\section{B Anomalous dimensions}

The discussion of Regge factorization in section 4 led us to the prediction of the divergent part of the Regge trajectory and of the impact factors, in terms of the operators appearing in the infrared factorization formula eq. (2.5). In the text we focused mostly on the formal relations connecting the two factorizations, as for example in eq. (4.10) and eq. (4.14), however one is ultimately interested in explicit results. For completeness, we give here the values of all the relevant anomalous dimensions, up to three loops.

In order to construct the infrared operators relevant to the Regge limit, defined in eq. (2.6) and eq. (2.9), we need the functions $K\left(\alpha_{s}\right), D\left(\alpha_{s}\right)$ and $B_{i}\left(\alpha_{s}\right)$, defined in eq. (2.7) 
and eq. (2.10) respectively. Performing the relevant integrals, we find

$$
\begin{aligned}
K\left(\alpha_{s}\right)= & \frac{\alpha_{s}}{\pi} \frac{\widehat{\gamma}_{K}^{(1)}}{4 \epsilon}+\left(\frac{\alpha_{s}}{\pi}\right)^{2}\left(\frac{\widehat{\gamma}_{K}^{(2)}}{8 \epsilon}-\frac{b_{0} \widehat{\gamma}_{K}^{(1)}}{32 \epsilon^{2}}\right) \\
& +\left(\frac{\alpha_{s}}{\pi}\right)^{3}\left(\frac{\widehat{\gamma}_{K}^{(3)}}{12 \epsilon}-\frac{b_{0} \widehat{\gamma}_{K}^{(2)}+b_{1} \widehat{\gamma}_{K}^{(1)}}{48 \epsilon^{2}}+\frac{b_{0}^{2} \widehat{\gamma}_{K}^{(1)}}{192 \epsilon^{3}}\right)+\mathcal{O}\left(\alpha_{s}^{4}\right), \\
D\left(\alpha_{s}\right)= & -\frac{\alpha_{s}}{\pi} \frac{\widehat{\gamma}_{K}^{(1)}}{4 \varepsilon^{2}}+\left(\frac{\alpha_{s}}{\pi}\right)^{2}\left[\frac{3 b_{0} \widehat{\gamma}_{K}^{(1)}}{64 \epsilon^{3}}-\frac{\widehat{\gamma}_{K}^{(2)}}{16 \epsilon^{2}}\right] \\
& +\left(\frac{\alpha_{s}}{\pi}\right)^{3}\left[-\frac{11 b_{0}^{2} \widehat{\gamma}_{K}^{(1)}}{1152 \epsilon^{4}}+\frac{1}{\epsilon^{3}}\left(\frac{b_{1} \widehat{\gamma}_{K}^{(1)}}{36}+\frac{5 b_{0} \widehat{\gamma}_{K}^{(2)}}{288}\right)-\frac{\widehat{\gamma}_{K}^{(3)}}{36 \epsilon^{2}}\right]+\mathcal{O}\left(\alpha_{s}^{4}\right),
\end{aligned}
$$

where $b_{i}$ are the coefficients of the perturbative expansion of the beta function which, in our normalizations, read

$$
b_{0}=\frac{11 C_{A}-4 T_{R} n_{f}}{3}, \quad b_{1}=\frac{17 C_{A}^{2}-\left(10 C_{A}+6 C_{F}\right) T_{R} n_{f}}{6},
$$

while $\widehat{\gamma}_{K}^{(i)}$ are the perturbative coefficients of the light-like cusp anomalous dimension, divided by the quadratic Casimir eigenvalue of the relevant representation. This is a universal (representation-independent) function at least up to three loops [67], given by

$$
\begin{aligned}
\widehat{\gamma}_{K}\left(\alpha_{s}\right)=2 \frac{\alpha_{s}}{\pi}+\left(\frac{\alpha_{s}}{\pi}\right)^{2}\left[\left(\frac{67}{18}-\zeta(2)\right) C_{A}-\frac{10}{9} T_{R} n_{f}\right]+ \\
+\left(\frac{\alpha_{s}}{\pi}\right)^{3}\left[\frac{C_{A}^{2}}{96}\left(490-\frac{1072}{3} \zeta(2)+88 \zeta(3)+264 \zeta(4)\right)\right. \\
+\frac{C_{A} T_{R} n_{f}}{96}\left(-\frac{1672}{9}+\frac{320}{3} \zeta(2)-224 \zeta(3)\right) \\
\left.+\frac{C_{F} T_{R} n_{f}}{32}\left(-\frac{220}{3}+64 \zeta(3)\right)-\frac{2 T_{R}^{2} n_{f}^{2}}{27}\right]+\mathcal{O}\left(\alpha_{s}^{4}\right) .
\end{aligned}
$$

Finally we note that, up to a factor of $2, B_{i}\left(\alpha_{s}\right)$ is defined by the same integral, and therefore by the same perturbative expansion, given in eq. (B.1), as $K\left(\alpha_{s}\right)$, but with the cusp anomalous dimension replaced by the collinear anomalous dimension of the relevant field, $\gamma_{i}$ with $i=q, g$. The perturbative coefficients of collinear anomalous dimensions were extracted from form factors data in [34] and they are

$$
\begin{aligned}
\gamma_{q}\left(\alpha_{s}\right)= & -\frac{3}{4} C_{F} \frac{\alpha_{s}}{\pi}+\left(\frac{\alpha_{s}}{\pi}\right)^{2}\left[\frac{C_{F}^{2}}{16}\left(-\frac{3}{2}+12 \zeta(2)-24 \zeta(3)\right)\right. \\
& \left.+\frac{C_{A} C_{F}}{16}\left(-\frac{961}{54}-11 \zeta(2)+26 \zeta(3)\right)+\frac{C_{F} T_{R} n_{f}}{16}\left(\frac{130}{27}+4 \zeta(2)\right)\right] \\
& +\left(\frac{\alpha_{s}}{\pi}\right)^{3} \frac{1}{64}\left[C_{F}^{3}\left(-\frac{29}{2}-18 \zeta(2)-68 \zeta(3)-144 \zeta(4)+32 \zeta(2) \zeta(3)+240 \zeta(5)\right)\right. \\
& +C_{F}^{2} C_{A}\left(-\frac{151}{4}+\frac{410}{3} \zeta(2)-\frac{844}{3} \zeta(3)+\frac{494}{3} \zeta(4)-16 \zeta(2) \zeta(3)-120 \zeta(5)\right)
\end{aligned}
$$




$$
\begin{aligned}
& +C_{F} C_{A}^{2}\left(-\frac{139345}{2916}-\frac{7163}{81} \zeta(2)+\frac{3526}{9} \zeta(3)-83 \zeta(4)-\frac{88}{3} \zeta(2) \zeta(3)-136 \zeta(5)\right) \\
& +C_{F}^{2} T_{R} n_{f}\left(\frac{2953}{27}-\frac{52}{3} \zeta(2)+\frac{512}{9} \zeta(3)-\frac{280}{3} \zeta(4)\right) \\
& +C_{A} C_{F} T_{R} n_{f}\left(-\frac{17318}{729}+\frac{5188}{81} \zeta(2)-\frac{1928}{27} \zeta(3)+44 \zeta(4)\right) \\
& \left.+C_{F} T_{R}^{2} n_{f}^{2}\left(\frac{9668}{729}-\frac{80}{9} \zeta(2)-\frac{32}{27} \zeta(3)\right)\right]+\mathcal{O}\left(\alpha_{s}^{4}\right), \\
\gamma_{g}\left(\alpha_{s}\right)= & -\frac{b_{0}}{4} \frac{\alpha_{s}}{\pi}+\left(\frac{\alpha_{s}}{\pi}\right)^{2}\left[\frac{C_{A}^{2}}{16}\left(-\frac{692}{27}+\frac{11}{3} \zeta(2)+2 \zeta(3)\right)\right. \\
& \left.+\frac{C_{A} T_{R} n_{f}}{16}\left(\frac{256}{27}-\frac{4}{3} \zeta(2)\right)+\frac{C_{F} T_{R} n_{f}}{4}\right] \\
& +\left(\frac{\alpha_{s}}{\pi}\right)^{3} \frac{1}{64}\left[C _ { A } ^ { 3 } \left(-\frac{97186}{729}+\frac{6109}{81} \zeta(2)+\frac{122}{3} \zeta(3)-\frac{319}{3} \zeta(4)\right.\right. \\
& +C_{A}^{2} T_{R} n_{f}\left(\frac{30715}{729}-\frac{2396}{81} \zeta(2)+\frac{712}{27} \zeta(3)+\frac{164}{3} \zeta(4)\right) \\
& +C_{A} C_{F} T_{R} n_{f}\left(\frac{2434}{27}-4 \zeta(2)-\frac{304}{9} \zeta(3)-16 \zeta(4)\right)-2 C_{F}^{2} T_{R} n_{f} \\
& \left.+C_{A} T_{R}^{2} n_{f}^{2}\left(-\frac{538}{729}+\frac{80}{27} \zeta(2)-\frac{224}{27} \zeta(3)\right)-\frac{44}{9} C_{F} T_{R}^{2} n_{f}^{2}\right]+\mathcal{O}\left(\alpha_{s}^{4}\right),
\end{aligned}
$$

which completes the list of required anomalous dimensions up to three loops.

\section{Hard functions for four-parton amplitudes in the high-energy limit}

We have now given explicitly almost all the ingredients needed to construct the high-energy limit of four-parton QCD amplitudes up to two loops, and up to three loops for infrared singular contributions. Using existing calculations, this construction can be achieved at the one-loop level up to $\mathcal{O}\left(\varepsilon^{2}\right)$, using eq. (4.6), at the two-loop level up to $\mathcal{O}\left(\varepsilon^{0}\right)$, using eq. (4.18), and at the three-loop level up to $\mathcal{O}\left(\varepsilon^{-2}\right)$, and in some cases up to $\mathcal{O}\left(\varepsilon^{-1}\right)$ using eqs. (4.46)(4.49). Specifically, all ingredients arising from infrared factorization have been given explicitly to the necessary accuracy in appendices A and B. The only missing contributions are those arising from the hard functions $H^{(n)}$, with $n=0,1,2$, which can only be extracted from explicit finite-order calculations. The necessary helicity amplitudes for the processes $q q \rightarrow q q, g g \rightarrow g g$ and $q g \rightarrow q g$ with massless quarks have been calculated up to two loops in recent years by different groups [17-21]. In this appendix we consider the high-energy limit of these amplitudes and we provide explicit expressions for the hard functions $H^{(n), l,[c]}$, for $n=0,1,2$, for all logarithmic orders and color components, and for each process. ${ }^{4}$ Inserting

\footnotetext{
${ }^{4}$ After the completion of this work, the hard functions corresponding to two-loop four-parton QCD amplitudes were extracted, without taking the high-energy limit, in ref. [62].
} 
these results in eq. (4.6) at one loop, and in eq. (4.18) at two loops, one recovers the highenergy limit of the results discussed in [17-21], while inserting them in eqs. (4.46)-(4.49) one gets a complete prediction for the poles of three-loop four-parton amplitudes in the highenergy limit, valid to leading power in $t / s$ and for poles down to $\varepsilon^{-2}$, with further predictions at single-pole level, as discussed in section 5 . Note that, as described below, to leading power in $t / s$ essentially only one helicity amplitude survives for each partonic process.

\section{C.1 Quark-quark scattering}

Quark-quark scattering is the simplest process we consider, as it contains only two colour structures, the singlet and the octet. We write the hard coefficients of the amplitude $\mathcal{M}\left(q^{+} Q^{+} \rightarrow q^{+} Q^{+}\right)$, which has leading power in the high-energy limit, by using the basis of eq. (A.1). At tree level only the octet contributes to the amplitude, and we find

$$
\begin{aligned}
& H_{q q}^{(0),[1]}=0, \\
& H_{q q}^{(0),[8]}=\frac{\sqrt{N_{c}^{2}-1}}{x},
\end{aligned}
$$

where here and below $x=\frac{t}{s}$. Next we consider the one-loop amplitude, expanded up to $O\left(\epsilon^{2}\right)$. Leading logarithmic terms at this perturbative order are given only by the Regge trajectory: indeed, we find vanishing LL colour-singlet and octet components in $d=4$, as explained in the text. More precisely,

$$
\begin{aligned}
& H_{q q}^{(1), 1,[1]}=0 \\
& H_{q q}^{(1), 1,[8]}=-\frac{N_{c} \sqrt{N_{c}^{2}-1}}{24 x} \epsilon(6 \zeta(2)+28 \epsilon \zeta(3)) .
\end{aligned}
$$

Turning to non-logarithmic terms at one loop, we find ${ }^{5}$

$$
\begin{aligned}
H_{q q}^{(1), 0,[1]}= & -\mathrm{i} \pi \frac{N_{c}^{2}-1}{24 N_{c} x} \epsilon(6 \zeta(2)+28 \epsilon \zeta(3)) \\
H_{q q}^{(1), 0,[8]}= & \frac{\sqrt{N_{c}^{2}-1}}{x}\left\{\left(\frac{13}{36}+\frac{7}{4} \zeta(2)\right) N_{c}+\left(2-\frac{1}{4} \zeta(2)\right) \frac{1}{N_{c}}-\frac{5}{18} n_{f}\right. \\
+ & \epsilon\left[\left(\frac{20}{27}-\frac{1}{12} \zeta(2)+\frac{5}{3} \zeta(3)\right) N_{c}+\left(4-\frac{3}{8} \zeta(2)-\frac{7}{6} \zeta(3)\right) \frac{1}{N_{c}}\right. \\
& \left.+\left(-\frac{14}{27}+\frac{1}{12} \zeta(2)\right) n_{f}\right] \\
+ & \epsilon^{2}\left[\left(\frac{121}{81}-\frac{13}{72} \zeta(2)-\frac{7}{18} \zeta(3)+\frac{35}{32} \zeta(4)\right) N_{c}\right. \\
& \left.+\left(8-\zeta(2)-\frac{7}{4} \zeta(3)-\frac{47}{32} \zeta(4)\right) \frac{1}{N_{c}}+\left(-\frac{82}{81}+\frac{5}{36} \zeta(2)+\frac{7}{18} \zeta(3)\right) n_{f}\right] \\
+ & \left.\mathrm{i} \pi \frac{1}{12 N_{c}} \epsilon(6 \zeta(2)+28 \epsilon \zeta(3))\right\} .
\end{aligned}
$$

\footnotetext{
${ }^{5}$ Throughout appendix $\mathrm{C}$ we have explicitly set $T_{R}=1 / 2$.
} 
Now we consider the two-loop quark-quark scattering amplitude, where only terms up to $\mathcal{O}\left(\varepsilon^{0}\right)$ are available. The leading-logarithmic hard functions vanish again in the high-energy limit, as discussed in the text. Indeed we find

$$
\begin{aligned}
& H_{q q}^{(2), 2,[1]}=0, \\
& H_{q q}^{(2), 2,[8]}=0 .
\end{aligned}
$$

At two loops, next-to-leading logarithms in the octet component are related to the gluon Regge trajectory, while the singlet component vanishes, so that

$$
\begin{aligned}
& H_{q q}^{(2), 1,[1]}=0 \\
& H_{q q}^{(2), 1,[8]}=-N_{c} \frac{\sqrt{N_{c}^{2}-1}}{x} \frac{(27 \zeta(3)-202) N_{c}+28 n_{f}}{216} .
\end{aligned}
$$

Finally, the non-logarithmic hard functions $H^{(2), 0,[c]}$ are given by

$$
\begin{aligned}
H_{q q}^{(2), 0,[1]}= & \mathrm{i} \pi \frac{N_{c}^{2}-1}{N_{c} x} \frac{(202+324 \zeta(2)+135 \zeta(3)) N_{c}-28 n_{f}}{216}, \\
H_{q q}^{(2), 0,[8]}= & \frac{\sqrt{N_{c}^{2}-1}}{x}\left\{\left(\frac{23213}{20736}+\frac{437}{144} \zeta(2)+\frac{41}{72} \zeta(3)+\frac{105}{64} \zeta(4)\right) N_{c}^{2}\right. \\
& +\frac{30659}{5184}+\frac{833}{288} \zeta(2)-\frac{205}{144} \zeta(3)-\frac{41}{32} \zeta(4) \\
& +\left(\frac{511}{256}+\frac{13}{32} \zeta(2)-\frac{15}{16} \zeta(3)-\frac{39}{64} \zeta(4)\right) \frac{1}{N_{c}^{2}} \\
& -\left(\frac{455}{432}+\frac{107}{144} \zeta(2)+\frac{23}{72} \zeta(3)\right) N_{c} n_{f} \\
& -\left(\frac{685}{648}+\frac{13}{144} \zeta(2)+\frac{19}{72} \zeta(3)\right) \frac{n_{f}}{N_{c}}+\frac{25}{324} n_{f}^{2} \\
& \left.+\mathrm{i} \pi\left(-\frac{101}{54}+\frac{1}{4} \zeta(3)+\frac{7}{27} \frac{n_{f}}{N_{c}}\right)\right\} .
\end{aligned}
$$

\section{C.2 Gluon-gluon scattering}

The gluon-gluon scattering amplitude has more structures: by using the colour basis described in appendix A, we identify two odd components (the antisymmetric octet, and the direct sum of the decuplet and its complex conjugate), and four even components (the singlet, the symmetric octet, and the representations we label with $\mathbf{2 7}$ and $\mathbf{0}$ ). Here we consider the scattering processes $\mathcal{M}\left(g^{+} g^{-} \rightarrow g^{+} g^{-}\right)=\mathcal{M}\left(g^{+} g^{+} \rightarrow g^{-} g^{-}\right)$, which are leading in the high-energy limit. We begin with the tree-level amplitude: at this order only the antisymmetric octet contributes to the high energy limit, and we find

$$
\begin{aligned}
& H_{g g}^{(0),[1]}=H_{g g}^{(0),\left[8_{s}\right]}=H_{g g}^{(0),[10+\overline{10}]}=H_{g g}^{(0),[27]}=H_{g g}^{(0),[0]}=0, \\
& H_{g g}^{(0),[8]}=-2 \frac{N_{c} \sqrt{N_{c}^{2}-1}}{x} .
\end{aligned}
$$

Leading-logarithmic one-loop hard parts, as expected, also vanish in $d=4$. More precisely

$$
H_{g g}^{(1), 1,[1]}=H_{g g}^{(1), 1,\left[8_{s}\right]}=H_{g g}^{(1), 1,[10+\overline{10}]}=H_{g g}^{(1), 1,[27]}=H_{g g}^{(1), 1,[0]}=0,
$$




$$
H_{g g}^{(1), 1,\left[8_{a}\right]}=\frac{N_{c}^{2} \sqrt{N_{c}^{2}-1}}{12 x} \epsilon(6 \zeta(2)+28 \epsilon \zeta(3)),
$$

The one-loop amplitude is completed by the vector $H^{(1), 0}$, whose components, expanded up to $O\left(\epsilon^{2}\right)$, are given by

$$
\begin{aligned}
& H_{g g}^{(1), 0,[1]}=-\mathrm{i} \pi \frac{N_{c}^{2}}{6 x} \epsilon(6 \zeta(2)+28 \epsilon \zeta(3)), \\
& H_{g g}^{(1), 0,\left[8_{s}\right]}=-\mathrm{i} \pi \frac{N_{c}^{2} \sqrt{N_{c}^{2}-1}}{24 x} \epsilon(6 \zeta(2)+28 \epsilon \zeta(3)), \\
& H_{g g}^{(1), 0,\left[8_{a}\right]}=\frac{N_{c} \sqrt{N_{c}^{2}-1}}{x}\left\{\left(\frac{67}{18}-4 \zeta(2)\right) N_{c}-\frac{5}{9} n_{f}+\epsilon\left[\left(\frac{202}{27}-\frac{17}{3} \zeta(3)\right) N_{c}\right.\right. \\
& \left.-\frac{28}{27} n_{f}-\frac{b_{0}}{4} \zeta(2)\right]+\epsilon^{2}\left[\left(\frac{1214}{81}-\frac{67}{36} \zeta(2)-\frac{77}{18} \zeta(3)-\frac{41}{8} \zeta(4)\right) N_{c}\right. \\
& \left.\left.+\left(-\frac{164}{81}+\frac{5}{18} \zeta(2)+\frac{7}{9} \zeta(3)\right) n_{f}\right]-\mathrm{i} \pi \frac{N_{c}}{24} \epsilon(6 \zeta(2)+28 \epsilon \zeta(3))\right\}, \\
& H_{g g}^{(1), 0,[10+\overline{10}]}=0, \\
& H_{g g}^{(1), 0,[27]}=-\mathrm{i} \pi \frac{N_{c} \sqrt{\left(N_{c}+3\right)\left(N_{c}-1\right)}}{12 x} \epsilon(6 \zeta(2)+28 \epsilon \zeta(3)), \\
& H_{g g}^{(1), 0,[0]}=-\mathrm{i} \pi \frac{N_{c} \sqrt{\left(N_{c}-3\right)\left(N_{c}+1\right)}}{12 x} \epsilon(6 \zeta(2)+28 \epsilon \zeta(3)) .
\end{aligned}
$$

At two loops, leading-logarithmic hard functions vanish to $\mathcal{O}\left(\varepsilon^{0}\right)$,

$$
H_{g g}^{(2), 2,[k]}=0,
$$

while at NLL accuracy we find

$$
\begin{aligned}
H_{g g}^{(2), 1,[1]} & =H_{g g}^{(2), 1,\left[8_{s}\right]}=H_{g g}^{(2), 1,[10+\overline{10}]}=H_{g g}^{(1), 2,[27]}=H_{g g}^{(2), 2,[0]}=0, \\
H_{g g}^{(2), 1,\left[8_{a}\right]} & =-\frac{N_{c}^{2} \sqrt{N_{c}^{2}-1}}{x}\left[\left(\frac{101}{54}-\frac{1}{4} \zeta(3)\right) N_{c}-\frac{7}{27} n_{f}\right] .
\end{aligned}
$$

Finally, the components of non-logarithmic hard function $H^{(2), 0}$ are

$$
\begin{aligned}
& H_{g g}^{(2), 0,[1]}=\mathrm{i} \pi \frac{1}{x}\left\{\left[\left(\frac{265}{54}+\frac{5}{2} \zeta(3)\right) N_{c}^{3}-\frac{139}{216} N_{c}^{2} n_{f}+\frac{7}{6} n_{f}+\frac{1}{8} \frac{n_{f}}{N_{c}^{2}}\right]+2 \zeta(2) \frac{11 N_{c}^{3}+2 n_{f}}{3}\right\}, \\
& H_{g g}^{(2), 0,\left[8_{s}\right]}=\mathrm{i} \pi \frac{\sqrt{N_{c}^{2}-1}}{x}\left[\left(\frac{101}{108}-\frac{1}{8} \zeta(3)\right) N_{c}^{3}-\frac{7}{54} N_{c}^{2} n_{f}\right. \\
& \left.+\left(\frac{29}{12}+\frac{4}{3} \zeta(2)\right) n_{f}+\frac{1}{4} \frac{n_{f}}{N_{c}^{2}}\right] \\
& H_{g g}^{(2), 0,\left[8_{a}\right]}=\frac{\sqrt{N_{c}^{2}-1}}{x}\left[\left(\frac{11093}{1296}-\frac{67}{72} \zeta(2)-\frac{22}{9} \zeta(3)-\frac{37}{8} \zeta(4)\right) N_{c}^{3}\right. \\
& +\left(-\frac{4849}{2592}+\frac{5}{36} \zeta(2)-\frac{1}{18} \zeta(3)\right) N_{c}^{2} n_{f}+\left(\frac{55}{96}-\frac{1}{2} \zeta(3)\right) n_{f}
\end{aligned}
$$




$$
\begin{aligned}
& \left.+\mathrm{i} \pi \frac{N_{c}^{2}}{216}\left((202-27 \zeta(3)) N_{c}-28 n_{f}\right)\right] \\
& H_{g g}^{(2), 0,[10+\overline{10}]}=0, \\
& H_{g g}^{(2), 0,[27]}=\mathrm{i} \pi \frac{\sqrt{\left(N_{c}+3\right)\left(N_{c}-1\right)} N_{c}}{x}\left[\frac{5}{8} N_{c}^{2}+\left(\frac{683}{216}-\frac{11}{3} \zeta(2)-\frac{7}{4} \zeta(3)\right) N_{c}\right. \\
& \left.-\frac{11}{16} N_{c} n_{f}+\frac{1}{12}-\frac{22}{3} \zeta(2)-3 \zeta(3)+\left(-\frac{23}{54}+\frac{2}{3} \zeta(2)\right) n_{f}-\frac{1}{16} \frac{n_{f}}{N_{c}}\right], \\
& H_{g g}^{(2), 0,[0]}=\mathrm{i} \pi \frac{\sqrt{\left(N_{c}-3\right)\left(N_{c}+1\right)} N_{c}}{x}\left[-\frac{5}{8} N_{c}^{2}+\left(\frac{683}{216}-\frac{11}{3} \zeta(2)-\frac{7}{4} \zeta(3)\right) N_{c}\right. \\
& \left.+\frac{11}{16} N_{c} n_{f}-\frac{1}{12}+\frac{22}{3} \zeta(2)+3 \zeta(3)+\left(-\frac{23}{54}+\frac{2}{3} \zeta(2)\right) n_{f}+\frac{1}{16} \frac{n_{f}}{N_{c}}\right] .
\end{aligned}
$$

\section{C.3 Quark-gluon scattering}

To conclude, we provide the hard functions up to two loops for quark-gluon scattering amplitudes. In this case there are three color components, corresponding to a singlet and two octets, since one has to take the intersection of the vector spaces defined by the tensor products $\mathbf{3} \otimes \overline{\mathbf{3}}$ and $\mathbf{8} \otimes \mathbf{8}$, including separately all equivalent representations. The helicity amplitudes which are leading in the high-energy limit are $\mathcal{M}\left(q^{+} g^{-} \rightarrow q^{+} g^{-}\right)=$ $-\mathcal{M}\left(q^{+} g^{+} \rightarrow q^{+} g^{+}\right)$, and in the following we give the hard functions for the process $\mathcal{M}\left(q^{+} g^{-} \rightarrow q^{+} g^{-}\right)$. The tree-level amplitude is given by

$$
\begin{aligned}
H_{q g}^{(0),[1]} & =H_{q g}^{(0),\left[8_{s}\right]}=0, \\
H_{q g}^{(0),\left[8_{a}\right]} & =\frac{\sqrt{2 N_{c}\left(N_{c}^{2}-1\right)}}{x} .
\end{aligned}
$$

At one loop we have the leading logarithmic functions

$$
\begin{aligned}
H_{q g}^{(1), 1,[1]} & =H_{q g}^{(1), 1,\left[8_{s}\right]}=0 \\
H_{q g}^{(1), 1,\left[8_{a}\right]} & =-\frac{N_{c} \sqrt{2 N_{c}\left(N_{c}^{2}-1\right)}}{24 x} \epsilon(6 \zeta(2)+28 \epsilon \zeta(3)),
\end{aligned}
$$

while the NLL result, up to $\mathcal{O}\left(\varepsilon^{2}\right)$, is given by

$$
\begin{aligned}
H_{q g}^{(1), 0,[1]}= & \mathrm{i} \pi \frac{\sqrt{N_{c}\left(N_{c}^{2}-1\right)}}{12 x} \epsilon(6 \zeta(2)+28 \epsilon \zeta(3)), \\
H_{q g}^{(1), 0,\left[8_{s}\right]}= & \mathrm{i} \pi \frac{\sqrt{2 N_{c}\left(N_{c}^{2}-1\right)\left(N_{c}^{2}-4\right)}}{48 x} \epsilon(6 \zeta(2)+28 \epsilon \zeta(3)) \\
H_{q g}^{(1), 0,\left[8_{a}\right]}= & \frac{\sqrt{2 N_{c}\left(N_{c}^{2}-1\right)}}{x}\left\{\left(-\frac{3}{4}+\frac{15}{8} \zeta(2)\right) N_{c}+\left(1-\frac{1}{8} \zeta(2)\right) \frac{1}{N_{c}}\right. \\
& +\epsilon\left[\left(-\frac{3}{2}+\frac{3}{16} \zeta(2)+\frac{9}{4} \zeta(3)\right) N_{c}+\left(2-\frac{3}{16} \zeta(2)-\frac{7}{12} \zeta(3)\right) \frac{1}{N_{c}}\right] \\
& +\epsilon^{2}\left[\left(-3+\frac{3}{8} \zeta(2)+\frac{7}{8} \zeta(3)+\frac{117}{64} \zeta(4)\right) N_{c}\right.
\end{aligned}
$$




$$
\begin{aligned}
&+\left.\left(4-\frac{1}{2} \zeta(2)-\frac{7}{8} \zeta(3)-\frac{47}{64} \zeta(4)\right) \frac{1}{N_{c}}\right] \\
&\left.+\mathrm{i} \pi \frac{N_{c}}{48} \epsilon(6 \zeta(2)+28 \epsilon \zeta(3))\right\} .
\end{aligned}
$$

At two loops, leading-logarithmic contributions to the hard functions vanish to $\mathcal{O}\left(\varepsilon^{0}\right)$,

$$
H_{q g}^{(2), 2,[k]}=0 .
$$

At NLL accuracy, on the other hand, the singlet and symmetric octet components vanish, but the antisymmetric octet component $H^{(2), 1,\left[8_{a}\right]}$ is related to the finite part of the twoloop Regge trajectory, and one finds

$$
\begin{aligned}
H_{q g}^{(2), 1,[1]} & =H_{q g}^{(2), 1,\left[8_{s}\right]}=0 \\
H_{q g}^{(2), 1,\left[8_{a}\right]} & =-\frac{N_{c} \sqrt{2 N_{c}\left(N_{c}^{2}-1\right)}}{216 x}\left[(-202+27 \zeta(3)) N_{c}+28 n_{f}\right] .
\end{aligned}
$$

Finally, all colour components of $H^{(2), 0}$ are non vanishing, and are given by

$$
\begin{aligned}
H_{q g}^{(2), 0,[1]}= & \mathrm{i} \pi \frac{\sqrt{N_{c}\left(N_{c}^{2}-1\right)}}{N_{c} x}\left[\left(-\frac{55}{27}-\frac{10}{3} \zeta(2)-\frac{5}{4} \zeta(3)\right) N_{c}^{2}-\frac{3}{16} \frac{1}{N_{c}^{2}}+\frac{83}{216} N_{c} n_{f}\right. \\
& \left.-\left(\frac{1}{6}+\frac{1}{3} \zeta(2)\right) \frac{n_{f}}{N_{c}}-\frac{1}{16}\right] \\
H_{q g}^{(2), 0,\left[8_{s}\right]}= & \mathrm{i} \pi \frac{\sqrt{2 N_{c}\left(N_{c}^{2}-1\right)\left(N_{c}^{2}-4\right)}}{N_{c} x}\left[\left(-\frac{101}{216}+\frac{\zeta(3)}{16}\right) N_{c}^{2}-\frac{3}{32} \frac{1}{N_{c}^{2}}+\frac{7}{108} N_{c} n_{f}\right. \\
& \left.-\left(\frac{1}{6}+\frac{1}{3} \zeta(2)\right) \frac{n_{f}}{N_{c}}-\frac{3}{32}\right], \\
H_{q g}^{(2), 0,\left[8_{a}\right]}= & \frac{\sqrt{2 N_{c}\left(N_{c}^{2}-1\right)}\left\{\left(-\frac{30377}{13824}+\frac{17}{9} \zeta(2)+\frac{43}{48} \zeta(3)+\frac{501}{256} \zeta(4)\right) N_{c}^{2}\right.}{x} \\
& +\left(\frac{255}{512}+\frac{21}{64} \zeta(2)-\frac{15}{32} \zeta(3)-\frac{83}{256} \zeta(4)\right) \frac{1}{N_{c}^{2}} \\
& +\left(\frac{863}{3456}-\frac{127}{288} \zeta(2)-\frac{7}{48} \zeta(3)\right) N_{c} n_{f} \\
& -\left(\frac{4085}{10368}+\frac{23}{288} \zeta(2)+\frac{1}{144} \zeta(3)\right) \frac{n_{f}}{N_{c}} \\
& +\frac{19139}{10368}+\frac{985}{576} \zeta(2)-\frac{205}{288} \zeta(3)-\frac{87}{128} \zeta(4) \\
& \left.+\mathrm{i} \pi \frac{N_{c}}{432}\left[(-202+27 \zeta(3)) N_{c}+28 n_{f}\right]\right\}
\end{aligned}
$$

\section{Infrared singularities for singlet exchange}

In section 5 we used the dipole formula to investigate the structure of infrared singularities for the $t$-channel exchange of colour representations other than the octet, providing examples at three and four loops. In this appendix, as a further example of the dipole formula 
at work, and for future reference, we give explicit expressions for the infrared singularities in the case of singlet exchange. We begin with the poles of one-loop amplitudes. Leading logarithms are given by (4.6),

$$
M^{(1), 1}=K^{(1)} \mathbf{O}_{t} H^{(0)}+\mathcal{O}\left(\epsilon^{0}\right),
$$

where the operators $\mathbf{O}_{t}$ and hard parts $H^{(0)}$ for the three processes are given respectively in eqs. (A.2) and (C.1), (A.5) and (C.7), (A.10) and (C.13). The coefficient $K^{(1)}=\frac{1}{2 \epsilon}$ can be extracted by replacing eq. (B.3) in eq. (B.1). For example, in quark-quark scattering one finds the simple expressions

$$
M_{q q}^{(1), 1}=\frac{1}{2 \epsilon}\left[\begin{array}{ll}
0 & 0 \\
0 & N_{c}
\end{array}\right]\left(\begin{array}{c}
0 \\
\frac{\sqrt{N_{c}^{2}-1}}{x}
\end{array}\right)=\left(\begin{array}{c}
0 \\
\frac{N_{c} \sqrt{N_{c}^{2}-1}}{2 \epsilon x}
\end{array}\right)+\mathcal{O}\left(\epsilon^{0}\right) .
$$

The first component of the vector corresponds to the exchange of a color singlet in the $t$ channel and, as expected, it vanishes at leading logarithmic accuracy. The same result holds for gluon-gluon and quark-gluon scattering. We then use the same procedure for next-to-leading logarithms, by replacing the operators $\mathbf{O}_{s-u}$ in the proper representation and the anomalous dimensions we find in appendix B in the first expression of eq. (4.6). Singlet components in this case are

$$
\begin{aligned}
& M_{q q}^{(1), 0,[1]}=\mathrm{i} \pi \frac{s}{t} \frac{N_{c}^{2}-1}{2 N_{c}} \frac{1}{\epsilon}+\mathcal{O}\left(\epsilon^{0}\right) \\
& M_{g g}^{(1), 0,[1]}=\mathrm{i} \pi \frac{s}{t} 2 N_{c}^{2} \frac{1}{\epsilon}+\mathcal{O}\left(\epsilon^{0}\right) \\
& M_{q g}^{(1), 0,[1]}=-\mathrm{i} \pi \frac{s}{t} \sqrt{N_{c}\left(N_{c}^{2}-1\right)} \frac{1}{\epsilon}+\mathcal{O}\left(\epsilon^{0}\right) .
\end{aligned}
$$

We next consider two-loop amplitudes, isolating leading and subleading logarithms. The singularities are constructed according to eq. (4.18). We find again that leading logarithms have just the (antisymmetric) octet component, while the next-to-leading terms are

$$
\begin{aligned}
& M_{q q}^{(2), 1,[1]}=\mathrm{i} \pi \frac{s}{t} \frac{N_{c}^{2}-1}{8 \epsilon^{2}}+\mathcal{O}\left(\epsilon^{0}\right) \\
& M_{g g}^{(2), 1,[1]}=\mathrm{i} \pi \frac{s}{t} \frac{N_{c}^{3}}{2 \epsilon^{2}}+\mathcal{O}\left(\epsilon^{0}\right) \\
& M_{q g}^{(2), 1,[1]}=-\mathrm{i} \pi \frac{s}{t} \frac{N_{c} \sqrt{N_{c}\left(N_{c}^{2}-1\right)}}{4 \epsilon^{2}}+\mathcal{O}\left(\epsilon^{0}\right) .
\end{aligned}
$$

Finally, at next-to-next-to-leading logarithmic accuracy we find

$$
\begin{aligned}
M_{q q}^{(2), 0,[1]}= & \frac{s}{t} \frac{N_{c}^{2}-1}{N_{c}}\left\{-\mathrm{i} \pi \frac{N_{c}^{2}-1}{4 N_{c} \epsilon^{3}}+\frac{1}{\epsilon^{2}}\left[\frac{3}{2} \frac{1}{N_{c}} \zeta(2)+\mathrm{i} \pi\left(-\frac{29}{48} N_{c}+\frac{1}{24} n_{f}+\frac{3}{8} \frac{1}{N_{c}}\right)\right]\right. \\
& \left.+\mathrm{i} \pi \frac{1}{\epsilon}\left[N_{c}\left(\frac{31}{48}+\frac{7}{8} \zeta(2)\right)-\frac{5}{24} n_{f}+\frac{1}{N_{c}}\left(1-\frac{1}{4} \zeta(2)\right)\right]\right\},
\end{aligned}
$$




$$
\begin{aligned}
M_{g g}^{(2), 0,[1]}= & \frac{s}{t} N_{c}^{2}\left\{-2 \mathrm{i} \pi \frac{N_{c}}{\epsilon^{3}}+\frac{1}{\epsilon^{2}}\left[\frac{3}{2} N_{c} \zeta(2)+\mathrm{i} \pi\left(-\frac{55}{12} N_{c}+\frac{5}{6} n_{f}\right)\right]\right. \\
& \left.+\mathrm{i} \pi \frac{1}{\epsilon}\left[N_{c}\left(-\frac{67}{36}+\frac{9}{2} \zeta(2)\right)+\frac{5}{18} n_{f}\right]\right\}, \\
M_{q g}^{(2), 0,[1]}= & \frac{s}{t} \sqrt{\frac{N_{c}^{2}-1}{N_{c}}}\left\{\mathrm{i} \pi \frac{3 N_{c}^{2}-1}{4 \epsilon^{3}}+\frac{1}{\epsilon^{2}}\left[-\frac{3}{4} N_{c}^{2} \zeta(2)+\mathrm{i} \pi\left(\frac{7}{4} N_{c}^{2}-\frac{1}{4} n_{f} N_{c}-\frac{3}{8}\right)\right]\right. \\
& \left.-\mathrm{i} \pi \frac{1}{\epsilon}\left[N_{c}^{2}\left(\frac{13}{72}+2 \zeta(2)\right)-\frac{5}{36} n_{f} N_{c}+1-\frac{1}{4} \zeta(2)\right]\right\} .
\end{aligned}
$$

Open Access. This article is distributed under the terms of the Creative Commons Attribution License (CC-BY 4.0), which permits any use, distribution and reproduction in any medium, provided the original author(s) and source are credited.

\section{References}

[1] I.I. Balitsky, L.N. Lipatov and V.S. Fadin, Regge processes in non-Abelian gauge theories (in Russian), in Leningrad 1979, Proceedings, Physics Of Elementary Particles, Leningrad Russia (1979), pg. 109 [INSPIRE].

[2] V.S. Fadin, R. Fiore, M.G. Kozlov and A.V. Reznichenko, Proof of the multi-Regge form of QCD amplitudes with gluon exchanges in the NLA, Phys. Lett. B 639 (2006) 74 [hep-ph/0602006] [INSPIRE].

[3] V.S. Fadin and L.N. Lipatov, Radiative corrections to QCD scattering amplitudes in a multi-Regge kinematics, Nucl. Phys. B 406 (1993) 259 [INSPIRE].

[4] V. Del Duca and E.W.N. Glover, The high-energy limit of QCD at two loops, JHEP 10 (2001) 035 [hep-ph/0109028] [INSPIRE].

[5] P.D.B. Collins, An introduction to Regge theory and high-energy physics, Cambridge University Press, Cambridge U.K. (1977) [InSPIRE].

[6] V. Del Duca, C. Duhr, E. Gardi, L. Magnea and C.D. White, The infrared structure of gauge theory amplitudes in the high-energy limit, JHEP 12 (2011) 021 [arXiv:1109.3581] [INSPIRE].

[7] V. Del Duca, C. Duhr, E. Gardi, L. Magnea and C.D. White, An infrared approach to Reggeization, Phys. Rev. D 85 (2012) 071104 [arXiv:1108.5947] [INSPIRE].

[8] G.P. Korchemsky, On near forward high-energy scattering in $Q C D$, Phys. Lett. B 325 (1994) 459 [hep-ph/9311294] [INSPIRE].

[9] I.A. Korchemskaya and G.P. Korchemsky, High-energy scattering in QCD and cross singularities of Wilson loops, Nucl. Phys. B 437 (1995) 127 [hep-ph/9409446] [INSPIRE].

[10] I.A. Korchemskaya and G.P. Korchemsky, Evolution equation for gluon Regge trajectory, Phys. Lett. B 387 (1996) 346 [hep-ph/9607229] [INSPIRE].

[11] V. Del Duca, G. Falcioni, L. Magnea and L. Vernazza, High-energy QCD amplitudes at two loops and beyond, Phys. Lett. B 732 (2014) 233 [arXiv:1311.0304] [INSPIRE]. 
[12] V. Del Duca, G. Falcioni, L. Magnea and L. Vernazza, Beyond Reggeization for two- and three-loop QCD amplitudes, PoS(RADCOR 2013) 046 [arXiv:1312.5098] [INSPIRE].

[13] I. Balitsky, Factorization and high-energy effective action, Phys. Rev. D 60 (1999) 014020 [hep-ph/9812311] [INSPIRE].

[14] I. Balitsky, High-energy QCD and Wilson lines, in At the frontier of particle physics, vol. 2, M. Shifman ed., World Scientific, Singapore (2001), pg. 1237 [hep-ph/0101042] [INSPIRE].

[15] T. Kucs, Scattering amplitudes in high-energy QCD, Phys. Rev. D 69 (2004) 054016 [hep-ph/0307141] [INSPIRE].

[16] S. Caron-Huot, When does the gluon reggeize?, arXiv:1309.6521 [INSPIRE].

[17] Z. Bern, A. De Freitas and L.J. Dixon, Two loop helicity amplitudes for gluon-gluon scattering in QCD and supersymmetric Yang-Mills theory, JHEP 03 (2002) 018 [hep-ph/0201161] [INSPIRE].

[18] Z. Bern, A. De Freitas and L.J. Dixon, Two loop helicity amplitudes for quark gluon scattering in QCD and gluino gluon scattering in supersymmetric Yang-Mills theory, JHEP 06 (2003) 028 [Erratum ibid. 04 (2014) 112] [hep-ph/0304168] [INSPIRE].

[19] A. De Freitas and Z. Bern, Two-loop helicity amplitudes for quark-quark scattering in QCD and gluino-gluino scattering in supersymmetric Yang-Mills theory, JHEP 09 (2004) 039 [hep-ph/0409007] [INSPIRE].

[20] E.W.N. Glover and M.E. Tejeda-Yeomans, Two loop QCD helicity amplitudes for massless quark massless gauge boson scattering, JHEP 06 (2003) 033 [hep-ph/0304169] [INSPIRE].

[21] E.W.N. Glover, Two loop QCD helicity amplitudes for massless quark quark scattering, JHEP 04 (2004) 021 [hep-ph/0401119] [INSPIRE].

[22] M. Beneke, P. Falgari and C. Schwinn, Soft radiation in heavy-particle pair production: all-order colour structure and two-loop anomalous dimension, Nucl. Phys. B 828 (2010) 69 [arXiv: 0907.1443] [INSPIRE].

[23] W. Beenakker et al., Towards NNLL resummation: hard matching coefficients for squark and gluino hadroproduction, JHEP 10 (2013) 120 [arXiv:1304.6354] [INSPIRE].

[24] M. Sjödahl, ColorMath - a package for color summed calculations in $\mathrm{SU}\left(N_{c}\right)$, Eur. Phys. J. C 73 (2013) 2310 [arXiv:1211.2099] [inSPIRE].

[25] S. Catani, The singular behavior of QCD amplitudes at two loop order, Phys. Lett. B 427 (1998) 161 [hep-ph/9802439] [INSPIRE].

[26] G.F. Sterman and M.E. Tejeda-Yeomans, Multiloop amplitudes and resummation, Phys. Lett. B 552 (2003) 48 [hep-ph/0210130] [INSPIRE].

[27] L.J. Dixon, L. Magnea and G.F. Sterman, Universal structure of subleading infrared poles in gauge theory amplitudes, JHEP 08 (2008) 022 [arXiv:0805.3515] [INSPIRE].

[28] I. Feige and M.D. Schwartz, Hard-soft-collinear factorization to all orders, Phys. Rev. D 90 (2014) 105020 [arXiv:1403.6472] [INSPIRE].

[29] L. Magnea and G.F. Sterman, Analytic continuation of the Sudakov form-factor in QCD, Phys. Rev. D 42 (1990) 4222 [INSPIRE].

[30] S.M. Aybat, L.J. Dixon and G.F. Sterman, The two-loop anomalous dimension matrix for soft gluon exchange, Phys. Rev. Lett. 97 (2006) 072001 [hep-ph/0606254] [INSPIRE]. 
[31] S.M. Aybat, L.J. Dixon and G.F. Sterman, The two-loop soft anomalous dimension matrix and resummation at next-to-next-to leading pole, Phys. Rev. D 74 (2006) 074004 [hep-ph/0607309] [INSPIRE].

[32] T. Becher and M. Neubert, Infrared singularities of scattering amplitudes in perturbative QCD, Phys. Rev. Lett. 102 (2009) 162001 [arXiv:0901.0722] [INSPIRE].

[33] E. Gardi and L. Magnea, Factorization constraints for soft anomalous dimensions in QCD scattering amplitudes, JHEP 03 (2009) 079 [arXiv:0901.1091] [INSPIRE].

[34] T. Becher and M. Neubert, On the structure of infrared singularities of gauge-theory amplitudes, JHEP 06 (2009) 081 [Erratum ibid. 11 (2013) 024] [arXiv:0903.1126] [INSPIRE].

[35] E. Gardi and L. Magnea, Infrared singularities in QCD amplitudes, Nuovo Cim. C32N5-6 (2009) 137 [arXiv:0908.3273] [INSPIRE].

[36] G.P. Korchemsky and A.V. Radyushkin, Loop space formalism and renormalization group for the infrared asymptotics of QCD, Phys. Lett. B 171 (1986) 459 [INSPIRE].

[37] G.P. Korchemsky and A.V. Radyushkin, Renormalization of the Wilson loops beyond the leading order, Nucl. Phys. B 283 (1987) 342 [INSPIRE].

[38] L.J. Dixon, E. Gardi and L. Magnea, On soft singularities at three loops and beyond, JHEP 02 (2010) 081 [arXiv:0910.3653] [INSPIRE].

[39] V. Ahrens, M. Neubert and L. Vernazza, Structure of infrared singularities of gauge-theory amplitudes at three and four loops, JHEP 09 (2012) 138 [arXiv:1208.4847] [INSPIRE].

[40] E. Gardi, From webs to polylogarithms, JHEP 04 (2014) 044 [arXiv: 1310.5268] [INSPIRE].

[41] G. Falcioni, E. Gardi, M. Harley, L. Magnea and C.D. White, Multiple gluon exchange webs, JHEP 10 (2014) 010 [arXiv: 1407.3477] [INSPIRE].

[42] A. Grozin, J.M. Henn, G.P. Korchemsky and P. Marquard, The three-loop cusp anomalous dimension in $Q C D$, arXiv: 1409.0023 [INSPIRE].

[43] Z. Bern, L.J. Dixon and V.A. Smirnov, Iteration of planar amplitudes in maximally supersymmetric Yang-Mills theory at three loops and beyond, Phys. Rev. D 72 (2005) 085001 [hep-th/0505205] [INSPIRE].

[44] L. Magnea, Analytic resummation for the quark form-factor in $Q C D$, Nucl. Phys. B 593 (2001) 269 [hep-ph/0006255] [INSPIRE].

[45] V. Del Duca and C.R. Schmidt, Virtual next-to-leading corrections to the impact factors in the high-energy limit, Phys. Rev. D 57 (1998) 4069 [hep-ph/9711309] [InSPIRE].

[46] L. Tyburski, Reggeization of the fermion-fermion scattering amplitude in non-Abelian gauge theories, Phys. Rev. D 13 (1976) 1107 [inSPIRE].

[47] V.S. Fadin, E.A. Kuraev and L.N. Lipatov, On the Pomeranchuk singularity in asymptotically free theories, Phys. Lett. B 60 (1975) 50 [INSPIRE].

[48] L.N. Lipatov, Reggeization of the vector meson and the vacuum singularity in non-Abelian gauge theories, Sov. J. Nucl. Phys. 23 (1976) 338 [Yad. Fiz. 23 (1976) 642] [InSPIRE].

[49] E.A. Kuraev, L.N. Lipatov and V.S. Fadin, Multi-Reggeon processes in the Yang-Mills theory, Sov. Phys. JETP 44 (1976) 443 [Erratum ibid. 45 (1977) 199] [Zh. Eksp. Teor. Fiz. 71 (1976) 840] [INSPIRE]. 
[50] A.L. Mason, Radiation gauge calculation of high-energy scattering amplitudes, Nucl. Phys. B 120 (1977) 275 [inSPIRE].

[51] H. Cheng and C.Y. Lo, High-energy amplitudes of Yang-Mills theory in arbitrary perturbative orders. 1, Phys. Rev. D 15 (1977) 2959 [INSPIRE].

[52] E.A. Kuraev, L.N. Lipatov and V.S. Fadin, The Pomeranchuk singularity in non-abelian gauge theories, Sov. Phys. JETP 45 (1977) 199 [Zh. Eksp. Teor. Fiz. 72 (1977) 377] [INSPIRE].

[53] Z. Kunszt, A. Signer and Z. Trócsányi, One loop helicity amplitudes for all $2 \rightarrow 2$ processes in QCD and $N=1$ supersymmetric Yang-Mills theory, Nucl. Phys. B 411 (1994) 397 [hep-ph/9305239] [INSPIRE].

[54] V.S. Fadin, M.I. Kotsky and R. Fiore, Gluon Reggeization in QCD in the next-to-leading order, Phys. Lett. B 359 (1995) 181 [InSPIRE].

[55] V.S. Fadin, R. Fiore and M.I. Kotsky, Gluon Regge trajectory in the two loop approximation, Phys. Lett. B 387 (1996) 593 [hep-ph/9605357] [INSPIRE].

[56] V.S. Fadin, R. Fiore and A. Quartarolo, Reggeization of quark quark scattering amplitude in QCD, Phys. Rev. D 53 (1996) 2729 [hep-ph/9506432] [INSPIRE].

[57] J. Blumlein, V. Ravindran and W.L. van Neerven, On the gluon Regge trajectory in $O\left(\alpha_{S}^{2}\right)$, Phys. Rev. D 58 (1998) 091502 [hep-ph/9806357] [INSPIRE].

[58] G. Parisi, Summing large perturbative corrections in QCD, Phys. Lett. B 90 (1980) 295 [INSPIRE].

[59] G.F. Sterman, Summation of large corrections to short distance hadronic cross-sections, Nucl. Phys. B 281 (1987) 310 [inSPIRE].

[60] T.O. Eynck, E. Laenen and L. Magnea, Exponentiation of the Drell-Yan cross-section near partonic threshold in the DIS and MS-bar schemes, JHEP 06 (2003) 057 [hep-ph/0305179] [INSPIRE].

[61] V. Ahrens, T. Becher, M. Neubert and L.L. Yang, Origin of the large perturbative corrections to Higgs production at hadron colliders, Phys. Rev. D 79 (2009) 033013 [arXiv:0808.3008] [INSPIRE].

[62] A. Broggio, A. Ferroglia, B.D. Pecjak and Z. Zhang, NNLO hard functions in massless QCD, JHEP 12 (2014) 005 [arXiv: 1409.5294] [INSPIRE].

[63] V. Del Duca, C. Duhr and V.A. Smirnov, An analytic result for the two-loop hexagon Wilson loop in $N=4 S Y M$, JHEP 03 (2010) 099 [arXiv:0911.5332] [INSPIRE].

[64] L.J. Dixon, J.M. Drummond, C. Duhr and J. Pennington, The four-loop remainder function and multi-Regge behavior at NNLLA in planar $N=4$ super-Yang-Mills theory, JHEP 06 (2014) 116 [arXiv:1402.3300] [INSPIRE].

[65] V. Del Duca, L.J. Dixon, C. Duhr and J. Pennington, The BFKL equation, Mueller-Navelet jets and single-valued harmonic polylogarithms, JHEP 02 (2014) 086 [arXiv:1309.6647] [INSPIRE].

[66] J.R. Andersen and J.M. Smillie, Multiple jets at the LHC with high energy jets, JHEP 06 (2011) 010 [arXiv:1101.5394] [INSPIRE].

[67] S. Moch, J.A.M. Vermaseren and A. Vogt, The three loop splitting functions in QCD: the nonsinglet case, Nucl. Phys. B 688 (2004) 101 [hep-ph/0403192] [INSPIRE]. 